\title{
Centrosome integrity as a determinant of replication stress
}

\author{
Dissertation \\ for the award of the degree \\ "Doctor of Philosophy" \\ Division of Mathematics and Natural Sciences \\ of the Georg-August-Universität Göttingen \\ within the doctoral program Molecular Medicine \\ of the Georg-August University School of Science (GAUSS) \\ submitted by \\ Zainab Tayeh \\ from Kuwait city, Kuwait
}

Göttingen 2019 


\section{Thesis Committee}

Prof. Dr. Matthias Dobbelstein, Institute of Molecular Oncology, University Medical Center Göttingen (UMG)

Prof. Dr. Bernd Wollnik, Institute of Human Genetics, University Medical Center Göttingen, Göttingen (UMG)

Prof. Steven Johnsen, Department for general, visceral and pediatric surgery, University Medical Center Göttingen (UMG)

\section{Members of the Examination Board}

Prof. Dr. Matthias Dobbelstein, Institute of Molecular Oncology, University Medical Center Göttingen (UMG)

Prof. Dr. Bernd Wollnik, Institute of Human Genetics, University Medical Center Göttingen, Göttingen (UMG)

\section{Further members of the Examination Board}

Dr. Roland Dosch, Department of Developmental Biochemistry, University Medical Center Göttingen (UMG)

Prof. Dr. Heidi Hahn, Department of Human Genetics, University Medical Center Göttingen (UMG)

Dr. Nuno Raimundo, Department of Cellular Biochemistry, University Medical Center Göttingen (UMG)

Dr. Ufuk Günesdogan, Department of Developmental Biology, University Medical Center Göttingen (UMG)

Date of oral examination: 16.01 .2020 


\section{Affidavit}

Herewith I declare that the $\mathrm{PhD}$ Thesis entitled "Centrosome integrity as a determinant ofreplication stress" was written independently and with no other sources and aids than quoted.

\section{Zainab Tayeh}

Göttingen, November 2019 
This thesis is dedicated to family, Gökhan Güngor, and friends 


\section{List of figures}

\section{Introduction}

Figure 2.1: Centrosomes are multiprotein complexes that are replicated once per cell cycle. 3

Figure 2.2: Cell cycle vs. the centrosome duplication cycle.

Figure 2.3: Relation between centrosome amplification (CA) and its consequences in cancer 7

Figure 2.4: The hallmarks of cancer. 9

Figure 2.5: The role of centrosomes in development and disease..... 12

Figure 2.6: Consequences of replication-transcription conflict...... 15

\section{Results}

Figure 6.1: Depletion of centrosomal components interferes with DNA replication

Figure 6.2: Inhibition of the centrosomal component PLK4 interferes with DNA replication.

Figure 6.3: PLK4 inhibition leads to polyploidy after release.

Figure 6.4: Impairment of centrosomes causes replication stress independent of mitosis. .50

Figure 6.5: Centrosomal disintegration induces replication stress through p38 and MK2. .53

Figure 6.6: When centrosomes are disintegrated, MLK3 activates $p 38 / M K 2$ to reduce fork

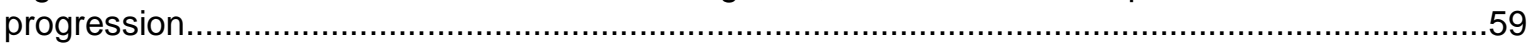

Figure 6.7: Replication stress upon centrosome disintegration requires RNA: DNA hybrids. 64 
Figure 6.8: PLK4 inhibition activates ATF2 and JUN to induce replication

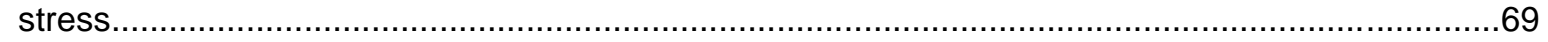

Figure 6.9: MK2 inhibition facilitates DNA replication and proliferation of cells derived from a Seckel patient.

Figure supplementary 1: MAP3K1 depleting prevents the activation of p38/MK2 in the H1299 cell line.....

\section{List of tables}

Table 1: Phosphoproteomics reveals a significant phosphorylation level of MAP3K1, CHK1 after Centrinone B treatment in $\mathrm{H} 1299$.

Table 2: Phosphoproteomics Shows the log2 of the most unregulated proteins upon Centrinone B treatment as in Table 1 


\section{Table of Contents}

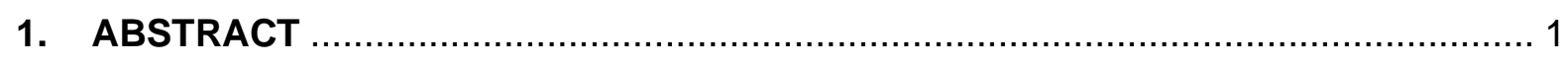

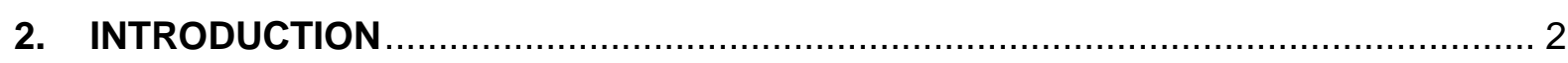

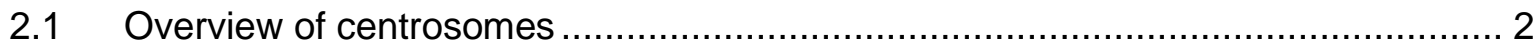

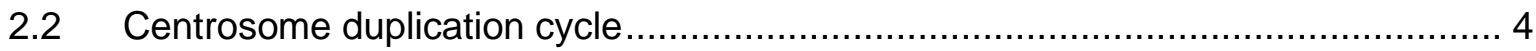

2.3 Centrosome in cancer and inherited diseases ................................................. 6

2.4 Chromosomal instability is a hallmark of cancer................................ 10

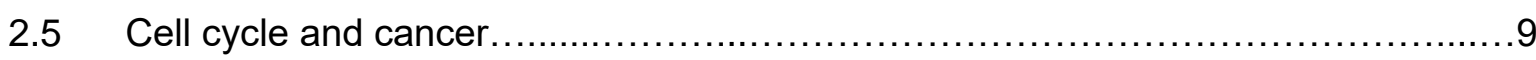

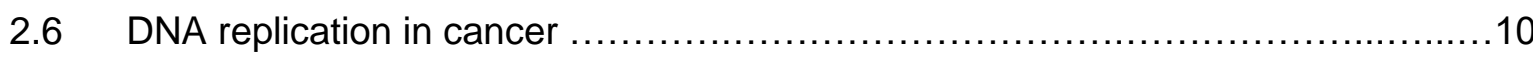

2.7 Seckel syndrome, a shared diseases between centrosomes abnormalities and ATR

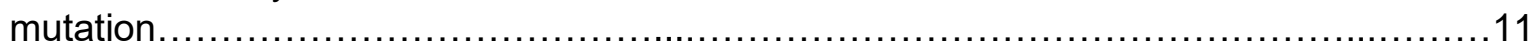

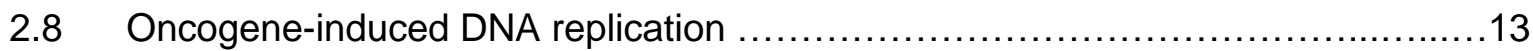

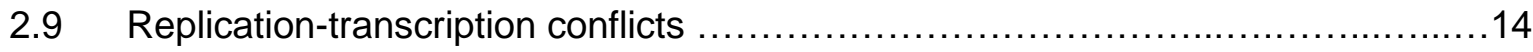

2.10 Crosstalk between centrosomes duplication and DNA replication...................16

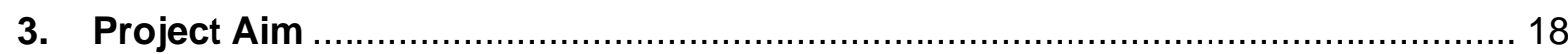

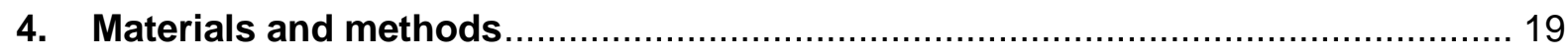

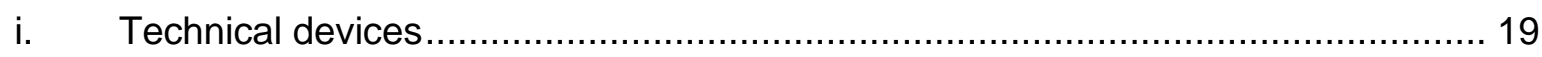

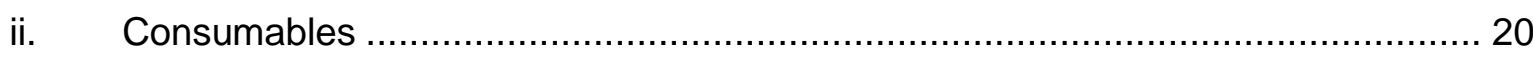

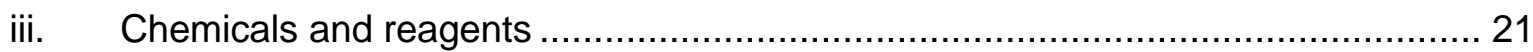

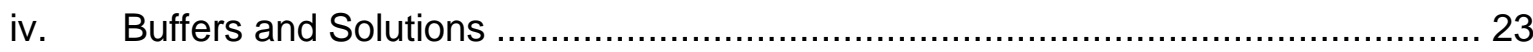

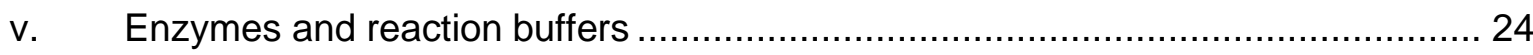

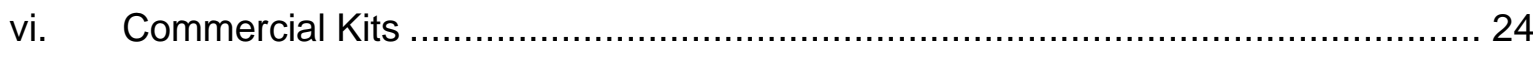

vii. Chemotherapeutics and Pharmacological inhibitors...................................... 25

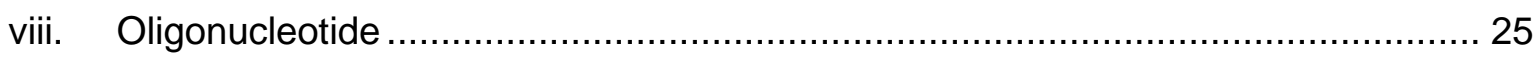

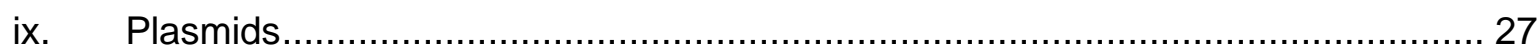

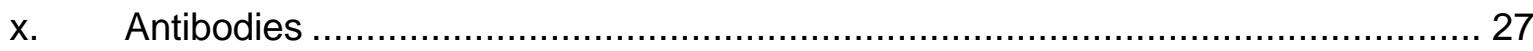

xi. Secondary Antibodies for Western Blot ....................................................... 29

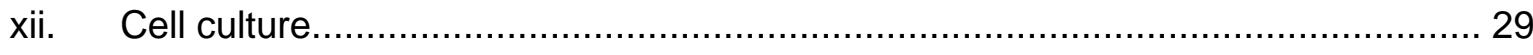

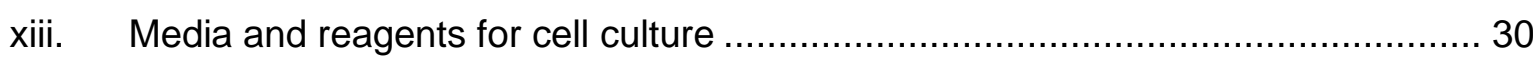

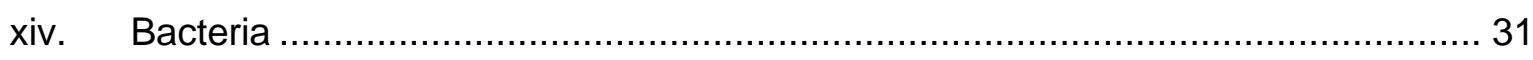

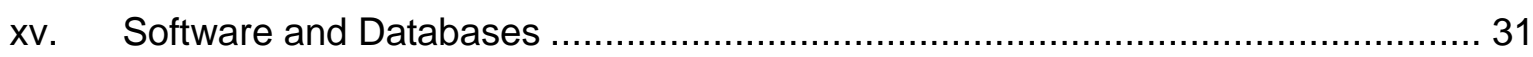




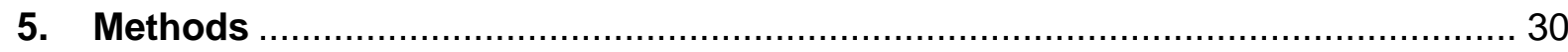

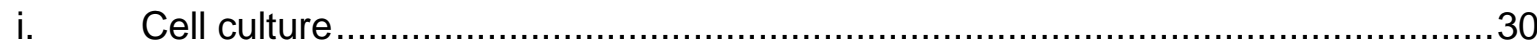

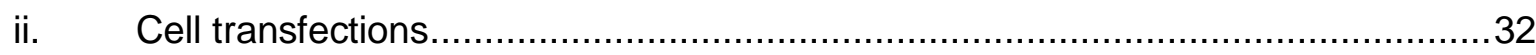

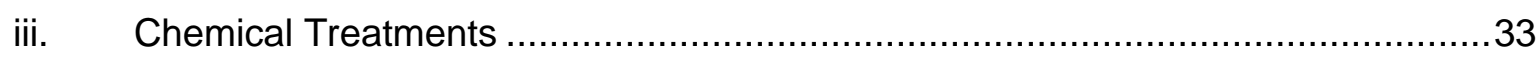

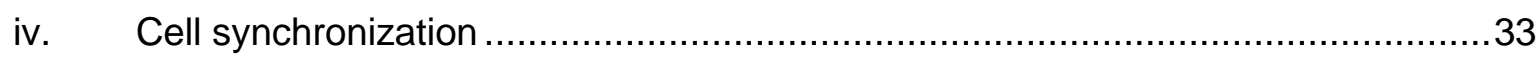

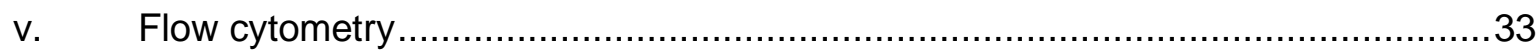

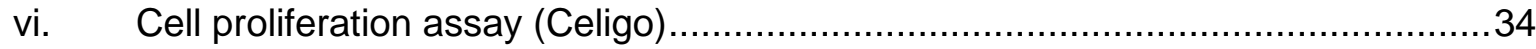

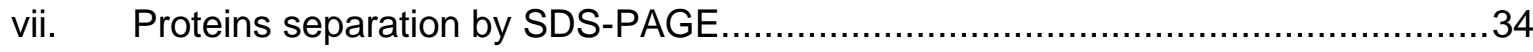

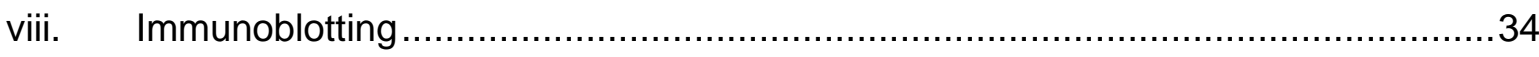

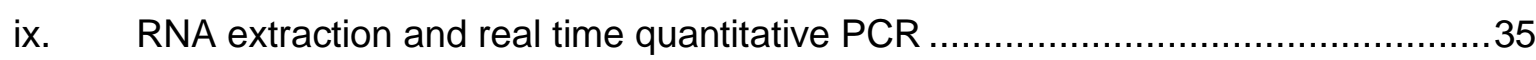

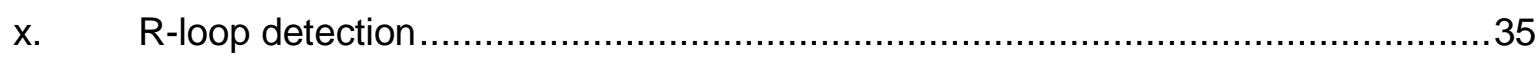

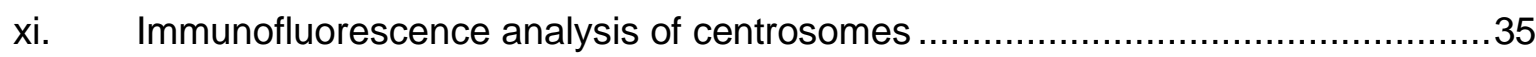

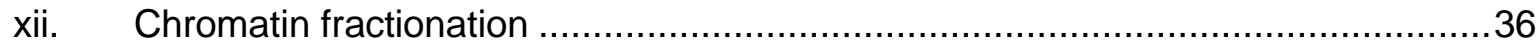

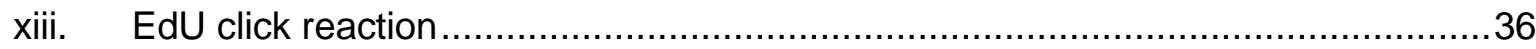

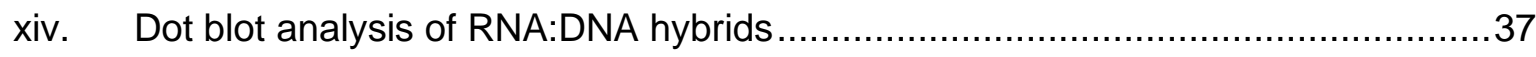

$\mathrm{xv}$. Chromosome spread analysis and chromosome counting ................................37

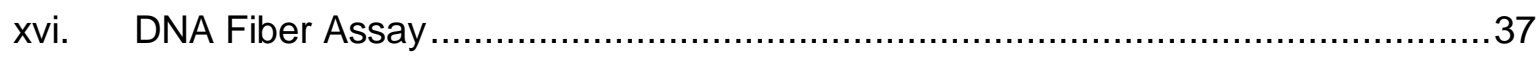

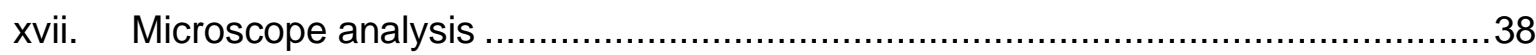

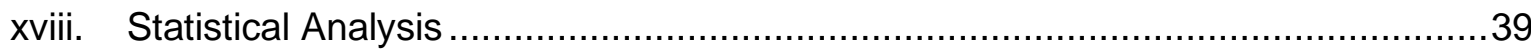

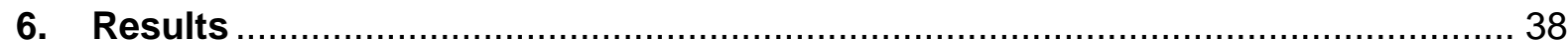

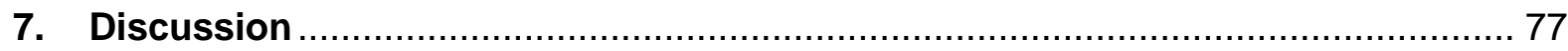

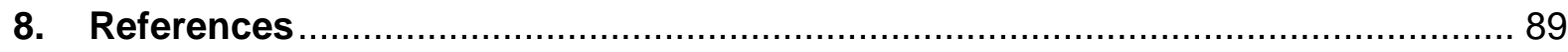

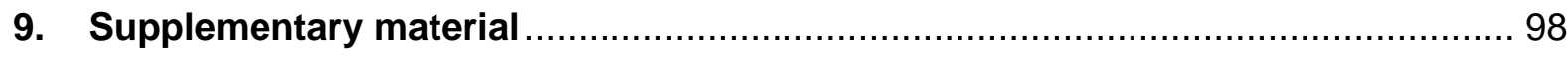

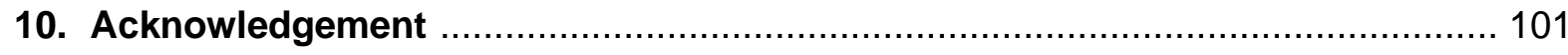

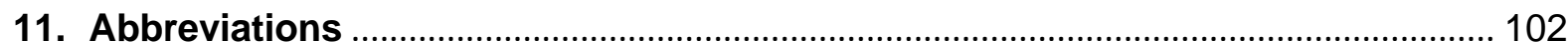

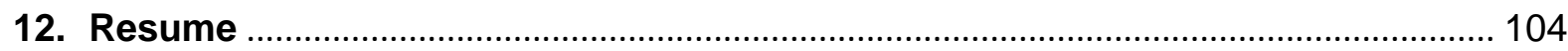




\section{ABSTRACT}

The centrosome functions as a microtubule-nucleating organelle for the mitotic spindle. Like the whole genome, centrosomes require accurate replication once per cell cycle. Here we show that the impairment of centrosome composition by depletion of centrosomal components or by the inhibition of centrosomal protein PLK4 reduces the progression of DNA replication forks in cancer cells. Importantly, the reduction in fork progression occurs even when the cells cycle are arrested at the G1 phase before damaging the centrosomes, excluding mitotic failure as the source of replication stress. Mechanistically, the kinase MLK3 associates with centrosomes. When the centrosome composition is impaired, MLK3 activates the kinases p38 as well as MK2/MAPKAPK2. RNA:DNA hybrids (R-loops) leading to DNA replication stress occur upon activation of the transcription factor JUN, which is a downstream target of p38. Finally, fibroblasts from Seckel syndrome patients harboring defective centrosomes showed replication stress, which was alleviated by inhibition of MK2. Similar replication stress has been observed upon deletion of the kinase ATR, and this genetic defect also causes Seckel syndrome.

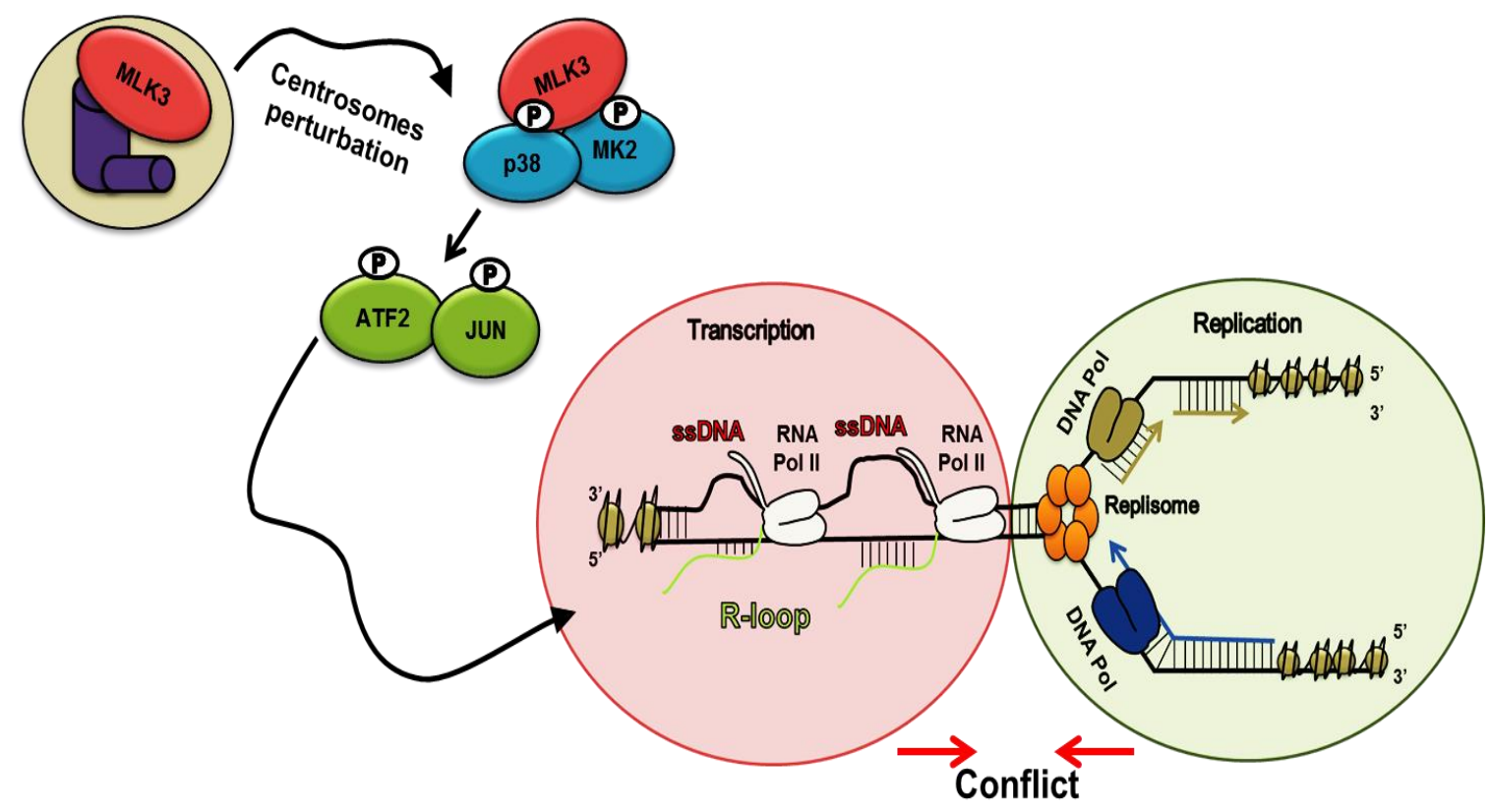

Keywords: polo-like kinase 4 (PLK4), Mitogen-Activated Protein Kinase 11 (MLK3), MAP kinaseactivated protein kinase 2 (MK2), Ataxia Telangiectasia And Rad3-Related Protein (ATR) 


\section{INTRODUCTION}

\subsection{Overview of Centrosomes}

Centrosomes are known to be the microtubule-organizing centers (MTOCs) of animal cells. They play their most prominent role during mitosis, in which they form two poles of the mitotic spindles. They associate with microtubules that, at their opposite ends, attach to chromosomes via kinetochores to separate the sister chromatids from each other $(1,2)$. Eduard Van Beneden discovered centrosomes in 1883, followed by their description in 1888 by the German biologist Theodor Boveri (3). Decades of studying centrosomes helped us to understand these organelles further, from revealing their exact structure to their duplication cycle to identify their multiple functions to conceal their contribution to human diseases. Nowadays, we know that centrosomes not only play a significant role in driving cell division but also are involved in the formation of cilia, cell adhesion, cell polarity, cell motility, and intracellular trafficking of proteins and organelles $(4,5)$.

Structural studies have revealed that each centrosome consists of two barrel-shaped centrioles (the mother and the daughter centriole), which are linked together in an orthogonal configuration. Both centrioles are highly alike in composition, yet they differ in the consistency of distal and sub-distal appended proteins, which mostly belong to the mother centriole and are mainly responsible for cilia generation and anchoring of microtubules (1). The mother centriole is responsible for nucleating and organizing microtubules, whereas the daughter centriole can only nucleate microtubules. Both centrioles are surrounded by a cloud of proteins called pericentriolar material (PCM) (Fig. 2.1). The PCM consists of several centrosomal proteins that have multiple functions such as centrosome duplication, maturation, and separation by the end of the cycle. Each centriole contains nine triplet microtubules arranged as a cylinder-like structure in a 9+3 pattern known as cartwheel structure (8). As centrosomes progress towards mitosis, additional proteins will be recruited to the site of centrosomes, e.g., PLK4, PLK1, and CDK2 (98).

Centrioles require permission to carry with their duplication cycle; such permission can be acquired merely after the cells pass the $M$ phase. Two steps govern the process of centriole duplication: the disengagement of centrioles by PLK1 and Separase at the G2-M phase, and the conversion of centriole-to-centrosome (4, 6, 103). After loosening the centriole engagement, centriole reduplication will be initiated, with the recruitment of the centrosomal 


\section{INTRODUCTION}

polo-like kinase (PLK4) by the scaffold protein CEP152 and CEP192 in early G1-phase. Once PLK4 is recruited to the site of centrosomes, it will start a cascade of centrosome proteins phosphorylation, which will be explained in detail in the next section. As the cell progresses to S/G2 phase, these newly formed procentrioles assemble their cartwheel and continue to elongate until they reach the length of the old mother-daughter centriole, for the reason that each "mother centriole" serves as a template to build up a new daughter centriole (6). Once the newly born centrioles are fully matured, the two centrosomes start to migrate apart from each other towards the cell poles, allowing chromosome segregation during the next cell cycle phase, which is called 'mitosis' (4) Although centrosomes are not obligatory for cell division,

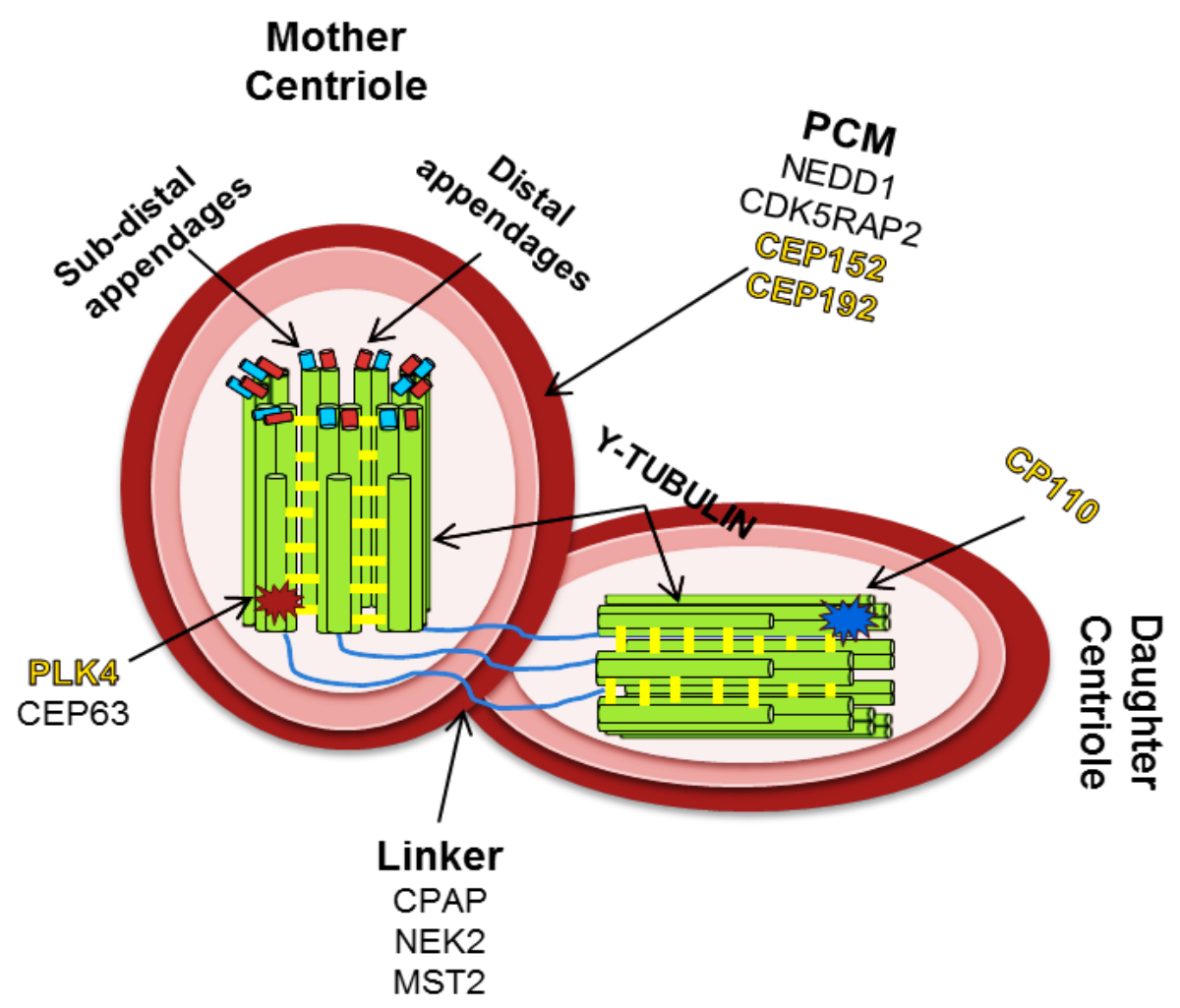

Figure 2.1: Centrosomes are multiprotein complexes that are replicated once per cell cycle detailed scheme of the fully matured centrosome. A nine-fold symmetry in microtubule triplets of the two centrioles (mother and daughter in green) and essential protein components of the pericentriolar material (PCM) that were identified to date are shown. Targets addressed in experiments of this study are highlighted in yellow (adapted from PIHAN, 2013). 


\subsection{Centrosome duplication cycle}

In dividing cells, centrosomes form bipolar spindles to organize chromosome segregation. Each daughter cell inherits one centrosome from the mother cell and thus needs to duplicate this centrosome once per cell cycle. Like DNA replication, centrosome duplication is a crucial process within the cell, and therefore it is highly regulated by cell cycle-coupled processes that are essential to ensure the only one-time duplication per cell cycle. Once the cell is at the G1/S transition, each pre-existing centriole will start forming their pro-centriole, which will remain in a close distance to their parent's centrioles till late of G2 (9-12).

Licensing centriole duplication is not an exclusive process for centrosomes based proteins; like PLK1 and Separase $(103,118)$, but also cell cycle several regulators are involved in monitoring and controlling the faith of the centrosome duplication process; for example, the centrosome cycle is mediated by cyclin-dependent kinase 2 (CDK2) (98), cyclin A and cyclin $E$ (9). Several studies proposed that CDK2 has many substrates that are responsible for the regulation of centrosome duplication. These substrates mainly include Centriolar Coiled-Coil Protein 110 CCP110, and Serine/threonine-protein kinase MPS1 (MPS1) (Figure 2.2) $(9,98)$.

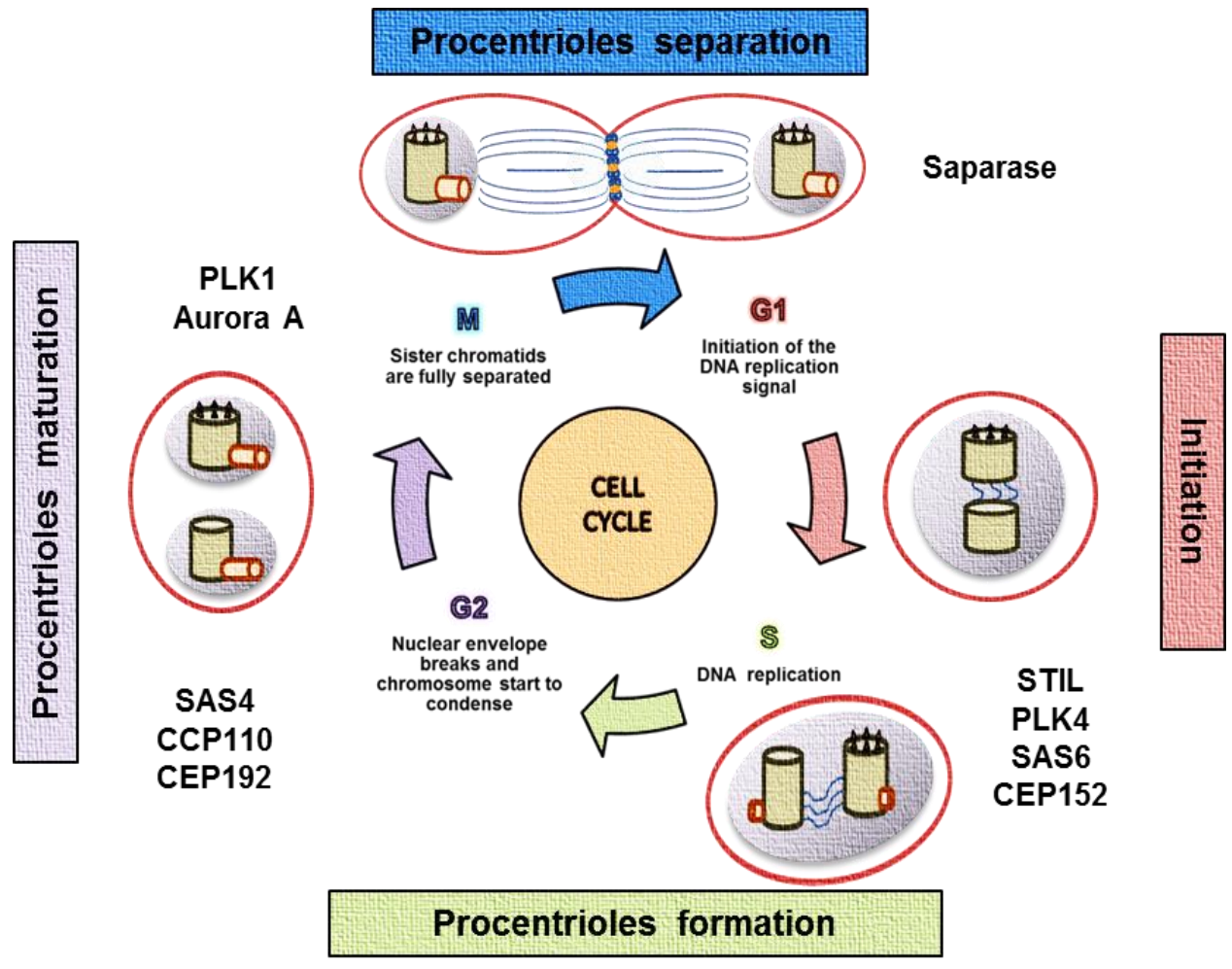

Figure 2.2: Cell cycle vs. the centrosome duplication cycle. Both cycles are being regulated and controlled by several regulators and factors to ensure a faithful cell division. 
Initially, the centrosomal proteins CEP152 and CEP192 recruit polo-like kinase 4 (PLK4) and locate it on the mother centriole, where the new centriole will be built $(10,11)$. The recruited PLK4 will then bind to centrosomal protein STIL, and upon this binding, PLK4 will experience conformational changes, which will lead to its activation (10,11). Activation of PLK4 then will then phosphorylate STIL, triggering the recruitment of spindle assembly abnormal protein 6 (SAS6) to form the "core module for centriole duplication" (12).

While STIL is targeted by phosphorylation of PLK4 $(12,13)$, SAS- 6 self-assembles into a cartwheel, forming the basis of the central tube for nucleation of microtubule triplets in a nine-fold symmetry $(14,15)$. Despite the lack of knowledge in the downstream events of centriole duplication, yet there are some clues that CEP135 helps to connect SAS-6 to the outer microtubules of the microtubule triplets $(15,16)$. As cells progress through S-phase and towards G2-phase, daughter centrioles on both mother centrioles need to be elongated. The length of newly born centrioles is controlled by the centrosomal proteins CP110 and CPAP (16). In parallel to the elongation, many different proteins are assembled in high order into a growing PCM.

The PCM protein pericentrin (PCNT) plays a central role in organizing the assembly, which is essential to provide mature centrosomes with the capacity to nucleate many microtubules during mitosis (17). When both centrosomes are fully assembled, they break apart at the G2/M-transition (disengagement) with the help of PLK1 and Separase $(103,118)$, and each centrosome moves towards one side of the condensed chromosomes - followed by the microtubules nucleation by $\mathrm{Y}$-tubulin ring complexes ( $\mathrm{Y}$-TuRC), which later on will serve as spindles during mitosis to pull apart the sister chromatids. After cytokinesis, typically one centrosome with former mother and daughter centriole is now loosely attached by a flexible linker which remains in each daughter cell, and the cycle can restart once again (Figure 2.2) $(4,6)$. 


\subsection{Centrosome in cancer and inherited diseases}

A century ago, the German biologist Theodor Boveri hypothesized that increased centrosome numbers could drive tumorigenesis (3). This hypothesis is currently supported by evidence demonstrating that centrosome amplification (CA) is found in precursor lesions and could initiate events in carcinogenesis $(18,19)$. Additionally, CA is a well-known hallmark in around $10 \%$ of all human cancers $(20,21)$. Without a doubt, CA is existent in a wide range of both hematopoietic and solid cancer (Figure 2.3), these defects have been noticed in early and advance cancer development, and they were associated with poor clinical outcome and progressive tumor grade (30).

In many cases, CA can raise by two mechanisms; one is by centriole over-duplication and the second by cell doubling events (e.g., cytokinesis failure or cell-cell fusion). As a result of $\mathrm{CA}$, cells with supernumerary centrosomes generate genetic diversity through asymmetric cell divisions on abnormal spindles with chromosome miss-aggregation $(22,23)$. Correspondingly, CA highly correlates with aneuploidy and chromosomal instability in cancer, leading to enhance cancer invasiveness through the disruption of cell-cell contacts and altering the position and size of cell-cell junctions, through increasing the activity of Rac1 and Rho GTPases $(21,24)$.

PLK4 is one of the master regulators of centriole duplication, which, when overexpressed, can induce CA, among others, through the generation of multiple procentrioles $(25,26)$. Overexpression of SAS6 (12), STIL (27), and pericentrin (28) can also result in overduplication of centrioles, which they might contribute to tumorigenesis yet need to be further explored. The overexpression of several centrosome components enhances cancer migration (29). PLK4 is known to be overexpressed in breast, lung, and colorectal cancer and correlates with not only worse outcomes but also predicts resistance to chemotherapeutic drugs (Figure 2.3) (30). All of this makes targeting centrosome components known to play a part in centrosomes amplification like PLK4 is increasingly becoming a potential target for cancer treatment. 

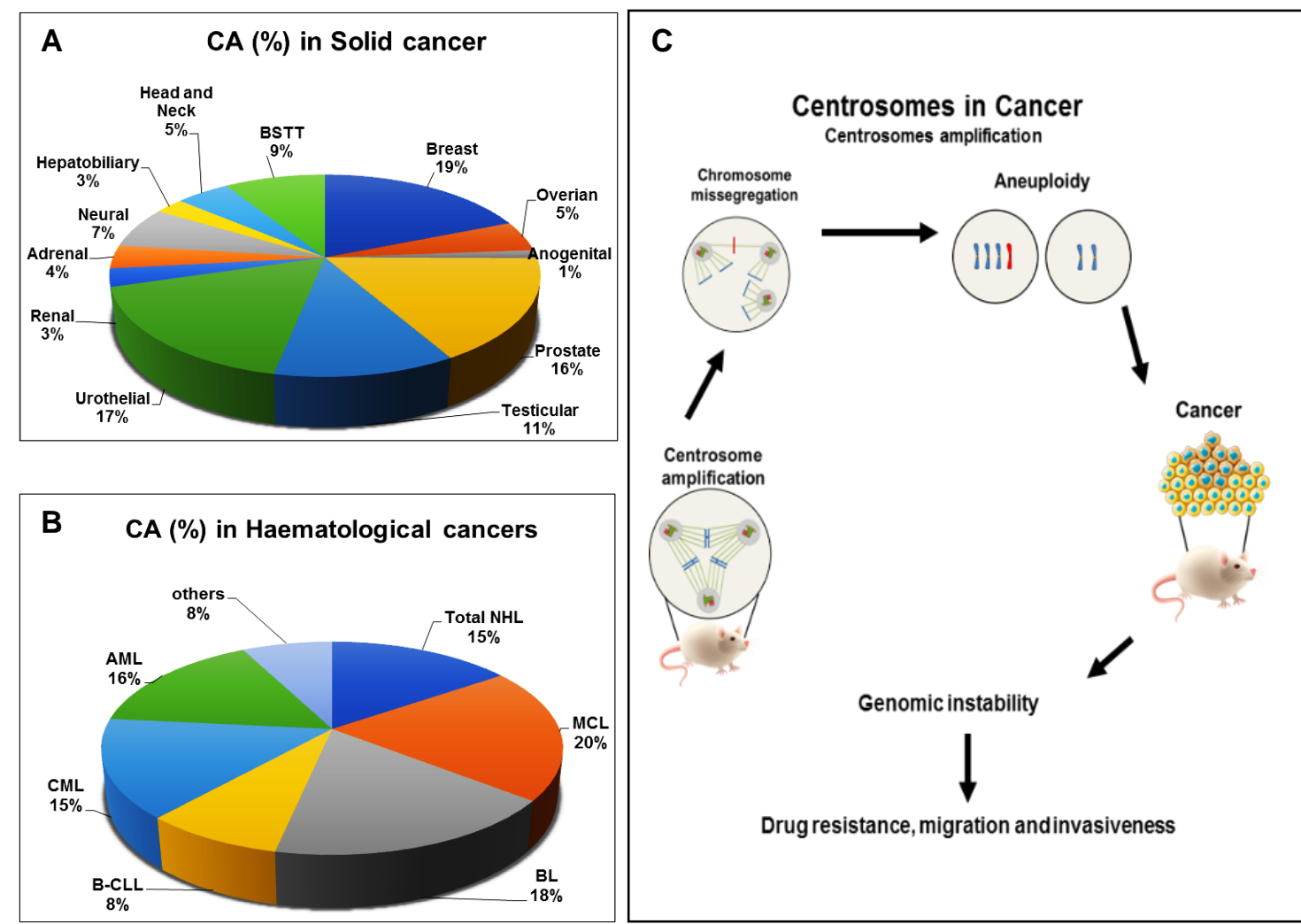

Figure 2.3: Relation between centrosome amplification (CA) and its consequences in cancer.

(A-B) Centrosome abnormities are connected to several kinds of cancer: Solid cancer as well as hematological cancer. The percentage represents the CA-percentage within each cancer species. The figures are modified from (Chan, J.Y, 2011) (20). (C) One of the hallmarks of cancer is centrosome amplification. The obtained extra copy of centrosomes can control the fate of the cell and change the type of cancer behavior (111).

Centrosome abnormalities are not only connected to cancer. Cells with centrosomal defects suffer from a defective cell division, which is found to be especially crucial during neural development. Therefore, unbalanced centrosome numbers were postulated to cause cell death leading to intrauterine growth retardation, which could explain microcephaly (73). For instance, a genetically heterogeneous neurodevelopmental disorder known as autosomal primary recessive microcephaly $(\mathrm{MCPH})$ is caused by mutations in at least nine centrosomal genes such as; CEP152, CDK5RAP2, ASPM, CPAP, and STIL. MCPH is characterized by reduction in head circumference at birth and non-progressive mental retardation, which primarily affecting the size of the cerebral cortex; if the MCPH is associated with dwarfism, the syndrome is called microcephaly primordial dwarfism (PD). PD defines as group of autosomal recessive human genetic disorders includes; Seckel syndrome, microcephalic osteodysplastic, Meier-Gorlin syndrome and PD (MOPD) types I and II are the most prominent 
diseases of this group. PD paitents suffer from pre- and postnatal growth failure accompanied by microcephaly (31-34). Interestingly, such syndromes can emerge from mutations in centrosomal components, including PLK4, CEP152, PCNT, and many others, or ATR/ATRIP (linked to replicative stress). Although not much evidence was found on higher cancer prevalence in patients with Seckel syndrome, patient cells show chromosomal instabilities, a hallmark of many cancer cells (31-34).

\subsection{Chromosomal instability is a hallmark of cancer}

Chromosomal instability (CIN) is a hallmark of many human diseases like cancer, neurodegenerative diseases, and age-related diseases. CIN, as shown in many recent publications, can cause structural and numerical chromosome abnormalities (69, 70). As oncogene-induced replication stress or poor replication fork maintenance is an essential driver of genomic instability, also centrosomes abnormalities such as having excessive centrosomes, caused by hyper amplification can induce CIN is when the (31) (Figure 2.4).

The mechanisms behind the induction of centriole amplification are not fully described yet, but since it is found in many cancers at early stages, targeting this process would represent an attractive drug approach (20). Multiple centrosomes would propose multipolar mitotic divisions leading to severe aneuploidy and cell death in normal cell conditions. Unfortunately, this is not the case in cancer because cancer cells have found a way to overcome aneuploidy. One way in which cancer cells can avoid lethal aneuploidy is by clustering their centrosomes; a mechanism was first described by (Ring et al. 1982), in which cancer cells ensure bipolar spindle formation during mitosis despite multiple centrosomes, and thus cancer cells rely on this process for survival. Interestingly, defects in the clustering of amplified centrosomes were found to cause apoptosis and microcephaly in murine neural stem cells (73). 


\section{INTRODUCTION}

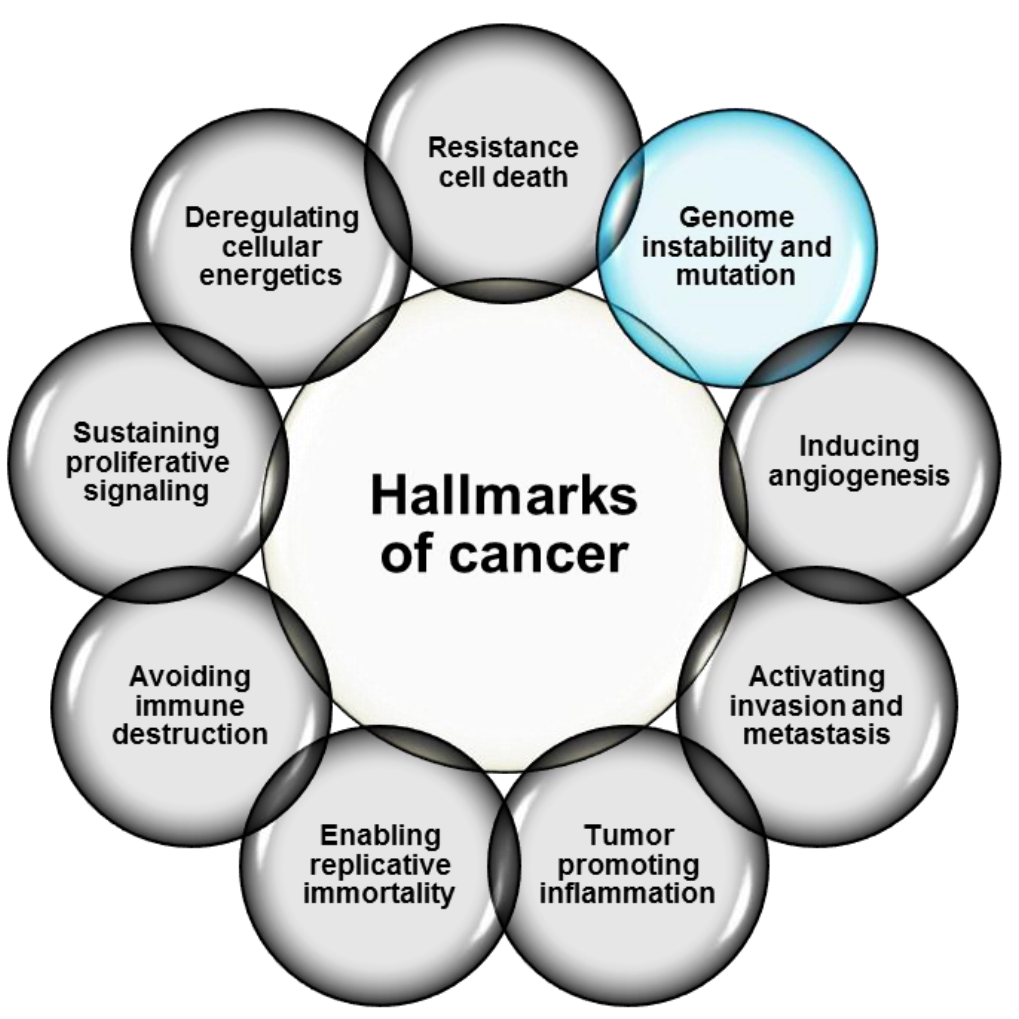

Figure 2.4: The hallmarks of cancer. Modified schematic representation of the hallmarks of cancer adapted from Hanahan et al., 2011. Chromosomal abnormalities leading to genomic instability and increasing the mutation rate in the cell is one of the main hallmarks of cancer.

In summary, cancer cells with clustered centrosomes were found to result in higher rates of CIN, partly because of mitotic kinetochore attachments and lagging anaphase chromosomes, which could promote tumorigenesis (31).

\subsection{Cell cycle and cancer}

Actively dividing cells go through a series of events that are collectively known as the cell cycle. As a survival mechanism, cells need to replicate their genetic material during this cycle. However, cell cycle alternation is found in several kinds of diseases, and for such reason, the cell cycle is considered to be one of the most critical processes within our body. In healthy cells, the cell cycle is controlled by several checkpoints, and thereby the process is highly regulated and precise. However, in cancer cells, this is not the case (35). 
Several proteins and checkpoints are required to ensure a faithful cell division and to overcome the obstacles that dividing cells may face during the cell cycle (35). The primary purpose of several checkpoints within the cell cycle is to guarantee that damaged or incomplete DNA is not being passed on to the newly born daughter cells. There are three main checkpoints within the cell: G1/S, G2/M, and the spindle assembly checkpoint (35).

The cell uses the G1/S checkpoint to make sure that it has enough raw materials to replicate its DNA fully. G2/M is another checkpoint which guarantees that the cell has enough cytoplasmic phospholipids for the newly born cells. Besides, cells check for unreplicated or damaged DNA after the S-phase and before they undergo mitosis (Figure 2.2) (36). Finally, the mitotic checkpoint is responsible for checking the spindle that has been formed and attached to the condensed chromosomes, and that all chromosomes are aligned before the onset of anaphase $(35,36)$.

\subsection{DNA replication in cancer}

In dividing cells, beside centrosome duplication also DNA replication is required in every cell cycle. DNA replication must reach maturity before the onset of mitosis to ensure that upon cytokinesis, each daughter cell inherits a complete set of genes $(37,38)$. Replication is a highly regulated process, and any condition that compromises it is referred to as replication stress. Replication stress can arise as a result of several factors. Generally speaking, it can occur due to DNA lesions, diminished expression of replication factors, or a shortage in nucleotides. Also, replicative stress can be caused due to the expression of several oncogenes, for instance, RAS, HPV E6/E7, and cyclin E.

Both faithful chromosome segregation during M-phase and accurate DNA replication during S-phase are the two main requirements for the cell to maintain genome integrity and avoid genome instability (39). Therefore, the process of replication is a prerequisite of life that is tightly regulated (40). For that reason, several mechanisms have evolved to ensure errorfree copying, to repair potential mistakes, to stop cell division, and to drive faulty replicated cells into apoptosis (41). Some of which these mechanisms are; the contribution of the DNA polymerase in nucleotide selection and proofreading, mismatch repair mechanism, which comes to correct what the proofreading mechanism failed to fix and managing the balanced supply of nucleotides (41). As DNA replication is a very delicate process, if it is disturbed by replication errors, it would cause a delay in the S-phase and induce replicative stress (38). 
Replication stress is not a common feature of healthy cells but is a significant cause of genome instability and linked to aberrant and tumor cells. Several mechanisms can explain the occurrence of this phenomenon in various kinds of cancers. Initially, replicative stress is induced after the activation of several oncogenes, which alter the replication timing and progression $(38,42)$. One more reason beyond the initiation of replicative stress in cancer is when the DNA damage checkpoint is not able to recognize the abnormalities on the DNA fork anymore and fails to protect the stalled forks and to restart replication progression through the activation of dormant replication origins (42). Together with dysfunctions of the DNA damage checkpoint, replicative stress can also be induced by dysfunction in DNA repair mechanisms, which upon DNA breaks are needed to repair the damage and prevent the fork from being stalled $(38,42)$. Reactive oxygen species (ROS) or misincorporated nucleotides are also sources of stressors that cause DNA lesions and can induce replicative stress (43). If the replication fork cannot continue proceeding along the DNA strand due to damage-induced obstacles, it stalls, whereas helicases usually progress to unwind the DNA helix and thereby to expose large areas of single-stranded DNA (ssDNA) (44). A stalling DNA replication fork activates the DNA damage response (DDR), a complex signaling cascade involving central mediators such as the kinases ATR and ATM, as well as their targets, the checkpoint kinases CHK1 and CHK2. Upon DNA damage, ATR is first recruited to the site of damage by $f$ replication protein $A(R P A)$, which covers ssDNA, following phosphorylation of its targets, i.e., CHK1 and Histone 2A.X. Subsequently, DDR signaling can mediate cell cycle arrest so that DNA replication errors and lesions will be repaired. When DNA damage becomes so severe that double-strand breaks occur, ATM-CHK2-signaling is activated, often leading to cell death to protect the whole organism from accumulating DNA errors (45). Cancer cells often display high levels of replicative stress and genomic instability because their accumulated mutations promote proliferation despite the lack of checkpoints and DNA repair systems (38).

\subsection{Seckel syndrome, a shared disease between centrosome abnormalities and ATR mutation}

Besides cancer, Seckel syndrome is a well-known genetic condition characterized by the accumulation of replicative stress through a mutation in ATR or its interacting protein ATRIP. While the loss of function mutations of ATR/ATRIP were found to be embryonically lethal (46), hypomorphic mutations resulting in reduced protein levels clinically caused by Seckel syndrome $(47,48)$. Seckel syndrome can be mimicked by a genetic mouse model harboring a comparable ATR mutation and showing signs of Seckel syndrome (49). Seckel syndrome (SCKL/SCKS) is a rare genetic disorder yet well-characterized disease that belongs to the group of primary autosomal recessive microcephaly (MCPH) and Seckel syndrome 
spectrum disorders (50). Patients of Seckel syndrome suffer from primordial dwarfism, microcephaly, a sloping forehead, and beaked nose, which is why the condition is also called "bird-headed dwarfism." In some cases, patients are mentally disabled due to their underdeveloped brain, and their body is of short stature, while most organs developed to normal function $(32,50)$.

What is more intriguing about this disease is that mutations cause the majority of clinical Seckel syndrome cases in ATR/ATRIP, but also in the centrosomal protein 152 (CEP152; SCKL5), identified by Kalay, E. et al. (Figure 2.5) (32). In centrosomes, Cep152 is a scaffold protein localized in the PCM. Besides its functional involvement in centriole duplication, where it is responsible for the recruitment of PLK4 to the centrosome site, CEP152 was identified as a "genome maintenance protein." One of the studies that looked at patient fibroblasts harboring a homozygous splice donor-site mutation revealed that loss of CEP152 protein function caused centrosomal and mitotic aberrations. Most strikingly, the authors also observed increased phosphorylation of histone $2 \mathrm{~A}(\mathrm{Y}-\mathrm{H} 2 \mathrm{~A} . \mathrm{X})$, which is a marker for replicative stress induced by ATR-dependent DDR, in CEP152 Seckel fibroblasts, proposing a function of CEP152 in protecting genomic integrity (32). Mutated CEP152 has also been identified in primary autosomal-recessive microcephaly (MCPH9; 51), characterized by small brain size and mental retardation, but standard height. Other types of Seckel syndrome and primary microcephalia have also been attributed to mutated centrosomal components: Cep152interacting protein Cep63 (SCKL6; 52), Microcephalin 1 (MCPH1), CEP135 (MCPH8), CDK5RAP2 (MCPH3), CPAP/CENPJ (MCPH6/ SCKL4) and others (50). Besides, more proteins that are essential for the duplication of centrosomes were found to be mutated in different types of microcephaly: Spindle assembly abnormal protein 6 (SASS6; MCPH14) and Polo-like kinase 4 (PLK4) (53). Additionally, mutations in the centrosomal pericentrin (PCNT) cause Seckel syndrome (54) as well as microcephalic osteodysplastic primordial dwarfism (MOPD2; 55), which displays small brain size but less severe mental retardation.
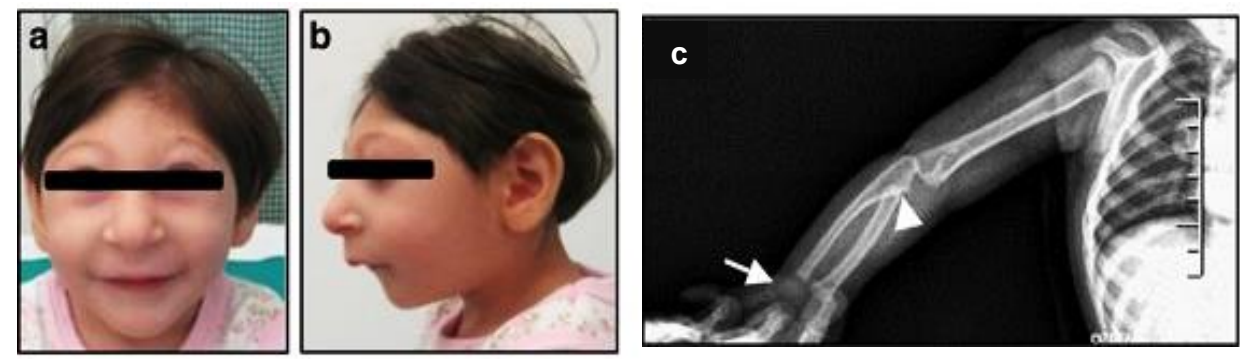

Figure 2.5: The role of centrosomes in development and disease. The phenotype of a PLK4-Seckel patient (a-b) Frontal and lateral view of a PLK4-Seckel patient. (c) Picture of the right arm showing hypoplasia (arrow head) (112). 
Thus, both the loss of centrosomal proteins and a mutation in proteins related to DDR result in similar developmental defects.

\subsection{Oncogene-induced DNA replication}

DNA replication must be tightly regulated during each cell cycle (41). Our genome is facing many intrinsic and extrinsic risk factors that interfere with DNA replication processes (45). One of these factors is the activation of one or more oncogene. Oncogenic activation is affecting DNA replication in which the DNA replication fork progression will be halted by either slowing it down or stalling it during the S-phase, leading the fork to collapse into DNA double-strand breaks, as well as incomplete sister chromatid separation (56). Oncogenic activation and DNA replicative stress promote chromosomal instability, which is a marker of tumor cells (56).

The activation of one of these oncogenes such as RAS, CDC25A, MYC, or CYCLIN E is sufficient to induce DNA replicative stress $(57,58)$. The oncogenes, as mentioned above, are considered as growth factors that stimulate cell proliferation. Initially, it was shown that the activation of CYCLIN E is responsible for increased cell proliferation by accelerating the entry to S-phase, leading to an increase in DNA replication initiation, which eventually causes replicative stress $(57,58)$. However, increasing cell proliferation by shortening the G1 phase is not the only mechanism of oncogene inducing replicative stress. The activation of oncogenes can activate the cell cycle also through the activation of transcription processes (59). The activation of transcription is mainly caused by the activation of RAS and MYC oncogenes (59). The activation of RAS oncogene stimulates the transcription through the extracellular-signal-regulated kinases (ERK), which in turn activates several transcription factors such as TBP, TIFIIIB, UBTF, and TIFIA (59). In contrast, the activation of the other oncogene MYC promotes transcription by activating the three RNA polymerases (59). However, the legitimate question at the moment is: How does the activation of transcription lead to DNA replicative stress?

Previously, it was shown that the activation of the transcription machinery has the potential to cause replication stress and fork collapse as well as genomic instability, which can be due to two reasons. Firstly, the mechanisms of transcription and replication are competing over the same DNA template in a similar affinity so that the active proteins may directly collide. Secondly, the formation of what is known as RNA: DNA hybrids (R-Loops) is another crucial reason for the collision between the DNA replication machinery and transcription (59). 


\subsection{Replication-transcription conflicts}

Both transcription and replication occur at a high frequency within cells and, therefore, are required to be completed with high fidelity to conserve genetic information and cell functionality (60). As described in sections 2.5 and 2.6, our DNA is packed into chromatin, meaning it needs to be unwinded and separated into two DNA single strands before being replicated or transcribed. Both polymerases, DNA and RNA, need to act in coordination with several enzymes and factors, such as helicases to open up the double-stranded DNA and topoisomerases that are required to solve the DNA topology $(61,62)$. The DNA replication machinery is assembled at precise genomic locations, called the origin of replication, while the transcription complex is assembled on areas called promoters. Both can move for many $\mathrm{kb}$ and polymerize in the direction of 5'-3' before the termination occurs. During DNA synthesis, one replisome replicating the leading strand while the other is replicating the lagging strand. While, during transcription, several RNA polymerases transcribe one strand of the opened DNA while the other strand remains as ssDNA. Therefore, the competition over the same DNA template increases the chance of both processes to interfere with each other (Figure 2.6) (61, $62)$.

A conflict that might arise due to the interference between DNA replication and transcription can lead to DNA damage and, eventually, genomic instability. The main reason behind such a conflict is the fact that these two abundant mechanisms are sharing the same DNA template. Unscheduled replication-transcription conflicts change the gene transcription program and generate replication stress, reducing fork speed (62).

On the other hand, cells have evolved numerous processes to reduce such conflicts and to rescue any replication fork that suffers from damage during conflicts for example Auxiliary Helicases, which their activities may assist the replisome dislodge transcription complexes ahead of the replication fork, in addition the S-phase checkpoint controls and responds to replication forks stalled at transcription complexes, by either controlling the tRNA gene transcription which reduces the interference with replication or by the activation of Mec1/ATR kinase followed by the phosphorylation of the nucleoporin Mlp1 which promotes fork progression by lowering the topological tension (136). However, the main question is: How can these two processes cope together, and how are conflicts between them being regulated?

During transcription and replication, chromatin structure is being disrupted to allow the transcription and replication machineries to initiate. Firstly, this occurs by unwinding the double 


\section{INTRODUCTION}

strands DNA (63). As transcription-replication machineries share the same DNA template, they might meet head-on (65), which may lead to a collision between them. One main pathological result of replication-transcription collisions is the formation of stable RNA:DNA hybrids known as R-loops, which also displace a naked ssDNA (60).

An extended RNA:DNA hybrid model suggests that the RNA:DNA hybrids could be the result of an extension of eight-base pair (bp) RNA:DNA hybrid (66) within the transcription bubble as RNA-Polymerase II (Pol II) elongates. R-loop structurs are mostly generated by Pol II, transcribing a C-rich DNA template so that a G-rich transcript is produced. R-loops generation within the cells depends mainly on three features: high density of guanine, negative supercoiling, and DNA nicks (66).

R-loop formation can lead to a slowing or collapse of the replication fork leading to genomic instability $(67,68)$. The resolution of this RNA: DNA hybrids depends on specific endonucleases known as RNases $\mathrm{H}$, which cleave the RNA strand of RNA: DNA hybrids (6668).

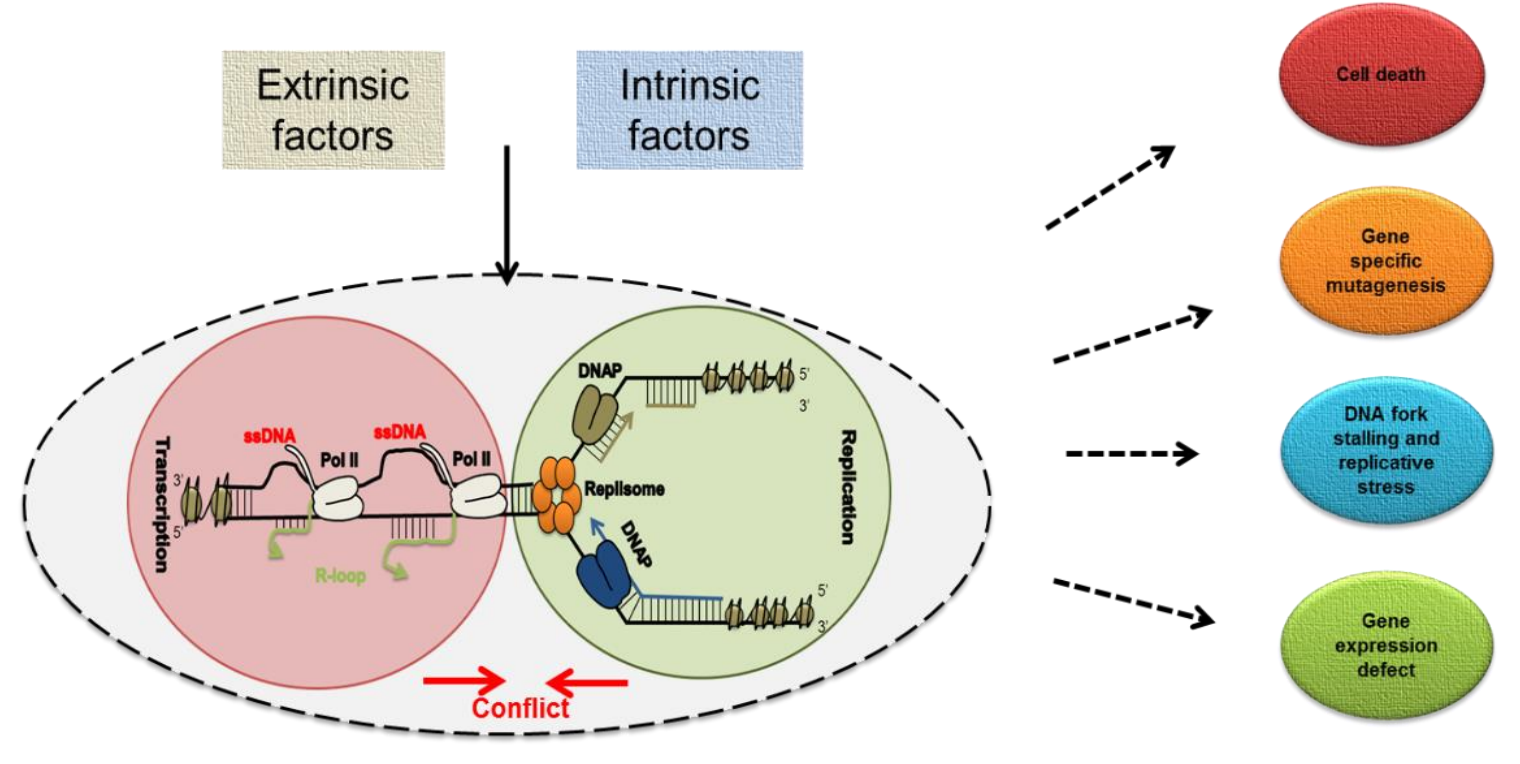

Figure 2.6: Consequences of replication-transcription conflict. Schematic representation of head-on collision between replication and transcription machinery. Raised conflict will lead to pervasive R-loop formation.

\subsection{Crosstalk between centrosome duplication and DNA replication}

Both processes of DNA replication and centrosome duplication need to be accurate. Therefore, to achieve this reliably, both methods must integrate signals which become even 
more relevant under stressed conditions. Consequently, we propose that replicative stress influences centrosomal components, and inversely the integrity of centrosomes promotes successful DNA replication.

Accumulating evidence suggests that proteins of the DDR signaling, induced upon replicative stress, are involved in centrosomal processes. The checkpoint kinase CHK1 was shown to mediate centrosome amplification after ionizing radiation of human cells $(74,75)$. CHK1 was postulated to shuttle between centrosomes and nuclear localization to sites of DNA damage and to be partially activated at the centrosome. More in-depth analysis revealed that DNA damage caused an expansion of PCM through the activity of CHK1 in the nucleus is due to control the DNA damage response (DDRs), which was supported by the PCM-component pericentrin (PCNT), and diminished through microcephalin (MCPH) (76).

For PCNT mutations in Seckel syndrome, it was found that ATR-dependent signaling upon DNA damage, mediated through $\mathrm{CHK} 1$, is defective in patient cells, which display similar characteristics to ATR-mutated Seckel cells (54). Thus, CHK1-ATR signaling seems to play an essential role in connecting the DDR and centrosome integrity.

Related kinases, CHK2 and MAPK-activated protein kinase 2 (MK2), partially localize to the centrosome as well, and CHK2 has even been found to be directly phosphorylated by PLK4 at centrosomes (77). Additionally, the tumor suppressor p53, which we have elucidated to support the progression of DNA replication forks (78), is partially localized to centrosomes and is proposed to regulate centriole duplication (79).

Intriguingly, loss, as well as amplification of centrosomes, triggered a p53-response (73), whereas p53 depletion caused centriole over-duplication (80). Therefore, several proteins have been identified with dual localization at centrosomes and on DNA. Indeed, it's also intriguing to investigate and fully discover those proteins which have potential crosstalk between DNA replication and centrosome assembly.

Centrosome abnormalities and replication stress are commonly observed features of cancer cells and critical drivers of genomic instability giving rise to human diseases, such as cancer or developmental and inherited diseases. Replication stress induces several signaling cascades that enhance ATR and CHK1 activity, which in turn enhances the processivity of replication forks despite all stressors in the surrounding environment (46-49). In addition, a hypomorphic allele of ATR and its partner ATRIP can give rise to a rare yet well-defined syndrome called Seckel syndrome 9 (SCKL9), but this is only one type of this syndrome. 


\section{INTRODUCTION}

SCKL4, 5, 6 are caused by a mutation in some of the centrosomal components such as CENPJ, CEP152, and CEP63, respectively (46-49). Altogether, this might suggest that DNA replication and the integrity of centrosomes might feed into a similar signaling pathway. 


\section{PROJECT AIM}

Our main aim in this project is to understand how the integrity of centrosomes can affect DNA replication, to further understand the interdependence of replication stress and centrosomal integrity during S-phase. We want to reveal whether there is any potential crosstalk between the two main types of duplication during the cell cycle: DNA replication and centrosomes duplication. Furthermore, we are interested in clarifying the leading players which are responsible for such an effect.

How do deficiencies in centrosomal composition lead to replicative stress? This central question can be further subdivided into smaller ones, such as the following.

i. What characteristics of DNA replication are compromised by centrosome disruption?

ii. What is the underlying mechanism behind the observed effect?

iii. How is MK2 activated upon centrosome disruption?

iv. Which downstream component(s) of the P38-MK2 pathway is activated by centrosome disruption?

v. Which component(s) of the pathway, if any, are located at the centrosome?

vi. Finally, how do PLK4 overexpression and supernumerary centrosomes affect DNA replication? 


\section{MATERIALS AND METHODS}

\section{i. Technical devices}

\begin{tabular}{|c|c|}
\hline Device & Manufacturer \\
\hline Blotting chamber & Biozym \\
\hline Centrifuge 5415R & Eppendorf \\
\hline Centrifuge $5810 \mathrm{R}$ & Eppendorf \\
\hline Chemiluminescence imager Chemocam HR 163200 & Intas Science Imaging Instruments \\
\hline Chemiluminescence imager Chemidoc XRS+ & Biorad \\
\hline Electrophoresis system for SDS-PAGE & Amersham Biosciences \\
\hline Laminar flow cabinet Hera safe & Heraeus, Thermo Scientific \\
\hline Light microscope Axovert 40C & Zeiss \\
\hline Microscope, Axio Scope.A1 & Zeiss \\
\hline PCR machine Thermocycler T Personal & Biometra \\
\hline pH meter inoLab & WTW GmbH \\
\hline Pipets, Eppendorf Research & Eppendorf \\
\hline Power Supply & Biometra \\
\hline Roller RM5-30V & CAT \\
\hline Scales Acculab ALC-6100.1 & Sartorius \\
\hline Scales LE623S & Sartorius \\
\hline Scanner CanoScan 8600F & Canon \\
\hline Sonication device Bioruptor & Diagenode \\
\hline Spectrophotometer NanoDrop ND-1000 & PeqLab \\
\hline Thermomixer Comfort & Eppendorf \\
\hline Vortex-Genie 2 & Scientific Instruments \\
\hline FACS & BD FACSCanto II \\
\hline FACS & GuavaEasy Cyte Plus \\
\hline
\end{tabular}




\section{ii. Consumables}

\section{Consumable}

96-well plate for qPCR

Cell culture dishes $(10 \mathrm{~cm}, 15 \mathrm{~cm})$

Bacteria culture dish $(10 \mathrm{~cm})$

Cell culture plate (6-well, 12-well)

Cell culture plate 24-well

Cell scraper $(16 \mathrm{~cm}, 25 \mathrm{~cm})$

Coverslips

Cryo-tubes for cell freezing

Glass pipets $(5 \mathrm{ml}, 10 \mathrm{ml}, 25 \mathrm{ml})$

Glass Slides Superfrost

Parafilm

Pipet tips (10 $\mu \mathrm{L}, 20-200 \mu \mathrm{L}, 1,000 \mu \mathrm{L})$

Protran nitrocellulose transfer membrane

Reaction tube $(0.5 \mathrm{~mL}, 1.5 \mathrm{~mL}, 2.0 \mathrm{~mL})$

Reaction tube (15 mL, $50 \mathrm{~mL})$

Safe-lock reaction tube $(1.5 \mathrm{~mL})$

Sterile filter $(0.2 \mu \mathrm{M}$ and $0.45 \mu \mathrm{M})$

Syringe canula

Whatman paper

\section{Manufacturer}

4titude

Greiner

Sarstedt

Greiner

Costar

Sarstedt

Menzel, Roth

Nunc

Sarstedt

Menzel

Sigma-Aldrich

Greiner

Whatman

Eppendorf

Greiner

Eppendorf

Millipore

B.Braun

Whatman 


\section{iii. Chemicals and reagents}

\begin{tabular}{|c|c|}
\hline Chemical & Manufacturer \\
\hline Acetic acid & Roth \\
\hline Agar & Sigma-Aldrich \\
\hline Agarose & Roth \\
\hline Albumin Fraction V (Bovine Serum Albumin, BSA) & Roth \\
\hline Ammonium persulfate (APS) & Roth \\
\hline Ampicillin & Roth \\
\hline Chlorodeoxyuridine (CldU) & Sigma-Aldrich \\
\hline Chloroform & Roth \\
\hline Deoxynucleotide triphosphates (dNTPs) & Primetech \\
\hline Dimethyl sulfoxide (DMSO) & AppliChem \\
\hline DNA ladder GeneRuler & Fermentas \\
\hline Ethanol $99.8 \%$ & Roth \\
\hline Formaldehyde, $37 \%$ solution & Roth \\
\hline Glycerol >99\% p.a. & Roth \\
\hline Glycine $>99 \%$ p.a. & Roth \\
\hline Hydrogen chloride $(\mathrm{HCl})$ & Roth \\
\hline lododeoxyuridine (IdU) & Sigma-Aldrich \\
\hline Isopropanol & Roth \\
\hline Lipofectamine 2000/3000 & Invitrogen \\
\hline Methanol >99\% (MetOH) & Roth \\
\hline Milk powder & Roth \\
\hline Nailpolish & essence \\
\hline Nuclease-free $\mathrm{H}_{2} \mathrm{O}$ & Ambion \\
\hline
\end{tabular}


PageRuler Prestained Protein Ladder

Pefablock SC protease inhibitor

Pepstatin A

Ponceau S

Potassium Chloride

Potassium Hydrogenphosphate $\left(\mathrm{KH}_{2} \mathrm{PO}_{4}\right)$

Random hexamer primers

Rotiphorese Gel 30

Sodium (di-)hydrogenphosphate dihydrate $\left(\mathrm{Na}_{2} \mathrm{HPO}_{4}\right) \times 2 \mathrm{H}_{2} \mathrm{O}$

Sodium chloride $(\mathrm{NaCl})$

Sodium deoxycholate

Sodium dodecyl sulfate (SDS)

Sodium dodecyl sulfate (SDS)

Sodium ethylene diamine tetra-acetic acid (Na-EDTA)

Sodium hydrogen phosphate monohydrate $\left(\mathrm{NaHPO}_{4} \times \mathrm{H}_{2} \mathrm{O}\right)$

Sodium hydroxide $(\mathrm{NaOH})$

SYBR green

Tetramethylethylenediamine (TEMED)

Trehalose dehydrate

Trisamine (Tris) Pufferan >99\% p.a.

Triton-X100

TRIZOL

Tween 20

Vectashield mounting medium
Fermentas

Roth

AppliChem

Roth

AppliChem

Roth

Thermo Scientific

Roth

Roth

Roth

AppliChem

Roth

BioRad

Roth

Roth

Sigma-Aldrich

Invitrogen

Merck

USB Corp.

Roth

AppliChem

Invitrogen

Applichem

Vector Laboratories 


\section{iv. Buffers and Solutions}

\begin{tabular}{ll}
\hline \multicolumn{2}{l}{ Cell lysis buffer } \\
\hline \\
urea \\
RIPA lysis buffer & $2.5 \mathrm{M}$ \\
Pefa & $60 \%$ \\
PA & $1 \%$ \\
L/A & $1 \%$ \\
\hline
\end{tabular}

\begin{tabular}{ll}
\hline Ponceau S & \\
\hline & \\
Ponceau S & $0.5 \%$ \\
acetic acid & $1 \%$ \\
in $\mathrm{ddH}_{2} \mathrm{O}$ & \\
\hline
\end{tabular}

\begin{tabular}{ll}
\hline \multicolumn{2}{l}{ Fibre Assay Blocking Solution } \\
\hline & \\
BSA & $3 \%$ \\
Tween20 & $0.1 \%$ \\
in PBS pH 7.4 & \\
\hline
\end{tabular}

\begin{tabular}{ll}
\hline RIPA Lysis Buffer & \\
\hline & \\
Triton X-100 & $1 \%$ \\
Na-deoxycholate & $1 \%$ \\
$\mathrm{NaCl}$ & $150 \mathrm{mM}$ \\
EDTA & $10 \mathrm{mM}$ \\
Tris, $\mathrm{pH} 7.5$ & $20 \mathrm{mM}$ \\
in $\mathrm{ddH}_{2} \mathrm{O}$ & \\
\hline
\end{tabular}

\begin{tabular}{ll}
\hline \multicolumn{2}{l}{ Fibre Assay Fixative } \\
\hline \\
MeOH & $75 \%$ \\
Acetic Acid & $25 \%$ \\
\hline
\end{tabular}

\begin{tabular}{ll}
\hline \multicolumn{2}{l}{ Fibre Assay Spreading Buffer } \\
\hline & \\
Tris pH 7.4 & $200 \mathrm{mM}$ \\
EDTA & $50 \mathrm{mM}$ \\
SDS in $\mathrm{ddH}_{2} \mathrm{O}$ & $0.5 \%$ \\
\hline
\end{tabular}

\begin{tabular}{ll}
\hline 6x Laemmli Buffer & \\
\hline & \\
Tris pH 6.8 & $0.35 \mathrm{M}$ \\
glycerine & $30 \%$ \\
SDS & $10 \%$ \\
dithiothreitol & $9.3 \%$ \\
bromophenol blue & $0.02 \%$ \\
in $\mathrm{ddH}_{2} \mathrm{O}$ & \\
\hline
\end{tabular}

\begin{tabular}{ll}
\hline \multicolumn{2}{l}{ Phosphate Buffered Saline (PBS) } \\
\hline & \\
$\mathrm{NaCl}$ & $24 \mathrm{mM}$ \\
$\mathrm{KCl}$ & $0.27 \mathrm{mM}$ \\
$\mathrm{Na}_{2} \mathrm{HPO}_{4} \times 7 \mathrm{H}_{2} \mathrm{O}$ & $0.81 \mathrm{mM}$ \\
$\mathrm{KH}_{2} \mathrm{PO}_{4}$ & $0.15 \mathrm{mM}$ \\
in $\mathrm{ddH}_{2} \mathrm{O}$ & \\
\hline
\end{tabular}

\begin{tabular}{ll}
\hline $\begin{array}{l}\text { Tris Buffered Saline (TBS) + Tween20 } \\
\text { (TBS-T) }\end{array}$ & \\
\hline & $50 \mathrm{mM}$ \\
Tris & $150 \mathrm{mM}$ \\
$\mathrm{NaCl}$ & $0.1 \%$ \\
Tween20 & \\
in $\mathrm{ddH}_{2} \mathrm{O}$ & \\
\hline
\end{tabular}

\begin{tabular}{ll}
\hline Western Blot Transfer Buffer \\
\hline & \\
Tris & $25 \mathrm{mM}$ \\
glycin & $192 \mathrm{mM}$ \\
$\mathrm{MeOH}$ & $20 \%$ \\
in ddH & $\mathrm{O}$ \\
\hline
\end{tabular}

\begin{tabular}{ll}
\hline Buffer A (Dot blot) & \\
\hline & \\
$\mathrm{NaCl}$ & $0.1 \mathrm{M}$ \\
EDTA & $1 \mathrm{mM}$ \\
EGTA & $0.5 \mathrm{mM}$ \\
HEPES & $50 \mathrm{mM}$ \\
\hline
\end{tabular}




\begin{tabular}{ll}
\hline Buffer B (Dot blot) & \\
\hline & \\
Triton X-100 & $0.25 \%$ \\
EDTA & $10 \mathrm{mM}$ \\
EGTA & $0.5 \mathrm{mM}$ \\
HEPES & $20 \mathrm{mM}$ \\
\hline
\end{tabular}

\section{Buffer C (Dot blot)}

\begin{tabular}{ll}
$\mathrm{NaCl}$ & $0.15 \mathrm{M}$ \\
EDTA & $1 \mathrm{mM}$ \\
EGTA & $0.5 \mathrm{mM}$ \\
HEPES & $50 \mathrm{mM}$ \\
\hline
\end{tabular}

\begin{tabular}{ll}
\hline $\begin{array}{l}\text { Buffer } 5 X \\
\text { (Dot blot) }\end{array}$ \\
\hline \\
Triton X-100 & $5 \%$ \\
NaCl & $0.75 \mathrm{M}$ \\
EDTA & $5 \mathrm{mM}$ \\
EGTA & $2.5 \mathrm{mM}$ \\
\hline
\end{tabular}

\section{v. Enzymes and reaction buffers}

\begin{tabular}{lll}
\hline Enzyme & Buffer & Producer \\
\hline M-MuLV reverse transcriptase & $10 x$ MuLV buffer & New England Biolabs (NEB) \\
& $\left.\begin{array}{l}10 \times \text { Taq buffer }(+\mathrm{KCl},- \\
\text { Taq DNA Polymerase for qPCR }\end{array} \mathrm{MgCl}_{2}\right)$ & Primetech, Fermentas \\
\hline
\end{tabular}

\section{vi. Commercial Kits}

\begin{tabular}{ll}
\hline Name & Producer \\
\hline Immobilon Western HRP Substrate Peroxide Solution & Millipore, Merck \\
Pierce BCA Protein assay kit & Thermo Fisher \\
PureYield Plasmid Midiprep System & Promega \\
SuperSignal Western Femto Maximum Sensitivity Substrate & Thermo Fisher \\
Click-iT ${ }^{\mathrm{TM}}$ EdU Alexa Fluor ${ }^{\mathrm{TM}} 647$ Imaging Kit & ThermoFisher \\
\hline
\end{tabular}




\section{vii. Chemotherapeutics and Pharmacological inhibitors}

\begin{tabular}{ll}
\hline Name & Producer \\
\hline Centrinone B, Plk4 inhibitor & MedChem Express \\
Gemcitabine $\left(2^{\prime}, 2^{\prime}\right.$-difluorodeoxycytidine $\left.(\mathrm{dFdC})\right)$ & Calbiochem \\
MK2III (MK2 inhibitor) & Calbiochem \\
U0126 Inhibitor & Cell signaling \\
CFI-400945 & Cayman chemical \\
Thymidine & \\
PD 0332991 isethionate (CDK4i) & Sigma \\
LDC00003 -CDK9 I & Sigma \\
Dimethylenastron DME & Selleckchem \\
MLK3 inhibitor (URMC-099) & Sigma \\
\hline
\end{tabular}

\section{viii. Oligonucleotides}

\begin{tabular}{llc}
\hline siRNA & Manufacturer & Identifier \\
\hline Silencer select negative control \#1 & Ambion & 4390844 \\
Silencer select negative control \#2 & & 4390846 \\
\hline & Ambion & s22796 \\
Silencer Select siRNA Cep152-1 & & $\mathrm{s} 225922$ \\
Silencer Select siRNA Cep152-2 & & $\mathrm{s} 225923$ \\
Silencer Select siRNA Cep152-3 & & \\
\hline Silencer Select siRNA CCP110-1 & Ambion & $\mathrm{S18787}$ \\
Silencer Select siRNA CCP110-2 & & $\mathrm{S} 18786$ \\
Silencer Select siRNA CCP110-3 & & $\mathrm{S} 18785$ \\
\hline
\end{tabular}




\section{MATERIALS AND METHODS}

Silencer Select siRNA SASS6-1

Ambion

S46486

Silencer Select siRNA SASS6-2

S46485

Silencer Select siRNA SASS6-3

S46487

Silencer Select siRNA CEP192-1

S226819

Silencer Select siRNA CEP192-2

Ambion

S226820

Silencer Select siRNA CEP192-3

S302229

Silencer Select siRNA MK2_1

Ambion

s569

Silencer Select siRNA MK2_2

s570

Silencer Select siRNA MK2_3

s571

Silencer Select siRNA CJUN-1

Silencer Select siRNA CJUN-2

Ambion

s7658

Silencer Select siRNA CJUN-3

s7659

s7660

s8816

Silencer Select siRNA MAP3K11-1

Silencer Select siRNA MAP3K11 -2

Ambion

s8815

Silencer Select siRNA MAP3K11 -3 s8814 


\section{ix. Plasmids}

\begin{tabular}{llc}
\hline Name & Source & Identifier \\
\hline pcDNA3 & Invitrogen & \\
pFRT- & & \\
TODestFLAGHA_RNAseH1 \\
pFRT- \\
TODestFLAGHA_RNAseH1 was \\
$\begin{array}{l}\text { a gift from Thomas Tuschl } \\
\begin{array}{l}\text { PFRT-ToDestFlag-HA } \\
\text { pFRT_TO_DESTFLAGHA was a } \\
\text { gift from Thomas Tuschl }\end{array}\end{array}$ \\
$\begin{array}{l}\text { pcDNA3 PIk4(Sak) wt (Nigg } \\
\text { HR9) } \\
\text { pcDNA3 Plk4(Sak) wt (Nigg HR9) } \\
\text { was a gift from Erich Nigg }\end{array}$ & Addgene & \\
\hline
\end{tabular}

\section{x. Antibodies}

\begin{tabular}{lcc}
\hline Antibody & Source & Identifier \\
$\begin{array}{l}\text { Anti-Pericentrin antibody [mAbcam } \\
28144] \text { - Centrosome Marker }\end{array}$ & Abcam & ab28144 \\
Cep152 & Sigma & HPA039408 \\
$\begin{array}{l}\text { phospho-H2A.X (Ser139) clone 20E3 } \\
\text { rabbit }\end{array}$ & Cell Signaling & 9718 \\
$\begin{array}{l}\text { Phospho-HSP27 (Ser82) Antibody } \\
\text { p38 MAPK Antibody }\end{array}$ & Cell Signalling & $2401 \mathrm{~L}$ \\
$\begin{array}{l}\text { ATF2 Antibody (F2BR-1) } \\
\text { MAPKAPK-2 Antibody }\end{array}$ & Cell Signalling & $9212 \mathrm{~S}$ \\
& Santa Cruz & sc-242 \\
$\begin{array}{l}\text { Phospho-MAPKAPK-2 (Thr334) } \\
\text { (27B7) }\end{array}$ & Cell signaling & $3042 S$
\end{tabular}




\begin{tabular}{|c|c|c|}
\hline $\begin{array}{l}\text { rat anti-BrdU [BU1/75 (ICR1)] - no } \\
\text { longer available }\end{array}$ & AbD Serotec & MCA2060 (AB_323427) \\
\hline rat anti-BrdU [BU1/75 (ICR1)] & Abcam & ab6326 (AB_305426) \\
\hline mouse anti-BrdU clone B44 & BD-Bioscience & 347580 (AB_10015219) \\
\hline $\begin{array}{l}\text { Alexa Fluor 488-conjugated goat anti- } \\
\text { rat antibody }\end{array}$ & ThermoFisher & A11029 (AB_138404) \\
\hline $\begin{array}{l}\text { Alexa Fluor 555-conjugated goat anti- } \\
\text { rat antibody }\end{array}$ & ThermoFisher & A21434 (AB_141733) \\
\hline S9.6 antibody & Absolute antibody & Ab01137-2.0 \\
\hline Anti-MLK3 antibody [EP1460Y] & Abcam & ab51068 \\
\hline RNAseH1 Antibody & Abcam & ab56560 \\
\hline PLK4 antibody & Proteintech & 12952-1-AP \\
\hline RNA Polymerase II (N-20) & SantaCruz & sc-899 \\
\hline Phospho-ATF-2 (Thr71) Antibody & Cell Signaling & $9221 S$ \\
\hline C-Jun & Abcam & ab32137 \\
\hline P-C-Jun (S63) & Cell Signaling & 9261 \\
\hline Hsc70 (B-6) & Santa Cruz & sc-7298 \\
\hline Histone H3 & Abcam & ab1791 \\
\hline Histone H3 (acetyl K27) & Abcam & ab4729 \\
\hline MCM7 (D10A11) XP & Cell Signaling & 3735 \\
\hline GAPDH (6C5) & Abcam & ab8245 \\
\hline ssDNA MS X HU & Merck & 2684913 \\
\hline
\end{tabular}




\section{xi. Secondary Antibodies for Western Blot}

\begin{tabular}{llc}
\hline Antibody & Manufacturer & Catalog Number \\
\hline $\begin{array}{l}\text { HRP-coupled AffiniPure } F\left(a b^{\prime}\right) 2 \\
\text { fragment, anti-mouse IgG }(\mathrm{H}+\mathrm{L})\end{array}$ & Jackson Immunoresearch & $711-036-152$ \\
$\begin{array}{l}\text { HRP-coupled AffiniPure } F\left(\mathrm{ab}^{\prime}\right) 2 \\
\text { fragment, anti-rabbit IgG }(\mathrm{H}+\mathrm{L})\end{array}$ & Jackson Immunoresearch & $715-036-150$ \\
Alexa-Fluor-488 goat anti-mouse & Invitrogen, Life Technologies & A-11017 \\
& & \\
Alexa-Fluor-555 goat anti-mouse & Invitrogen, Life Technologies & A-11003 \\
Alexa-Fluor-647 goat anti-mouse & Învitrogen, Life Technologies & A-21236 \\
\hline
\end{tabular}

\section{xii. Cell culture}

\section{Cell line}

H1299

SW48-WT

SW48-KRAS-G12D

SW48-KRAS-G12V

SW48-KRAS-G13D

RPE-KRAS-P53KO-WT

RPA-KRAS-P53KO-G12D

Skin fibroblast -1

Skin fibroblast-2

Seckele syndrome cells

HCT116 p53-/-

HCT116 p53+/+ 


\section{xiii. Media and reagents for cell culture}

\begin{tabular}{ll}
\hline Reagent & Manufacturer \\
\hline Ciprofloxacin & Bayer \\
DMEM powder & Gibco, Life Technologies \\
FCS & Gibco, Life Technologies \\
L-glutamine & Gibco, Life Technologies \\
PBS (tablets) & Gibco, Life Technologies \\
Penicillin/Streptomycin & Gibco, Life Technologies \\
Puromycin & Gibco, Life Technologies \\
RPMI 1640 w Hepes w/o Glut & Gibco, Life Technologies \\
Tetracyclin & Gibco, Life Technologies \\
Trypsin/EDTA & Gibco, Life Technologies \\
\hline
\end{tabular}

Dulbecco's Modified Eagle's Medium (DMEM)

\begin{tabular}{ll}
\hline $\mathrm{DMEM}$ powder & $10 \mathrm{~g} / \mathrm{l}$ \\
$\mathrm{NaHCO}_{3}$ & $3.7 \mathrm{~g} / \mathrm{l}$ \\
$\mathrm{HEPES}$ & $5.96 \mathrm{~g} / \mathrm{l}$ \\
\hline
\end{tabular}

\section{Dulbecco's Modified Eagle's Medium (DMEM) with supplements}

\section{DMEM}

FCS

$10 \%$

Penicillin/Streptomycin (Pen/Strep)

$50 \mathrm{U} / \mathrm{ml}$

L-glutamine

$200 \mu \mathrm{M}$

Ciprofloxacin

$10 \mu \mathrm{g} / \mathrm{ml}$

in $\mathrm{ddH}_{2} \mathrm{O}$ 
RPMI with supplements

RPMI

FCS

$10 \%$

Penicillin/Streptomycin (Pen/Strep)

$50 \mathrm{U} / \mathrm{ml}$

L-glutamine in $\mathrm{ddH}_{2} \mathrm{O}$

$200 \mu \mathrm{M}$

\section{xiv. Bacteria}

Bacterial strains used for plasmid amplification

Bacteria strain

DH10B ${ }^{\mathrm{TM}}$ chemically competent cells

\section{Source}

Thermo Scientific

\section{Bacteria growth medium}

\begin{tabular}{ll}
\hline 2YT medium & \\
\hline Tryptone & $1.6 \%$ \\
yeast extract & $1 \%$ \\
$\mathrm{NaCl}$ & $0.5 \%$ \\
\hline
\end{tabular}

\section{YT agar}

YT agar

2YT medium

$15 \%$

$100 \%$

\section{xv. Software and Databases}

\begin{tabular}{ll}
\hline Name & Manufacturer \\
\hline Axio Vision & Zeiss \\
Excel & Microsoft \\
GraphPad Prism & GraphPad Software \\
Image Lab 5.2.1 & Biorad \\
ImageJ & General Public License \\
NanoDrop Software & Peqlab \\
\hline
\end{tabular}




\section{METHODS}

\section{i. Cell culture}

Cells were cultivated at $37^{\circ} \mathrm{C}, 5 \% \mathrm{CO}_{2}$. $\mathrm{H} 1299$, RPE-P53KO (WT-G12D) cells were cultured in DMEM (Dulbecco's Modified Eagle Medium) supplied with 10\% FBS (fetal bovine serum), $2 \mathrm{mM}$ penicillin, $2 \mathrm{mM}$ streptomycin and $10 \mu \mathrm{g} / \mathrm{mL}$ ciprofloxacin. SW48 (WT-G12D, G13D, G12V) was cultured in Mayco's medium supplied with 10\% FBS (fetal bovine serum), $2 \mathrm{mM}$ penicillin, and $2 \mathrm{mM}$ streptomycin. Seckel and fibroblast cells were cultivated with DMEM Glutamax 20\% FBS (fetal bovine serum), $2 \mathrm{mM}$ penicillin, $2 \mathrm{mM}$ streptomycin, and $10 \mu \mathrm{g} / \mathrm{mL}$ ciprofloxacin. For cell, harvesting, cells were washed with pre-warmed PBS, followed by the addition of $0.1 \%$ trypsin/EDTA for 5 minutes at $37^{\circ} \mathrm{C}$. Once cells detached, the reaction was stopped by the addition of the FCS-containing culture medium. Cells were either counted and reseeded again for experimental purposes or re-seeded at dilutions between 1:10 two times per week. Seckel cells and the fibroblast cell medium were changed every second day, and splitting was once a week. All cell culture work was carried out under sterile conditions.

\section{ii. Cell transfections}

Knocking down gene expression in human cells, a reverse transfection of cationic liposome formulation Lipofectamine 3000 was performed. A transfection mix with a final 10nM concentration siRNA was prepared by separately incubating siRNA (Solution A) and Lipofectamine (Solution B) with DMEM without supplements. The two separate solutions were incubated at room temperature for 5 minutes. Solution B was added to solution A, followed by an additional 20-minute incubation. Cells were seeded with the transfection mix into medium containing supplements. Culture medium was exchanged after 24 hours, and experiments were carried out 48-72 hours post siRNA transfection. Overexpressing DNA plasmid, a forward transfection protocol was used according to the manufacturer's instructions. For this, a transfection mix was prepared from $2 \mu \mathrm{g}$ of plasmid DNA (solution A) and a second mix containing Lipofectamine 2000 (solution B) in DMEM without supplements incubated for 5 minutes separately, then they were combined (solutions B \& A) and incubated for 15 minutes. The 
transfection mix was added drop-wise onto adherent cells. Media was changed after 6-7 hours, and experiments carried out 30 hours post-transfection.

\section{iii. Chemical Treatments}

Several pharmacological inhibitors used for cell treatments were dissolved either with DMSO or water and aliquots prepared according to the manufacturer's instructions. Treatments were prepared by adding the drug to a pre-warmed medium. For control samples, the respective solvent was added instead of the drug.

\section{iv. Cell synchronization}

The synchronization of $\mathrm{H} 1299$ cells at the G1/S transition was carried using the CDK4 inhibitor and Thymidine. H1299 cells were treated with CDK4 inhibitor Palbociclib for 24 hours, followed with $500 \mathrm{nM}$ Centrinone B for another 24 hours. CDK4 inhibitor was washed 16 hours prior harvesting and replaced by a thymidine block with Centrinone B. 48 hours after treating the cells with $500 \mathrm{nM}$ Centrinone B. Cells were released by removing from Thymidine for 3-4 hours before harvesting.

\section{v. Flow cytometry}

Cell cycle analysis was carried as described in Figure 6.3. Cells were treated as indicated in the previous section of cell synchronization and as in Figure 6.3. Post-treatment cells were detached and fixed with cold ethanol at $-20^{\circ} \mathrm{C}$ for overnight. Samples were centrifuged, and the pellet rehydrated with PBS for 10 minutes. After centrifugation, the cell pellet was resuspended in PBS with RNase A $(200 \mu \mathrm{g} / \mathrm{ml})$ and incubated at $37^{\circ} \mathrm{C}$ for 30 minutes. Propidium iodide staining was performed with a $30 \mu \mathrm{g} / \mathrm{ml}$ solution for 5-15 minutes at room temperature in the dark. Cells were analyzed using a Guava $\AA^{\circledR}$ Express Pro EasyCyte flow cytometer and Cyto Soft 5.3 software (Merck). Measurements were taken by counting 10000 events at 300-700 cells $/ \mathrm{mL}$. To determine the number of gated cells in each cell cycle phase (G1, S, and G2/M), histogram markers were adjusted to the asynchronous cell FACS profile. The average values of duplicates were determined for each cell cycle phase. 


\section{vi. Cell proliferation assay (Celigo)}

For confluence measurement, cells were seeded at a density of 5000-10000 cells/well in 24-well plates. Cells were treated as indicated in Figures 6.4, 6.6 and 6.9. Their proliferation capacity was measured using the CeligoTM Cytometer (Nexcelom, software version 2.0). Cell confluence was measured every 24 hours for up to 7 days. Experiments were carried out in 2 biological replicates, and with six technical; the average of those was plotted on a graph using GraphPad Prism.

\begin{tabular}{|c|c|}
\hline Parameter & Meaning \\
\hline Intensity threshold & 15 \\
\hline Precision & High \\
\hline Diameter & 30 \\
\hline Minimum thickness & 3 \\
\hline
\end{tabular}

\section{vii. Proteins separation by SDS-PAGE}

Proteins were separated according to their molecular sizes using SDS-PAGE (Sodium dodecyl sulfate-polyacrylamide gel electrophoresis. Equal amounts of protein samples were loaded and separated by 10\% SDS-PAGE. Electrophoresis was carried out with a constant voltage of $80 \mathrm{~V}$ for stacking and $100 \mathrm{~V}$ for separation. Finally, samples were transferred onto the nitrocellulose membrane on ice for 2 hours at $100 \mathrm{~V}$.

\section{viii. Immunoblotting}

Cells were harvested in protein lysis buffer with proteinase inhibitors (pepstatin, leupeptin hemisulfate, aprotinin). The samples were sonicated to disrupt DNA-protein complexes. Total protein concentration was measured using a Pierce BCA Protein assay kit (Thermo Scientific Fisher). After boiling the samples in Laemmli buffer at $95^{\circ} \mathrm{C}$ for 5 minutes, equal amounts of protein samples were separated by SDS-PAGE, transferred onto nitrocellulose, and incubated with primary antibodies of the following proteins: MLK3 (D-11) (Santa Cruz), PLK4 (Proteintech), p-p38 (E-1) (Santa Cruz), p-MK2 (Cell signaling), pHsp27 S82 (Cell signaling), GAPDH (Abcam), RNAseH1 (Abcam), HSC70 (Santa Cruz), H3K27me3 (Abcam), H3K27ac (Abcam), Histone H3 (Abcam), P-JUN (S63) (Cell signaling), JUN (Abcam), TBP (Santa Cruz), ATF2 (Santa Cruz), 
Phospho-ATF2 (T71) (Cell signaling), RNA Polymerase II (N-20) (Santa Cruz), MCM7 (D10A11) XP (Cell signaling), MAPKAPK-2 (Cell signaling), P53 (Cell signaling), phospho-H2A.X (Ser139) (Cell signaling). To visualize specific proteins on the membrane were incubated with secondary conjugation antibody to horseradish peroxidase (HRP). Protein level was visualized by the Intas ChemoStar Imager Software and the Image Lab 5.2.1 Software by Biorad.

\section{ix. RNA extraction and real-time quantitative PCR}

Total RNA was extracted from cells using TRIzol® (Invitrogen). mRNA was prepared by reverse-transcribed using Moloney Murine Leukemia Virus reverse transcriptase and random hexamer primers (Thermo Scientific). qRT-PCR analysis using SYBR Green (Invitrogen) was followed. Gene expression levels were normalized to the mRNA encoding HPRT1 or 36B4, and the analysis was conducted using the $\Delta \Delta \mathrm{Ct}$ method.

\section{R-loop detection}

Cells were grown on coverslips overnight prior to transfection / treatment, washed once with phosphate-buffered saline (PBS), and fixed either by cold methanol at -20C for 20 minute or with $4 \%$ paraformaldehyde for 10 minute at room temperature followed by permeabilization with 0.5\% Triton X-100 in PBS for 30 minute, three brief washes with PBS containing $0.1 \%$ Tween 20 (PBST) and blocking with 3\% bovine serum albumin (BSA) in PBST for 1 hour. The primary antibody S9.6 (Absolute antibody) was diluted in the blocking buffer (1:100) and incubated overnight at $4^{\circ} \mathrm{C}$, followed by incubation with 488 Alexa Fluor-coupled donkey anti-mouse IgG $(\mathrm{H}+\mathrm{L})$ (Invitrogen) 1:250 for 2 hours at room temperature. Coverslips were washed with PBST and were briefly incubated with 1:2000 DAPI (4', 6-diamidino-2-phenylindole), followed by mounting (DAKO). Fluorescence signals were detected by a microscope (Zeiss Axio Scope.A1) equipped with filters for 488nm, an EC Plan-Neofluar 100x oil objective, and an Axiocam 503 color camera. Per condition, approximately 15- 20 images were taken with the AxioVision software and analyzed using ImageJ.

\section{xi. Immunofluorescence analysis of centrosomes}

Two thousand cells were seeded on eight well chamber slides (Nunc/Thermo, cat \#177445) overnight before treatment. Cells were treated with 300nM Centrinone B or DMSO for 48 hours. The slide was washed carefully once with phosphate-buffered saline (PBS), fixed and 


\section{MATERIALS AND METHODS}

stained as above. Antibodies were used as follows: 1:200 PCNT (Abcam), 1:50 CEP152 (Sigma), 1:100 MLK3 (Abcam), 1:500 of 488 Alexa fluor Donkey anti-mouse, 1:500 594 Alexa fluor goat anti-rabbit, 1:2000 Hoechst 33342.

\section{xii. Chromatin fractionation}

Cells were synchronized using the CDK4 inhibitor Palbociclib and released into a thymidine block as described in the synchronization sections (Figure 2A). Forty-eight hours after treating the cells with $500 \mathrm{nM}$ Centrinone $B$, the cells were trypsinized and collected in $15 \mathrm{ml}$ tubes, washed twice with PBS, and centrifuged at $100 \mathrm{~g}$ for 5 minutes. The pellets were resuspended in $1 \mathrm{ml}$ Buffer A (10mM HEPES, PH 7.9, 10mM KCL, 1.5mM MgCl2 0.34M Sucrose 10\% Glycerol, $1 \mathrm{mM}$ dithiothreitol, and protease inhibitors cocktails (COmplete Roche) and transferred into 1.5 $\mathrm{ml}$ tubes. Triton X-100 was added to each tube to a final concentration of $0.1 \%$ and incubated on a rotating wheel for 15 minutes at $4^{\circ} \mathrm{C}$. The tubes were centrifuged at $1300 \mathrm{~g}$ for 5 minutes at $4^{\circ} \mathrm{C}$. The supernatant (cytoplasmic fraction) was transferred to new tubes. The pellets were washed once with Buffer $A$ and then further lysed with $250 \mu$ modified RIPA buffer (1mM EDTA, 150mM $\mathrm{NaCl}, 0.1 \% \mathrm{Na}-\mathrm{DOC}, 1 \% \mathrm{NP}-40,50 \mathrm{Mm}$ Tris $\mathrm{pH} 7.5$, and protease inhibitors cocktails- COmplete Roche). $50 \mathrm{U}$ of benzonase (nuclease; Novagen) was added to the samples and incubated for 510 minutes at room temperature. Samples were mixed by pipetting during the incubation time until they lost viscosity. Samples were diluted 1:3 with a modified RIPA buffer. The supernatant was cleared by centrifuging the samples for 7 minutes at $16000 \mathrm{~g}, 4^{\circ} \mathrm{C}$, and the clear chromatin fraction was transferred to a new tube. After boiling the samples in Laemmli buffer at $95^{\circ} \mathrm{C}$ for 5 minutes, equal amounts of protein samples were separated by SDS-PAGE, transferred onto nitrocellulose, and visualized with the following antibodies: JUN (Santa Cruz), ATF2 (Cell signaling), MCM7 (Cell signaling), GAPDH (Abcam).

\section{xiii. EdU click reaction}

Both synchronized and non-synchronized H1299 cells were treated with $500 \mathrm{Nm}$ were incubated with $10 \mu \mathrm{M}$ EdU two hours before harvesting cells. Two hours post labeling the cells medium was removed, and cells were washed with 1X PBS. Cells were fixed with $3.7 \%$ PFA in PBS for 30 minutes on RT. Cells were permeabilizated with $0.5 \%$ Triton $X-100$ for 30 minutes on RT, followed by two times washes with PBS. Staining and detecting the EdU incorporation into DNA was detected using the Click-iT ${ }^{\mathrm{TM}}$ EdU Alexa Fluor ${ }^{\circledR}$ imaging kit (Invitrogen/Molecular Probes, Eugene, OR). 
xiv. Dot blot analysis of RNA: DNA hybrids

H1299 cells were synchronized, as in Figure 2A. Forty-eight hours after treating the cells with $500 \mathrm{nM}$ Centrinone B or 2 hours with $5 \mu \mathrm{M}$ etoposide, the cells were fixed with $1.1 \%$ PFA in Buffer $A$ for 30 minutes, followed by quenching with glycine. The cells were then harvested in Buffer B, then resuspended in Buffer C. 150 $\mu \mathrm{L} 1 \mathrm{X}$ IB Buffer was added to the pellet. Samples were sonicated (Bioruptor, ten cycles, $30 \mathrm{sec}$ on, and $30 \mathrm{sec}$ off) and centrifugation, the supernatant was treated with proteinase $\mathrm{K}$ at $50^{\circ} \mathrm{C}$ for one hour, phenol-extracted and ethanolprecipitated. 500ng of the DNA in $2 \mu \mathrm{L}$ was spotted onto the pre-wet nitrocellulose membrane and cross-linked with UV-C. The membrane was blocked with 5\% BSA in PBS-T $(0.25 \%$ Tween-20) and incubated with S9.6 antibody (1:300 in 5\% BSA in PBS-T) for 16 hours at $4^{\circ} \mathrm{C}$, followed by secondary antibodies. For normalization and in parallel, the DNA was denatured with $2.5 \mathrm{M} \mathrm{HCl}$ for 10 minutes, washed with PBS-T, and incubated with antibody to ssDNA (1:1000) followed by secondary antibody. The peroxidase-coupled secondary antibodies were detected by luminescence.

\section{xv. Chromosome spread analysis and chromosome counting}

H1299 cells were seeded in 6-well plates, as in Figure 6.3. The following day, cells were treated with DMSO or Centrinone B and further incubated for two days or seven days. On the day of harvesting, cells were treated with $2 \mu \mathrm{M}$ Dimethylenastron DME (Sigma) for 4.5 hours. The cells were then trypsinized and resolved in $750 \mu \mathrm{L}$ hypotonic solution (40\% medium in water) for 10-15 minutes. $250 \mu \mathrm{L}$ of Carnoy's fixative solution (MeOH-glacial acetic acid (3:1)) was added to the pellet. The cells were then resuspended twice in Carnoy's fixative and stored at $-20^{\circ} \mathrm{C}$ for 16 hours. After resuspension in acetic acid, $10 \mu$ of cell suspension was dropped on cold glass slides from $\sim 2 \mathrm{~m}$ height. Slides were placed on a $42{ }^{\circ} \mathrm{C}$ heat block for $\sim 10-15$ minutes and then stained with $8 \%$ Giemsa solution for 15 minutes. The analysis of chromosomes was carried out by transmission microscopy.

\section{xvi. DNA Fiber Assay}

DNA fiber assay was our way to study replication speed and progression in cells subjected to centrosomes impairment. The foremost step in this assay is the incorporation of two labeling pulses with the nucleoside analogs CldU and IdU; Jackson \& Pombo, 1998. IdU and CldU incorporation into newly replicated DNA provides a mark that can be used for immunostaining at 


\section{MATERIALS AND METHODS}

a later stage. The following treated cells were incubated with CldU $50 \mu \mathrm{M}$ for 20 minutes, followed by IdU, both from Sigma-Aldrich $25 \mu \mathrm{M}$ for 60 minutes. After the labeling procedure, cells were harvested by washing them twice with ice-cold PBS followed by scraping them into $2 \mathrm{ml}$ cold PBS. Cell was centrifugated at $4^{\circ} \mathrm{C}$ and $5000 \mathrm{rpm}$ for 5 minutes and resuspended to a final concentration of 500,000 cells per milliliter in cold PBS. Labeled cells were spread onto Superfrost glass slides by applying a $5 \mu \mathrm{l}$ drop of cell suspension on top of the slide and airdrying for about 2 minutes. Cells were lysed by using DNA Fiber Spreading Buffer for 2 minutes at room temperature. The spreading step was carried by tilting the drop mixture of cells with lysis buffer slightly to run down slowly (about $3 \mathrm{~cm} / \mathrm{min}$ ) followed by air drying under a fume hood. Finally, the slides were fixed in a Fiber Assay Fixative solution for 10 minutes at room temperature before storage at $4^{\circ} \mathrm{C}$ for up to one month.

On the day of staining, fixed slides were rehydrated by incubating them with $\mathrm{ddH}_{2} \mathrm{O}$ once for 5 minutes and followed with acid treatment $(2.5 \mathrm{M} \mathrm{HCl})$ to denature the double-strand DNA and obtain single-stranded DNA. After denaturing the double stands DNA, the samples were washed twice with PBS (adjusted to $\mathrm{pH}$ 7.4). Before immunostaining, the slides were blocked with a $3 \%$ BSA in PBS solution to avoid unspecific antibody binding for 60 minutes. Primary antibodies that recognize CldU and IdU specifically were applied to dried slides in $250 \mu$ l blocking solution with a $1: 400$ dilution and incubated at $37^{\circ} \mathrm{C}$ for one hour. Samples were rinsed with PBS and fixed in a $4 \%$ formaldehyde solution for 10 minutes. The slides were again rinsed with PBS and incubated with a blocking solution three times for 5 minutes. Fluorescently labelled secondary antibodies AlexaFluor488 goat-anti-mouse and AlexaFluor555 goat-anti-rat recognized primary antibodies from rat origin and were applied to dried slides in 250 $\mu$ l with a dilution of 1:200 and incubated for 1.5 hours at room temperature. Final washing steps of rinsing once with PBS, washing twice with blocking solution for 5 minutes, rinsing with PBS and water were conducted before mounting with Vectashield mounting medium.

\section{xvii. Microscope analysis}

Each slide was analyzed with an Axio Scope A1 microscope (Zeiss) with filters for 488 and 555nm, an EC Plan-Neofluar 40x objective (Zeiss), and an Axio Cam MRc/503 camera (Zeiss). Ten to twenty images were taken from all parts of the slide and at least two slides per sample. 


\section{MATERIALS AND METHODS}

Measurements of fiber and label length, as well as their occurrence, was carried out using Image $\mathrm{J}$ and its cell counter plugin (Kurt de Vos, University of Sheffield, UK). All data were further processed using Microsoft Excel and GraphPad Prism.

\section{xviii. Statistical Analysis}

Statistical analysis was carried out using the GraphPad Prism Software (Versions 6 and 7). Analytical testing was performed using Graph Pad Prism 6 and 7. Mann-Whitney t-tests with two-tailed were calculated by Graph Pad Prism 6 and 7. 


\section{RESULTS}

\subsection{The depletion of centrosomal components not only reduces DNA replication fork progression but also induces DNA damage and activates the stress response}

To uncover whether centrosome integrity affects DNA replication, we sought to deplete several centrosome components using pooled siRNAs in H1299 cells. H1299 cells displayed a reduction in centrosome number upon depletion of several centrosome components (i.e., CEP192, CEP152, CCP110, and SAS6) (Figure 6.1 A-D) as well as a significant reduction in the corresponding mRNA levels (Figure 6.1 E). CEP192 localizes at the inner layer of the pericentriolar matrix, and it is crucial for the recruitment of pericentriolar material (PCM) (11). CEP152 is part of the outer PCM, and it is a critical protein for centrosome duplication through the recruitment of CDK2 to the site of centrosomes by forming a CEP152- CEP63-CDK5RAP2-WDR62 complex. It also acts as a scaffold protein that facilitates the interaction of PLK4 and CENPJ $(10,11)$. CCP110 is an additional centrosomal protein required for centrosome duplication but at a different stage of procentriole formation. CCP110 caps the mother centrioles and prevents cilia formation along with CEP97.

Additionally, CCP110 is required for correct spindle formation, regulating cytokinesis and, therefore, genomic stability. SAS6 is located at centrioles and is a centrosomal protein that plays a central role in the cartwheel assembly and ensuring the 9-fold symmetry. It is also necessary for centrosome duplication and biogenesis (14). Remarkably, each of these depletions significantly decreased fork progression in $\mathrm{H} 1299$ cells (Figure 6.1 F, G).

The induction of DNA damage in cells could be a reason behind the slower fork progression. Therefore, we intended to explore whether centrosome integrity can induce DNA damage; we firstly examined the accumulation of $\mathrm{yH} 2 \mathrm{AX}$ as a marker of DNA damage in cells with altered centrosome number. In addition to the observed reduction in centrosomes number as well as slowing down the DNA fork progression upon depletion of centrosomal components, we also captured the increased $\mathrm{yH} 2 \mathrm{AX}$ level (Figure 6.1 H, I). Searching for plausible activation of some stress-related markers, we managed to detect ATR/CHK1 phosphorylation (Figure 6.1 J). Thus, we conclude that the centrosome number is a determining factor for faithful DNA replication. 
RESULTS

A

B
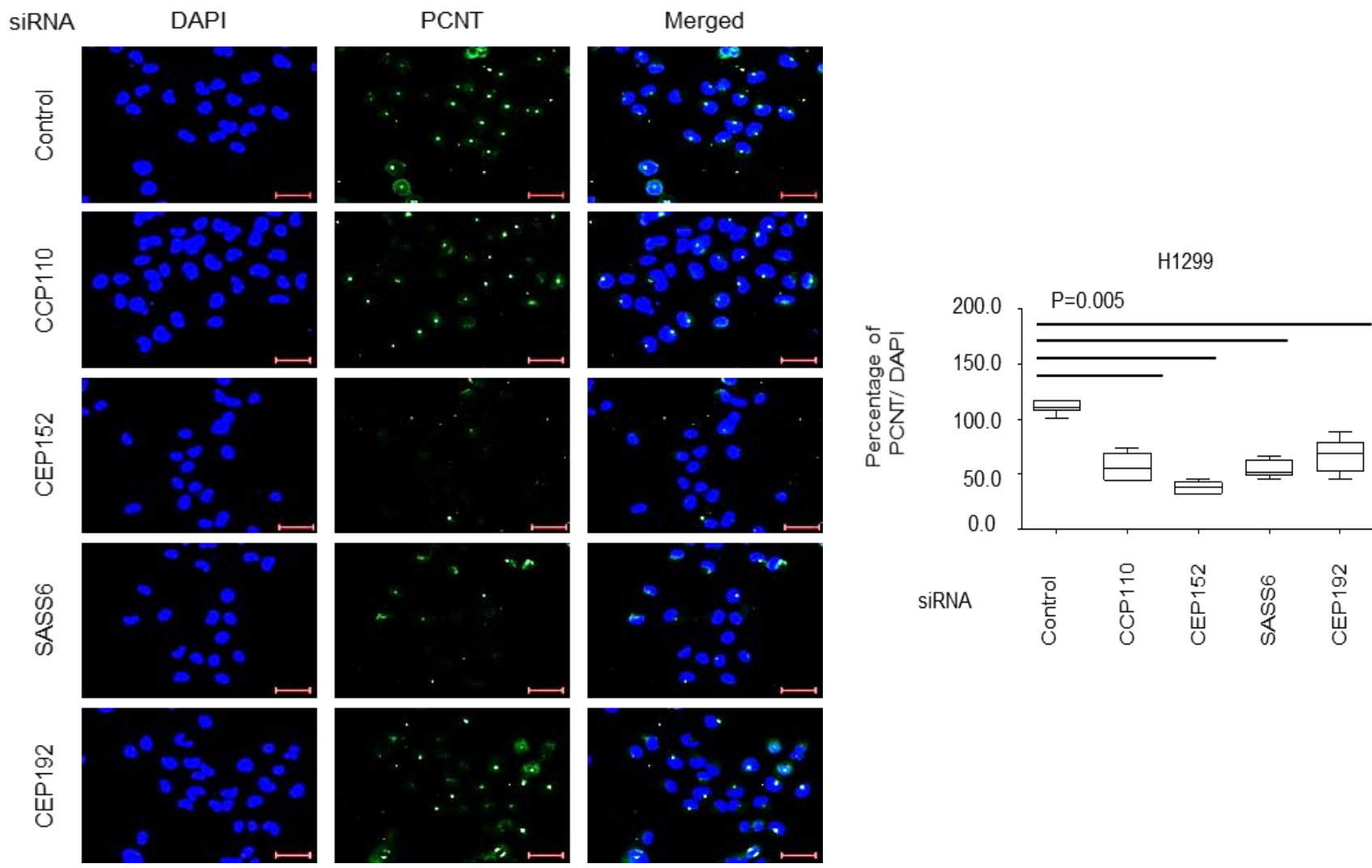

C

D
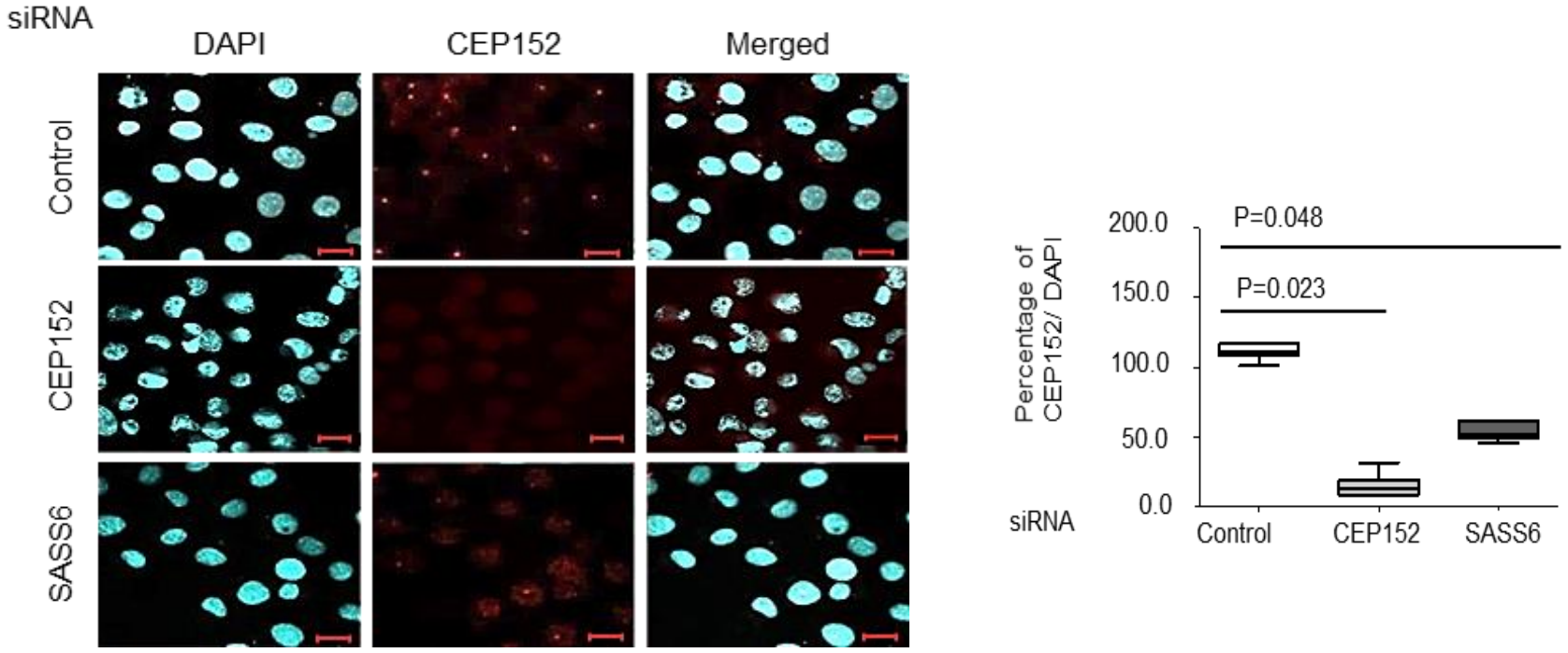
E

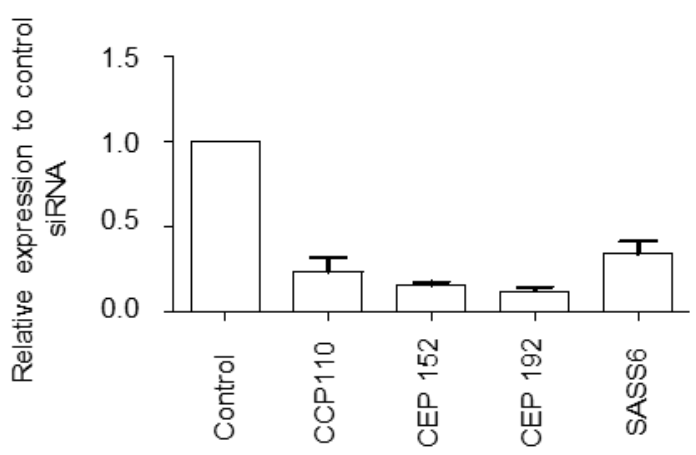

G
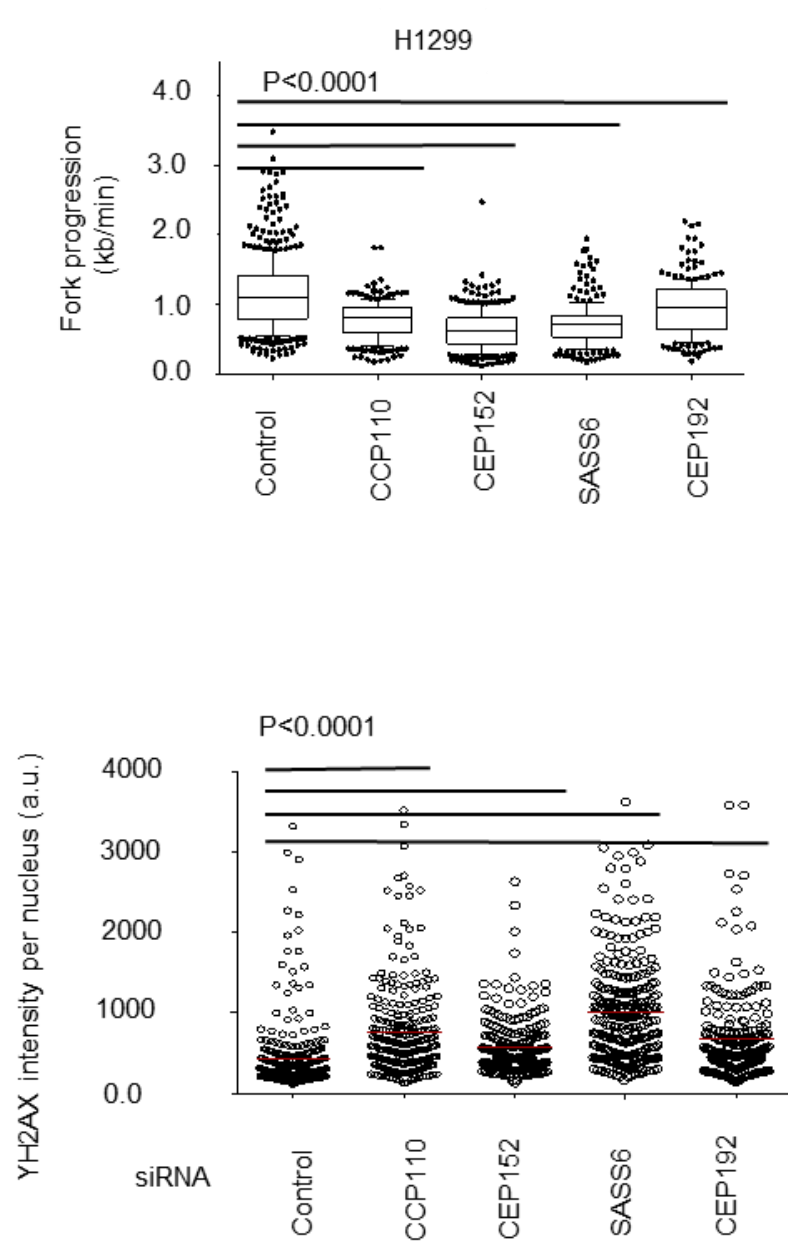

$\mathbf{F}$

Seeding

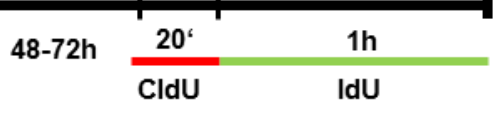

72h siRNA knockdown / 48hrs inhibitor

H

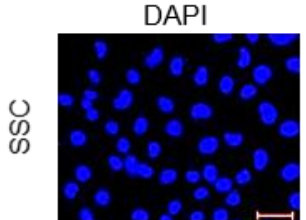

YH2AX
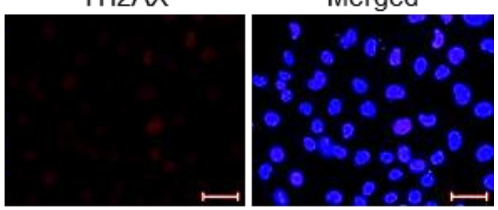

$\frac{\circ}{\frac{1}{0}}$
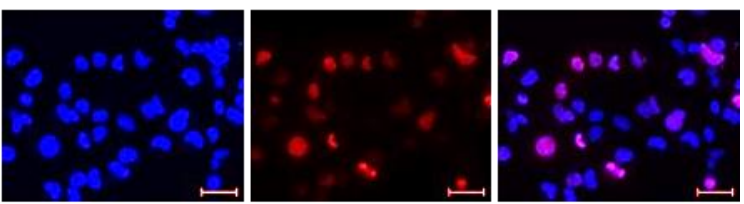

䒢
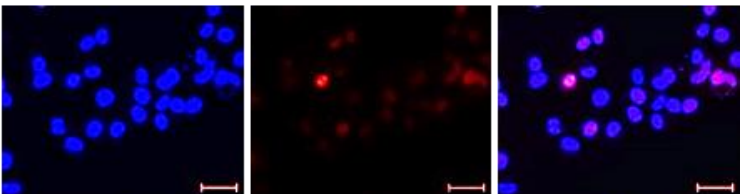

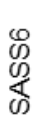
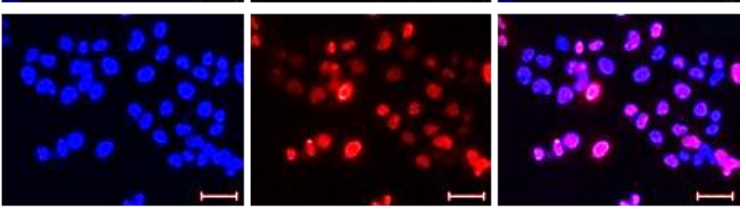

ळ
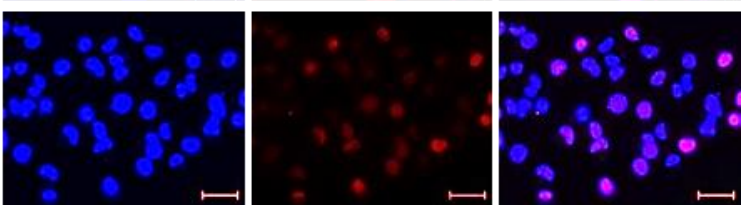


\section{RESULTS}

J

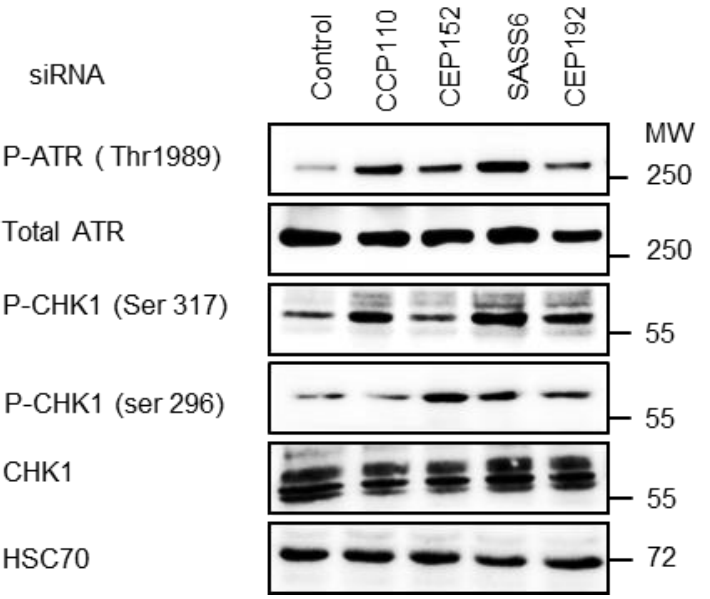

Figure 6.1: Depletion of centrosomal components interferes with DNA replication.

(A) Centrosome disintegration upon depletion of centrosomal components. H1299 cells were transfected with siRNAs against CEP152, SASS6, CCP110, and CEP192 for 72 hours. Centrosomes were detected by indirect immunostaining of PCNT, and 4',6-Diamidin-2phenylindol (DAPI) was used to outline the nuclei (scale bar $=20 \mu \mathrm{m}$ ).

(B) Quantification of the centrosome signals per cell to DAPI-stained nuclei from A. 300 cells from A was quantified per condition and presented as a percentage using GraphPad Prism. ${ }^{* * *} \mathrm{P}<0.0001$.

(C) Similar to A, cells were treated with CEP152, SASS6 siRNA for 72 hours. Centrosomes were detected using an antibody against CEP152 and 4',6-Diamidin-2-phenylindol (DAPI) was used to outline the nuclei (scale bar $=20 \mu \mathrm{m}$ ).

(D) Quantification of the centrosome signals per cell to DAPI-stained nuclei. One hundred fifty cells from C were quantified per condition and presented as percentage (the number of detectable centrosomes divided by the number of nuclei, multiplied by $100 \%$ ) using GraphPad Prism. ${ }^{\star \star * *} P<0.0001$. The results presented are an average of three biological replicates.

(E) The efficiency of single siRNA-mediated depletion of centrosomal components. RNA was isolated 72 hours post siRNA transfection (CCP110, CEP192, CEP152, and SASS6), followed by reverse transcription and quantitative polymerase chain reaction (qPCR). The relative expression upon knockdown displayed normalized to control siRNA \#1 and to the housekeeping gene HPRT1. Data represented is an average of $n=3$.

(F) Schematic workflow of cells treated with siRNA. H1299 cells were depleted of endogenous CEP152, CCP110, CEP192, or SASS6 by siRNA transfection for 72 hours and then labeled with CldU (20 min) and IdU (60 min).

(G) Compromised DNA replication fork progression upon centrosome depletion, as determined by DNA fiber assay. Cells were treated as in F followed by incubation with 5'-chloro-2'-deoxyuridine and iodo-deoxyuridine as indicated in panel I. Tracks of newly synthesized DNA were visualized by immunostaining of CldU (red) and IdU (green). Fork progression was determined through the length of the IdU label (kb/min). Two hundred fifty fibers were measured per condition per biological replicate and represented as a box plot.

(H) Accumulation of $\mathrm{yH} 2 \mathrm{AX}$ signal in cell depleting several centrosomal components. Cells were transfected with pool siRNA for 72 hours, followed by immunostaining against PCNT and the cell nuclei (DAPI) (scale bar $=20 \mu \mathrm{m}$ ).

(I) Quantification of the $\mathrm{yH} 2 \mathrm{AX}$ signal in cell nuclei upon centrosome depletion. The nuclear signal was quantified using the ImageJ software. The mean and distribution of three biological replicates (integrated) were calculated, and the significance was assessed using the MannWhitney test.

(J) Activation of ATR/CHK1 upon centrosome depletion. Lysates of H1299 cells were prepared 72 hours after depleting centrosomal components with siRNA as in (A), followed by immunoblot analysis. 


\section{RESULTS}

\subsection{Inhibiting PLK4 activity reduces cell proliferation, impairs DNA replication fork progression, and activates the stress response}

Polo-like Kinase 4 (PLK4) is another centrosomal protein that is being recruited to the site of centrosomes during the late G1-S phase by the scaffold protein CEP152. PLK4 is essential in the initiation step of centrosome duplication. A few inhibitors were developed to target this protein, which tends to be overexpressed in several cancer types, among which the small compound Centrinone B (108). Treating H1299 cells with 500 nM Centrinone B for 48 hours not only led to a substantial reduction in the number of detectable centrosomes (Figure 6.2 A-D), as described (108) but also impaired the progression of DNA replication forks (Figure 6.2 E, F). Moreover, we observed a global reduction of EdU incorporation (Figure $6.2 \mathrm{G}, \mathrm{H}$ ), indicative of replication stress, as well as a reduction in the cell proliferation rate (Figure $6.2 \mathrm{I}$ ).

To assess DNA replication in this context, we performed fiber assays and measured the progression of single replication forks using several cell types. $\mathrm{H} 1299$ cells not only exhibited a substantially decreased fork progression in a highly significant manner, but also SW48 cells treated with Centrinone B displayed a reduction in the fork progression in a similar way to H1299 cells (Figure 6.2 J). Impairment of centrosomes appears to extend its impact to non-transformed cells. Our fiber assay shows the reduction in fork progression upon PLK4 inhibition in retinal pigment epithelial cells (RPEs) that were immortalized (but not transformed) by hTert (Figure $6.2 \mathrm{~K}$ ).

Moreover, it seems like the p53 status does not further compromise DNA replication and cell proliferation upon PLK4 inhibition. Fiber assay using HCT116 cell lines that do or do not contain functional copies of the TP53 gene showed a reduction in fork progression upon PLK4 inhibition as well as a decrease in centrosome numbers (Figure 6.2 L-N). Using a Celigo Imaging Cytometer, we observed a slowdown in cell proliferation as a consequence of PLK4 inhibition in H1299 as well as HCT116 cells (Figure $6.2 \mathrm{O}, \mathrm{P}$ ). Additionally, we explored the possibility of induction of DNA damage under these conditions by assessing the accumulation of $\mathrm{yH} 2 \mathrm{AX}$ as a marker of DNA damage in cells with impaired centrosome number. H1299 cells treated with Centrinone B showed an increase in yH2AX levels (Figure 6.2 Q, R). The observed accumulation of $y \mathrm{H} 2 \mathrm{AX}$ seems to be a result of the activation of the stress response pathway ATR/CHK1 (Figure 6.2 S). Thus, we conclude that PLK4 activity is indeed required to achieve accurate DNA replication. 
RESULTS

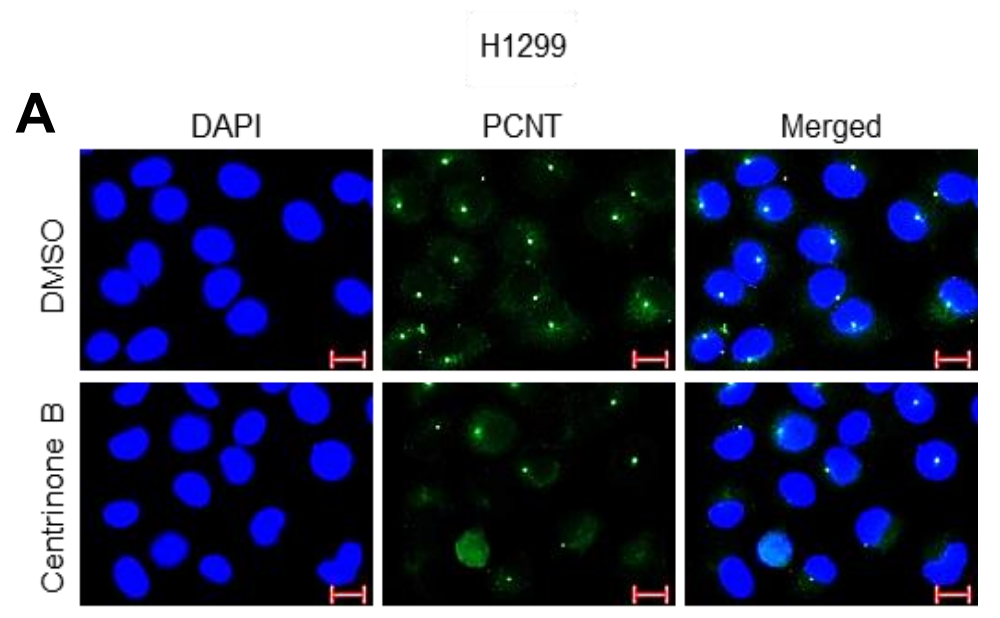

B

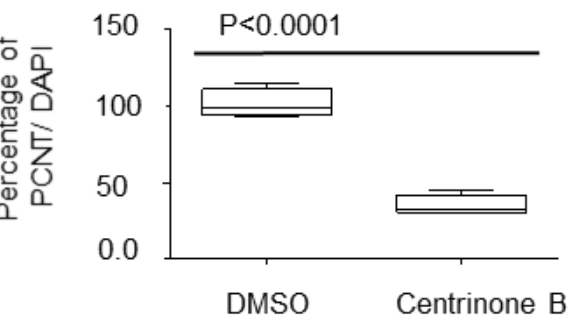

C
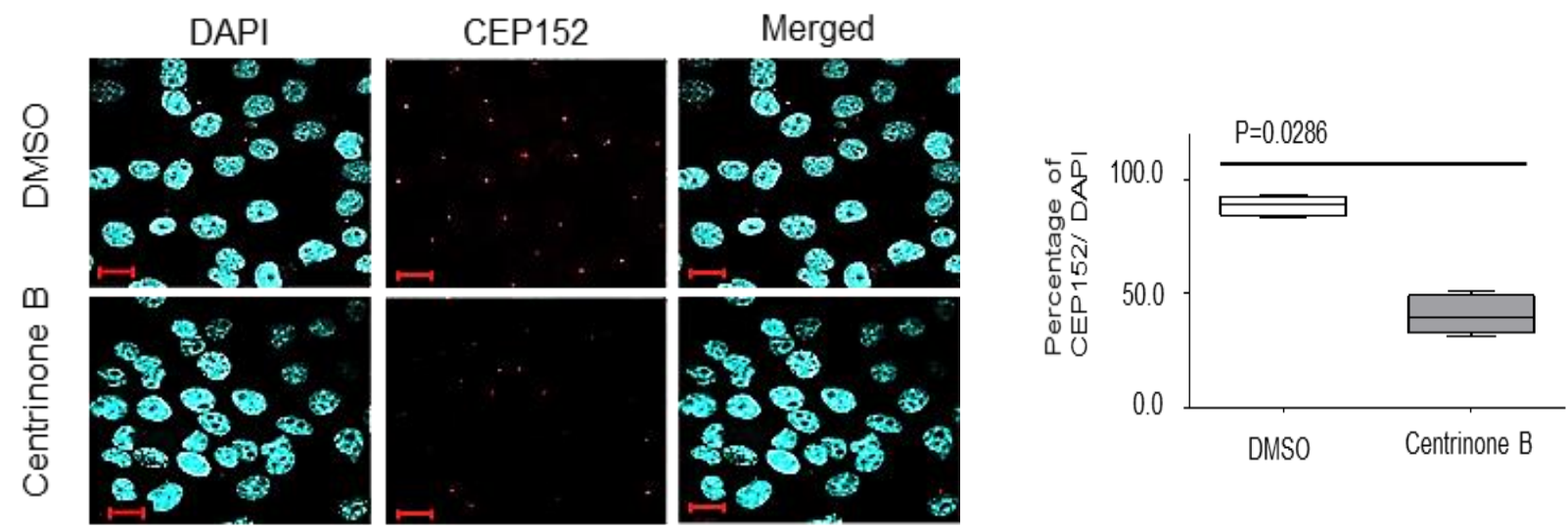

E

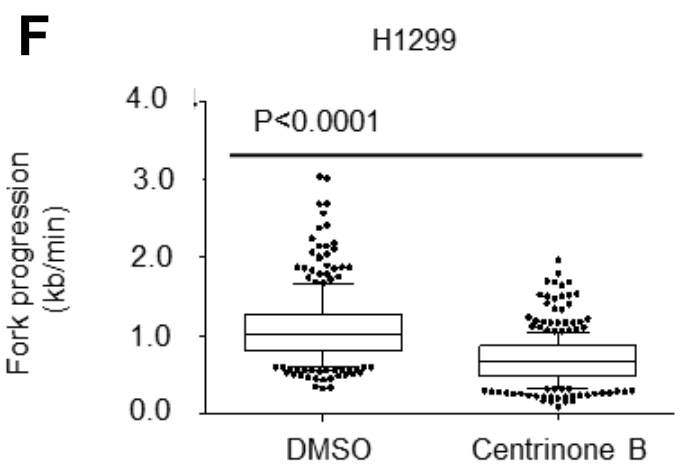


RESULTS

G

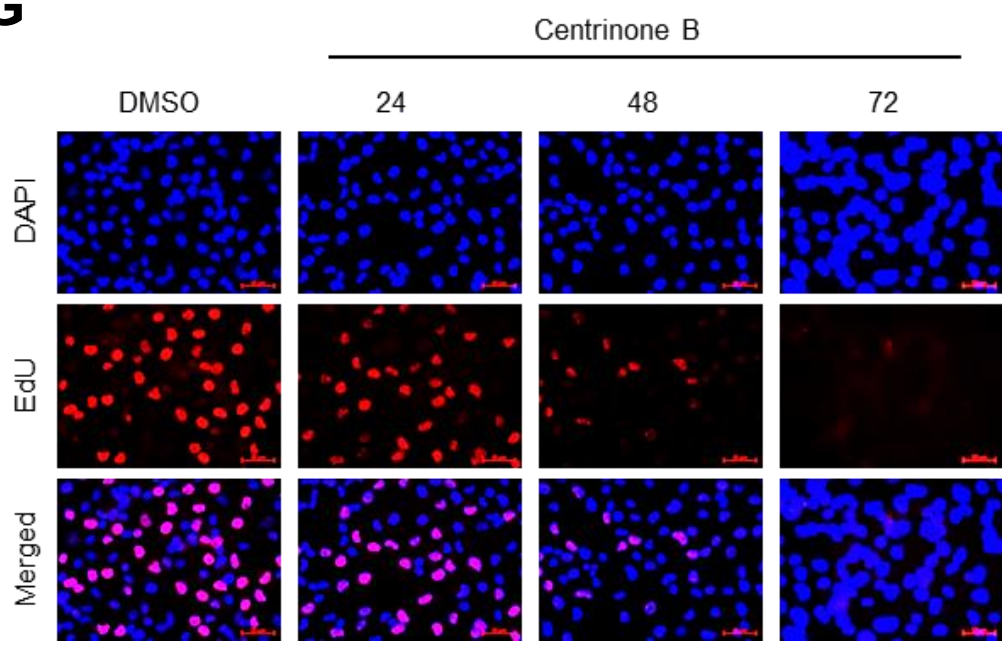

H

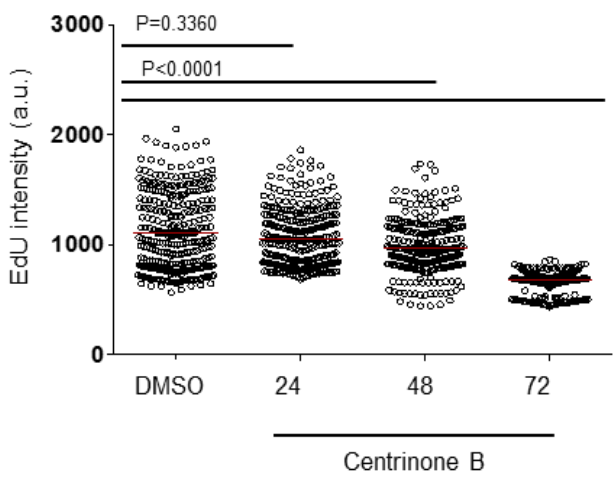

\section{H1299}

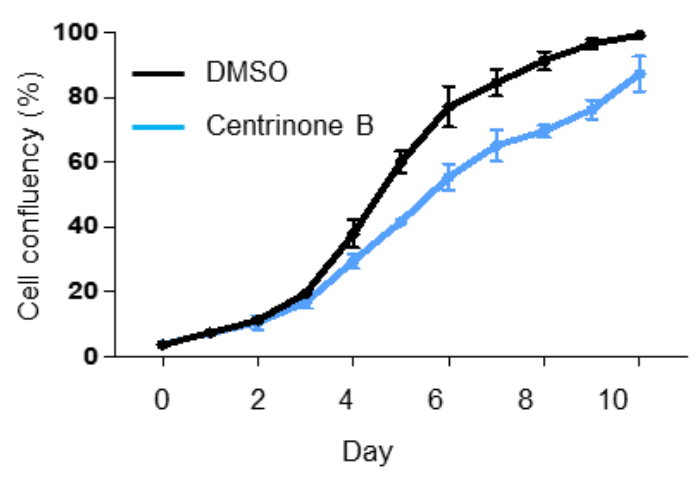

K

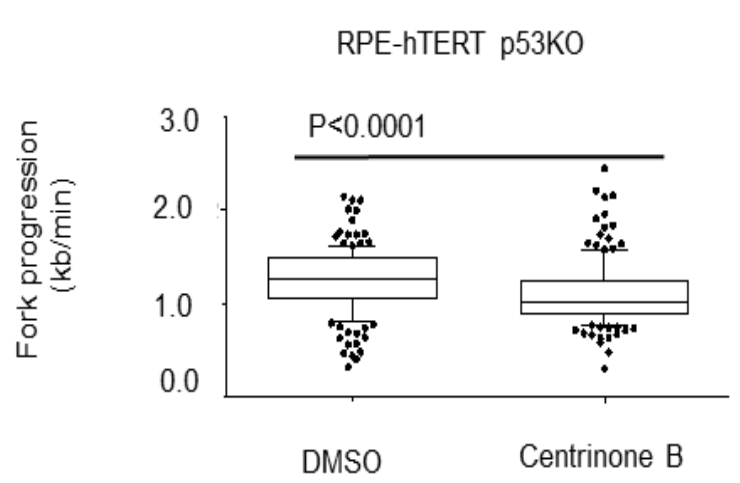

J

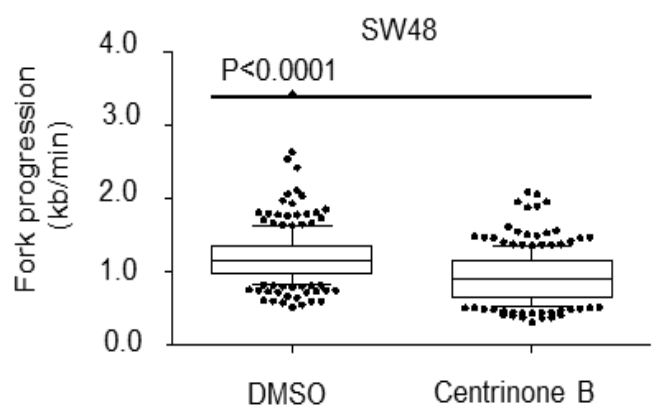

L

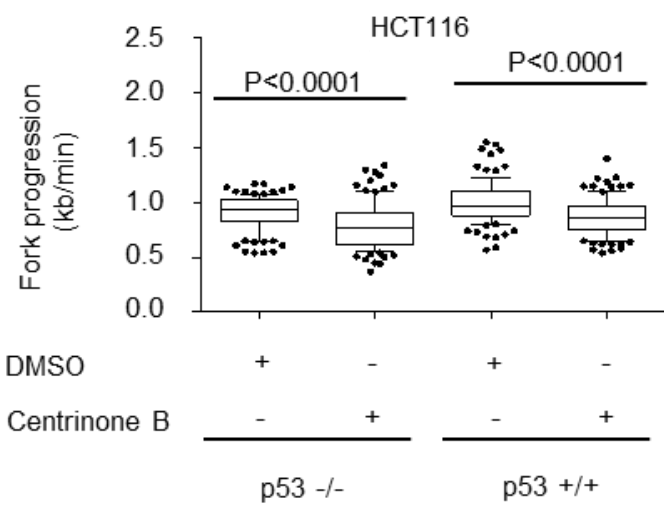


M

HCT116 p53 -/-
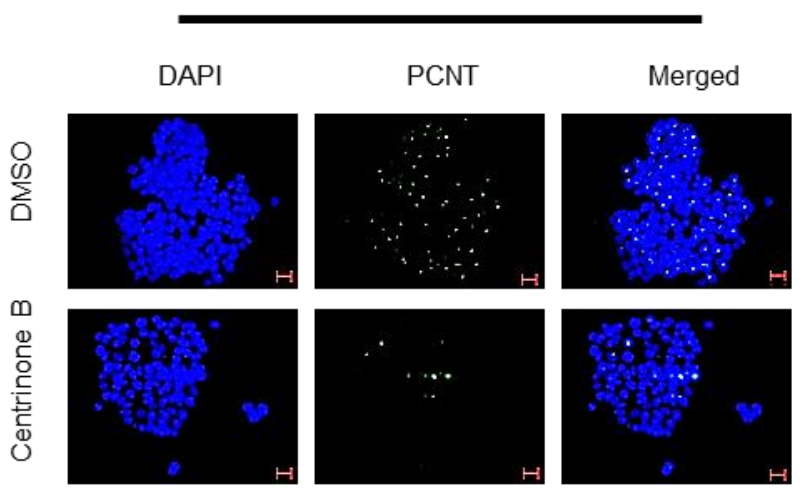

$\mathbf{N}$

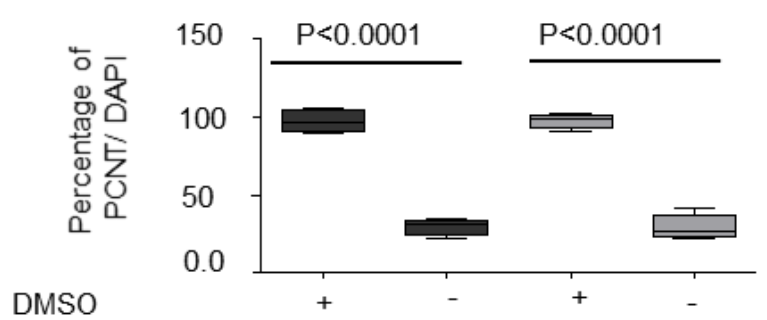

Centrinone B

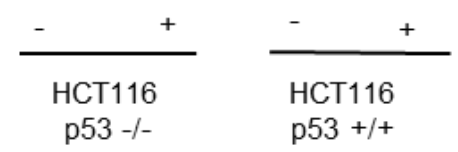

P

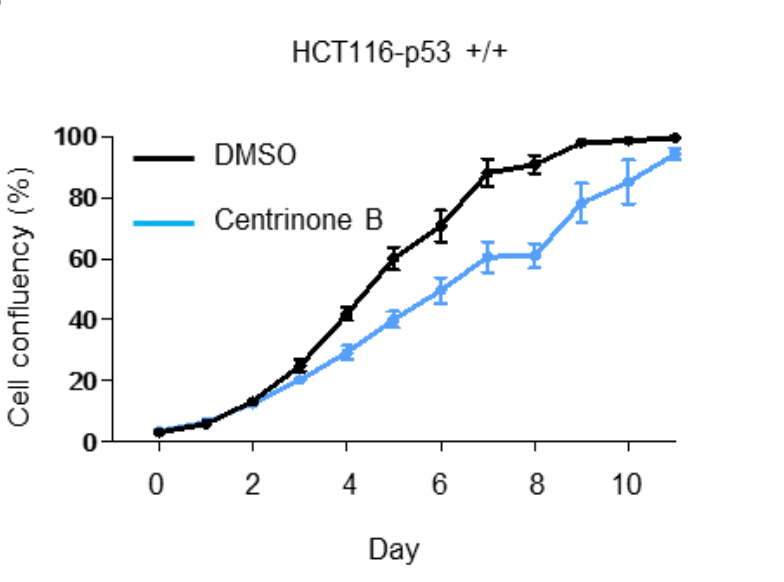

HCT116 p53 +/+

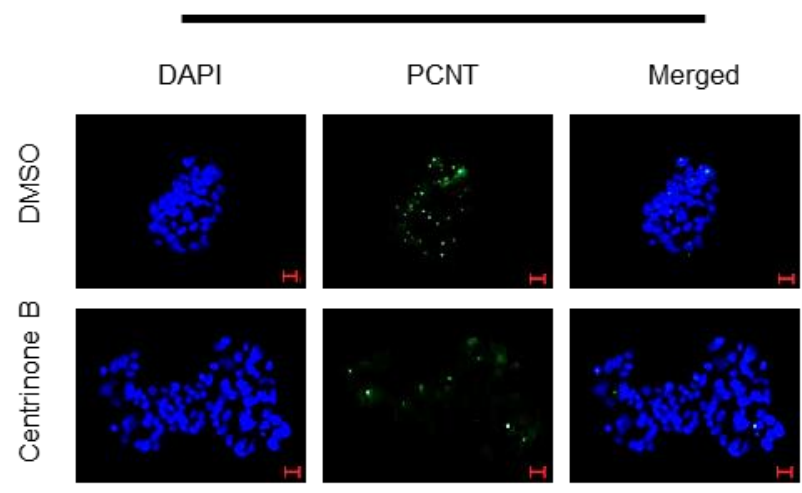

0

HCT116-P53 -/-

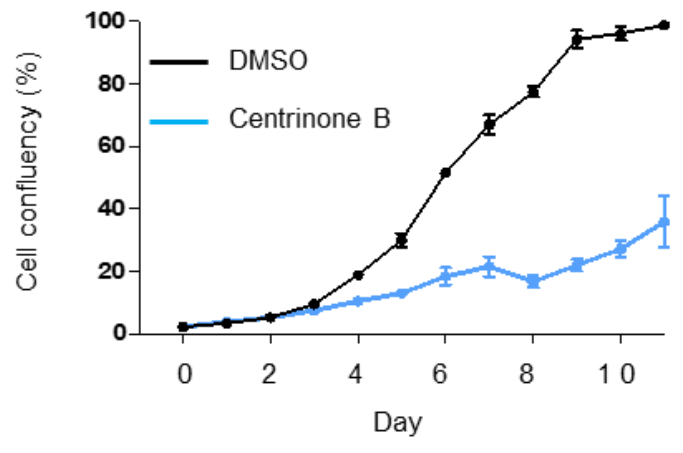

$\mathbf{Q}$

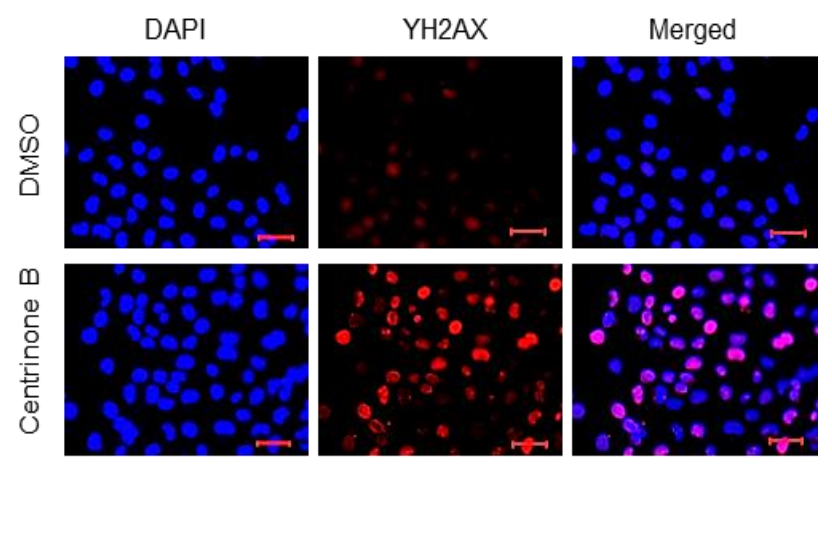




\section{RESULTS}

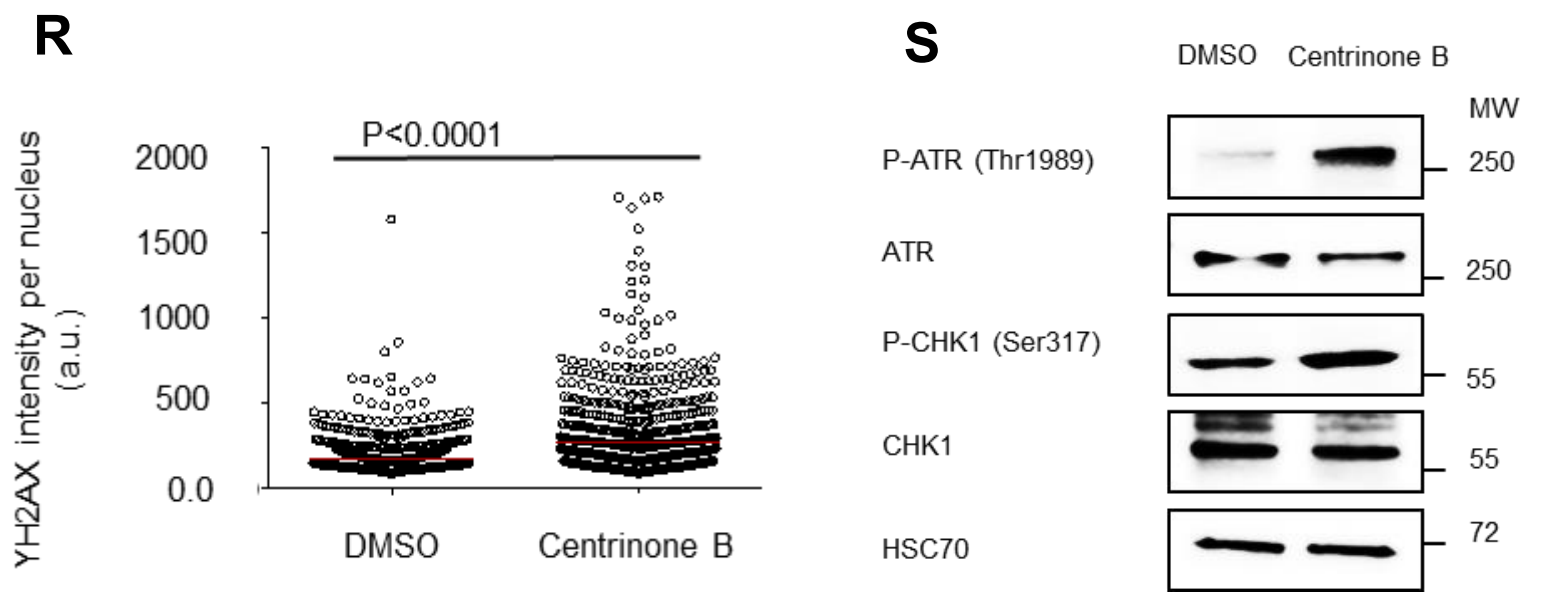

Figure 6.2: Inhibition of the centrosomal component PLK4 interferes with DNA replication.

(A) Detection of centrosome disintegration upon PLK4 inhibition. Cells were treated with $500 \mathrm{nM}$ Centrinone B for 48 hours. Centrosomes (immunostaining of PCNT) and the cell nuclei (DAPI) were detected (scale bar $=20 \mu \mathrm{m}$ ).

(B) Quantification of the centrosome signals per cell to DAPI-stained nuclei. Upon centrosomes depletion, 300 cells were quantified per condition and presented as percentage (the number of detectable centrosomes divided by the number of nuclei, multiplied by $100 \%$ ) using GraphPad Prism. ( $\mathrm{n}=3),{ }^{* * \star *} \mathrm{P}<0.0001$.

(C) Detection of centrosome disintegration upon PLK4 inhibition. Cells were treated with $500 \mathrm{nM}$ Centrinone B for 48 hours. Centrosomes (immunostaining of CEP152) and the cell nuclei (DAPI) were detected (scale bar $=20 \mu \mathrm{m}$ ).

(D) Quantification of the centrosome signals per cell to DAPI-stained nuclei. From C, 200 cells upon centrosomes depletion were quantified per condition and presented as percentage (the number of detectable centrosomes divided by the number of nuclei, multiplied by $100 \%$ ) using GraphPad Prism. $(n=3),{ }^{* * \star} P<0.0001$.

(E) Schematic workflow of cells treated with Centrinone B. H1299 cells was treated with 500 nM Centrinone B for 48 hours and then labeled with CldU (20 min) and IdU (60 min).

(F) DNA fiber assays detected reduced DNA replication fork progression in response to PLK4 inhibition in H1299 cells. Cells were treated as described in (A). Cells were treated with $500 \mathrm{nM}$ Centrinone B for 48 hours, followed by incubation with 5'-chloro-2'-deoxyuridine and iodo-deoxyuridine as indicated in panel I. Tracks of newly synthesized DNA were visualized by immunostaining of CldU (red) and IdU (green). Fork progression was determined through the length of the IdU label (kb/min). Two hundred fifty fibers were measured per condition per biological replicate and represented as a box plot.

(G) Representative images for the EdU incorporation signal in synchronized H1299 cells treated with 500 nM Centrinone B for 24-72 hours. H1299 cells were treated with Centrinone B for 24-72 hours, and EdU incorporation was measured by click-it.

(H) Quantification of images from (G). The signal was quantified using the ImageJ software. The mean and distribution of three biological replicates (integrated) was calculated, and the Mann-Whitney t-test assessed the significance.

(I) Reduction in cell proliferation upon PLK4 inhibition with Centrinone B. 5*103 H1299 cells was seeded in each well of a $24-$ well plate. Cells were treated with DMSO or $300 \mathrm{nM}$ Centrinone B. Cell proliferation capacity was measured using the CeligoTM Cytometer (Nexcelom, software version 2.0). Confluence was measured every 48 hours for ten days. The experiment was carried out in three biological replicates and six technical replicates for each time point. Note that most error bars are too narrow to be displayed.

(J) Reduction in fork progression in SW48 cells after PLK4 inhibition. Cells were treated as described in F, followed by fiber assay. Two hundred fibers were measured per condition per biological replicate and represented as a box plot. 


\section{RESULTS}

(K) Impact of PLK4 inhibition on non-transformed RPE-hTert cells. Cells were treated as described in F, followed by fiber assay. One hundred fifty fibers were measured per condition per biological replicate and represented as a box plot.

(L) TP53 does not further compromise the fork progression in cells with impaired centrosomes. HCT116 cells that do or do not contain functional P53 gene were treated with $500 \mathrm{nM}$ Centrinone B for 48 hours, then subjected to fiber assay. One hundred fifty fibers were measured per condition.

(M) Detection of centrosome disintegration upon PLK4 inhibition. HCT116 cells deficient and proficient in P53 were treated with $500 \mathrm{nM}$ Centrinone B for 48 hours. Centrosomes (immunostaining of PCNT) and the cell nuclei (DAPI) were detected (scale bar $=20 \mu \mathrm{m}$ ).

(N) Quantification of centrosomes from I. Quantification of the centrosome signals per cell to DAPI-stained nuclei. One hundred fifty cells from $A$ were quantified per condition and presented as percentage (the number of detectable centrosomes divided by the number of nuclei, multiplied by $100 \%$ ) using GraphPad Prism. $(n=3),{ }^{* * *} P<0.0001$.

(0) Inhibition of cell proliferation in HCT116-P53-/- upon PLK4 inhibition with Centrinone B. 5*10 ${ }^{3}$ in HCT116-P53-/- cells were seeded in each well of a 24-well plate. Cells were treated with DMSO or $300 \mathrm{nM}$ Centrinone B. The experiment was carried out as described in L.

(P) Cell proliferation inhibition in HCT116-P53+/+ upon PLK4 inhibition with Centrinone B. 5*103 in HCT116-P53+/+ cells were seeded in each well of a 24-well plate. Cells were treated with DMSO or $300 \mathrm{nM}$ Centrinone B. The experiment was carried out as described in L.

(Q) Accumulation of $\mathrm{yH} 2 \mathrm{AX}$ signal in cells treated with PLK4 inhibitor. Cells were treated with $500 \mathrm{nM}$ of Centrinone B for 48 hours, followed by immunostaining for PCNT and the cell nuclei (DAPI) (scale bar $=20 \mu \mathrm{m}$ ).

(R) Quantification of the $\mathrm{yH} 2 \mathrm{AX}$ signal in cell nuclei upon treatment with Centrinone B for 48 hours from A. The nuclear signal was quantified using the ImageJ software. The mean and distribution of three biological replicates (integrated) were calculated, and the Mann-Whitney test assessed the significance.

(S) Activation of ATR and CHK1 upon PLK4 inhibition. H1299 cells were treated as described in Q, briefly with $500 \mathrm{nM}$ Centrinone B for 48 hours, then subjected to immunoblot analysis. 


\section{RESULTS}

\subsection{PLK4 inhibition drives cells towards polyploidy}

Polyploidy is another source of DNA replication stress. It is known that centrosome abnormalities correlate with both numerical and structural chromosomal instability (CIN). Both high and insufficient levels of PLK4 are associated with abnormal spindle poles, uneven distribution of chromosomes, and numerical chromosomal abnormalities (4). However, it is still unknown to what extent inhibiting PLK4 using Centrinone $B$ has a direct impact on the spindle and to what extent DNA replication stress is involved. Therefore, we investigated the effect of long-term treatment with Centrinone B on the cell cycle as well as on chromosomal numbers. Interestingly, cell cycle and the chromosomal number remain the same after 48 hours, and only after seven days post-treatment an effect could be seen on both cell cycle as well as chromosomal number (Figure 6.3 A-C). In summary, our results suggest that DNA replicative stress is the immediate response to centrosomal depletion and that accumulation of replicative stress but not only could drive the cells towards aneuploidy and genomic instability.

A

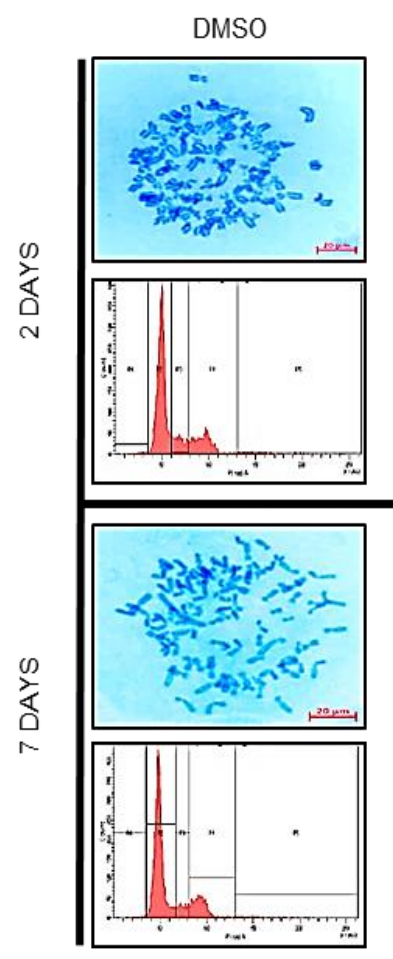

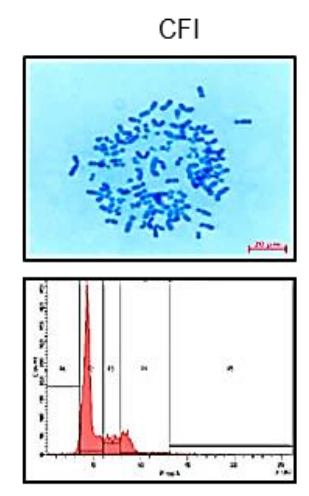
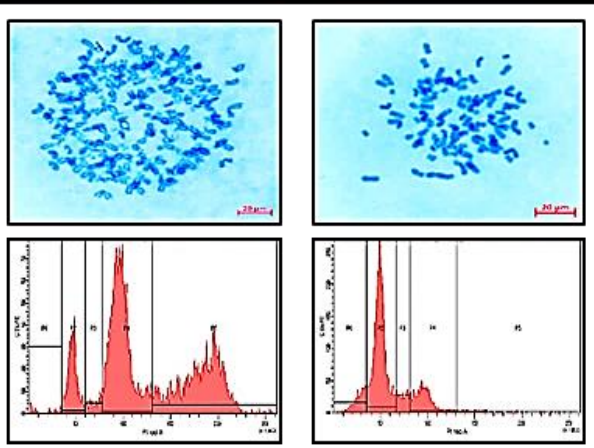

B

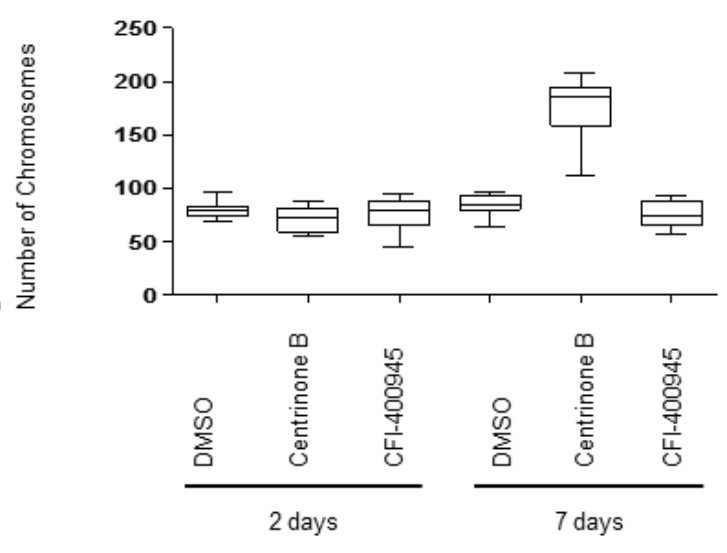




\section{RESULTS}

\section{C}
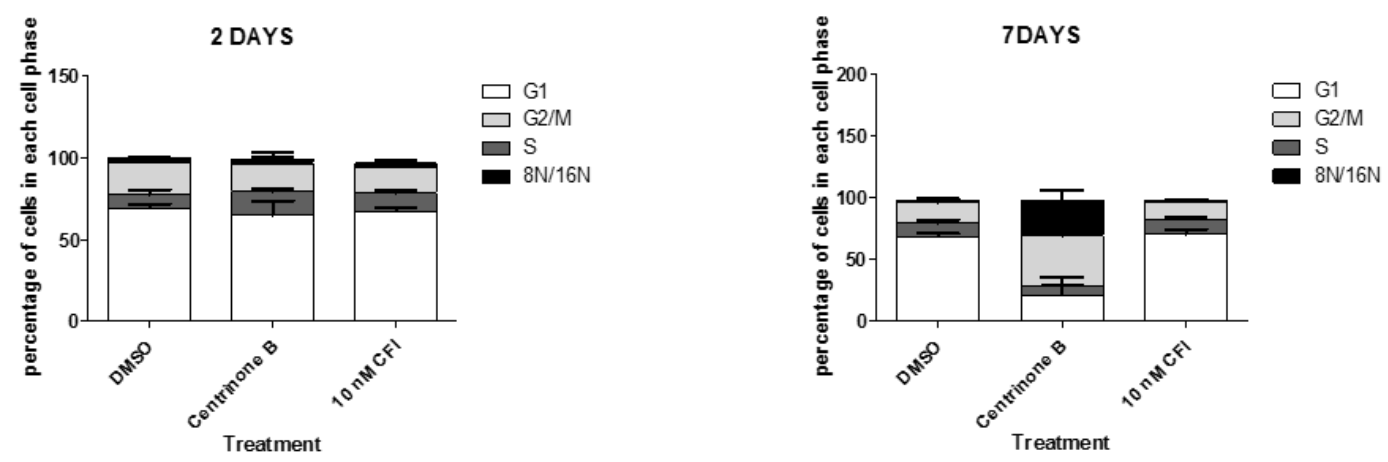

Figure 6.3: PLK4 inhibition leads to polyploidy after release.

(A) Upper panel: Representative images of chromosome sets from three biological replicates. Cells were treated with $500 \mathrm{nM}$ of Centrinone $\mathrm{B}$ or $10 \mathrm{nM}$ of CFI-400945 for two to seven days, followed by spreading and visualizing chromosomes as indicated in material and methods. Chromosomes were stained with $8 \%$ Giemsa solution, and images were acquired by microscopy (100x, bright field mode). Lower panel: representative images of the cell cycle profile corresponding to the chromosomal spreading experiment. DNA content and thus, cell cycle distribution were assessed by propidium iodide (PI) staining and flow cytometry.

(B) The number of chromosomes per cell was counted manually from $A$ and plotted using GraphPad Prism ( $n=3$ cells per time point and condition).

(C) Quantification of cell cycle profile from A. Data is represented as an average of three biological replicats 


\subsection{Impairment of centrosomes causes replication stress independent of mitosis}

The results suggested that centrosomal integrity is required to maintain the processivity of DNA replication. However, it was not clear yet whether the observed effect on DNA progression is direct, or whether centrosome disruption first impairs chromosome segregation during mitosis, which might then reduce fork replication during the next $S$ phase. The latter scenario was plausible for two reasons. Firstly, centrosome disruption indeed impairs the function of the mitotic spindle and thus chromosome segregation (121). Moreover, even one additional chromosome (numerical aneuploidy) is sufficient to trigger DNA replication stress (113). Therefore, we developed a strategy of disrupting centrosomes and assessing DNA replication without allowing the cells to go through mitosis during the time of centrosome impairment. The technical difficulty in doing so consisted of the prolongation of the period required to deplete centrosomal components - a minimum of 72 hours for siRNA knockdown or 48 hours for PLK4 inhibition. Therefore, we sought to arrest the cells in G1 for 48 hours to disrupt the centrosome during this time. Only after that, the cells were released to $S$ phase but not allowed to reach mitosis. To do so, we first arrested the cells in G1, using the cyclin-dependent kinase 4 (CDK4) inhibitor Palbociclib (122). As shown in (Figure 6.4 A), this was achieved in less than 24 hours. Washing off Palbociclib allowed the cells to re-enter the cell cycle, but with variable time frames required for entering the $S$ phase. To synchronize this entry, we released the cells from the CDK4 inhibitor but at the same time, added thymidine, which is known to block the cell cycle right after entry into the S phase (123). We then released the cells from the thymidine block for three hours and thereby synchronizing the cells in the $S$ phase (Figure 6.4 A). By doing this, we were able to disrupt the composition of centrosomes and analyze DNA replication without entering mitosis. Using this system (Figure 6.4 A), we still observed diminished DNA replication fork progression upon PLK4 inhibition in both Centrinone B and CFI-400945 (another potent PLK4 inhibitor) treated cells (Figure 6.4 B, E). A substantial decrease in the overall incorporated EdU was also observed after PLK4 inhibition in synchronized cells (Figure 6.4 C, D), which indicates global replicative stress captured in these cells.

Furthermore, PLK4-depleted cells experienced the same effect on the fork progression (Figure 6.4 F, G). Likewise, the depletion of centrosomal components also led to a significant reduction in the fork progression (Figure 6.4 H). Thus, the disruption of centrosomal composition interferes with the processivity of DNA replication independently of chromosome missegregation. 
A

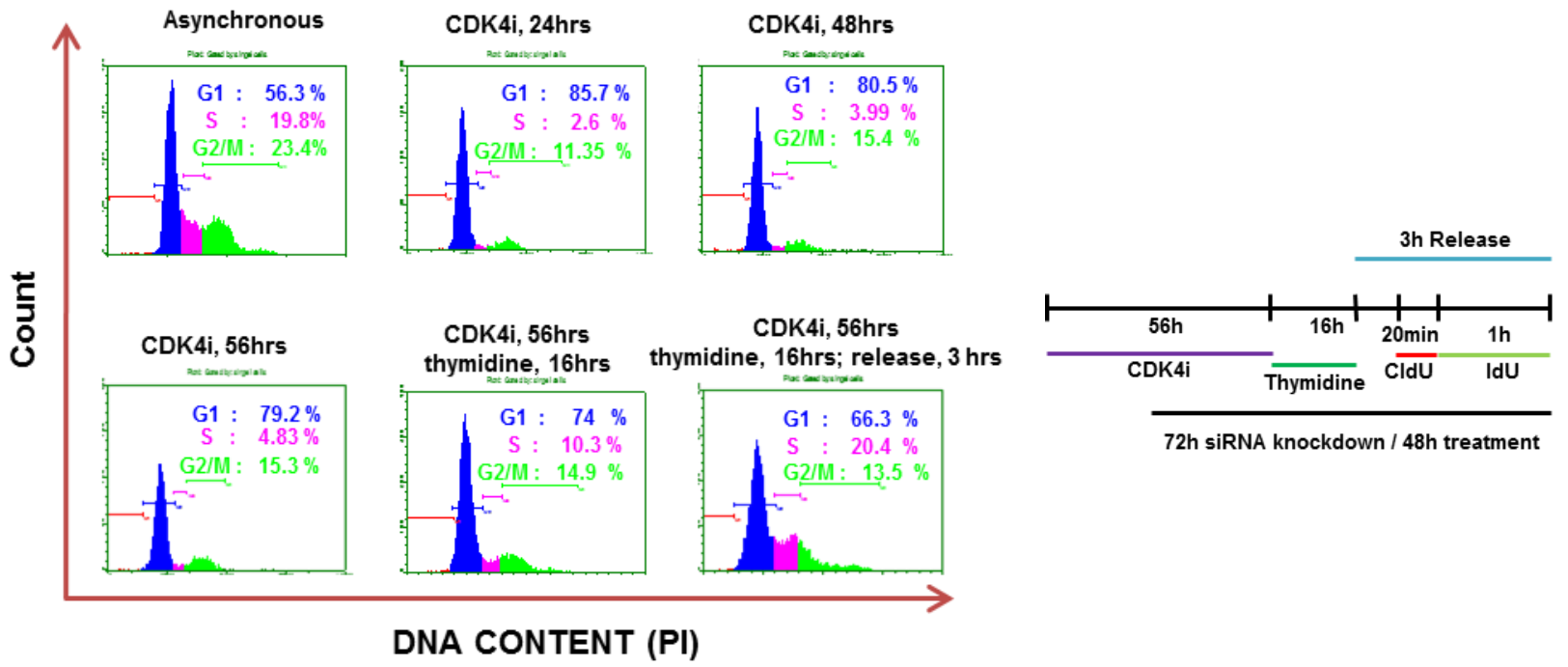

B

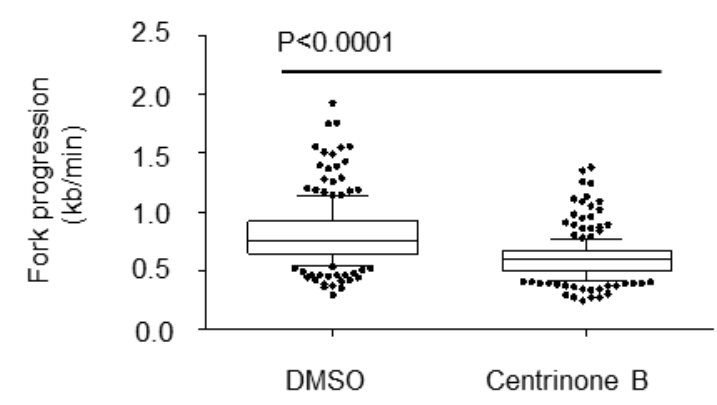

D

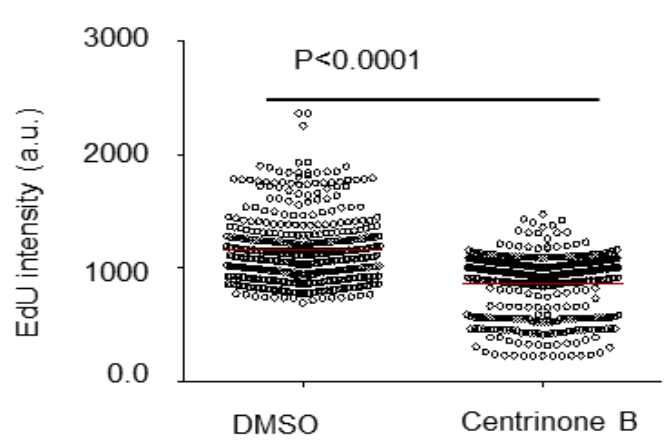

C

DMSO Centrinone B
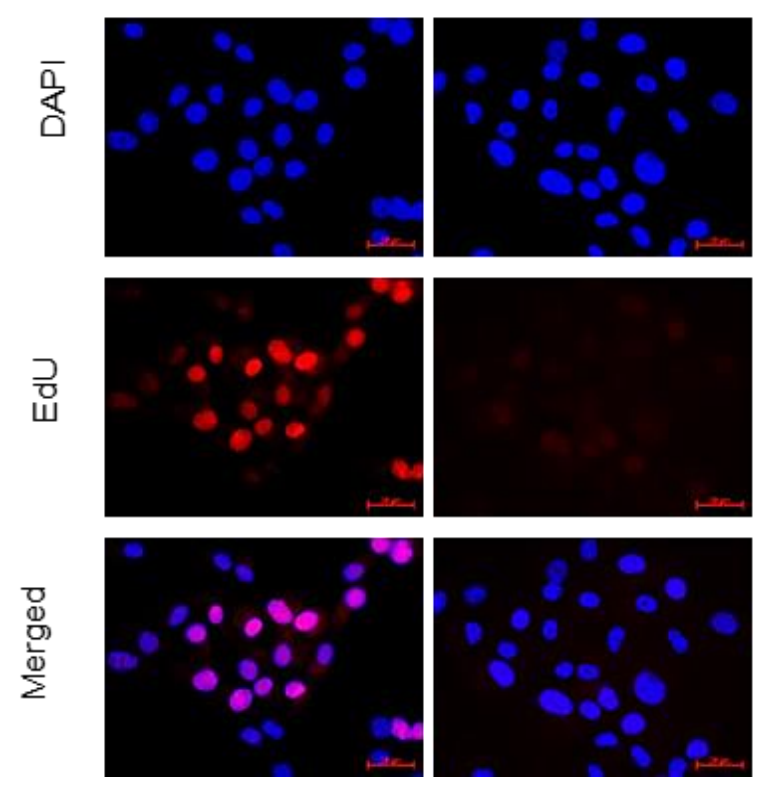
E

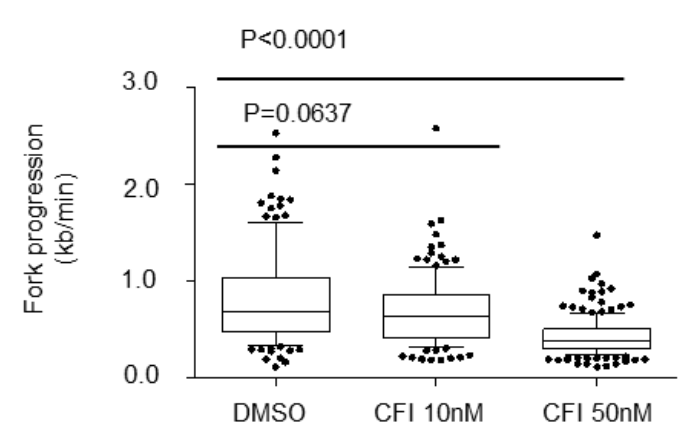

G

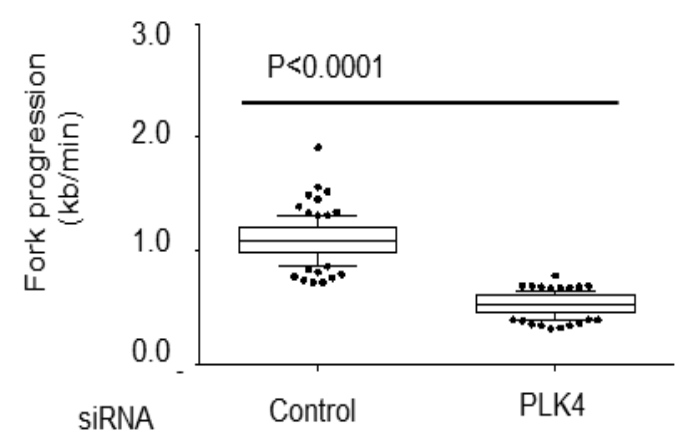

F

SiRNA

PLK4

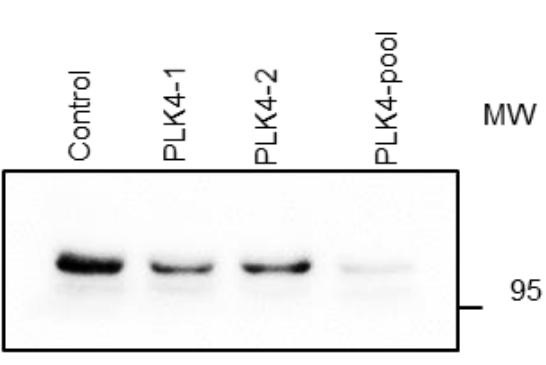

GAPDH

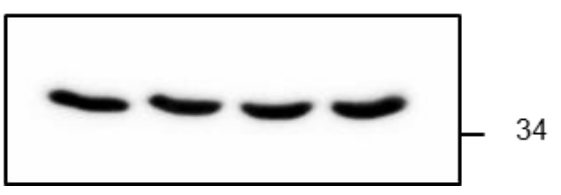

H

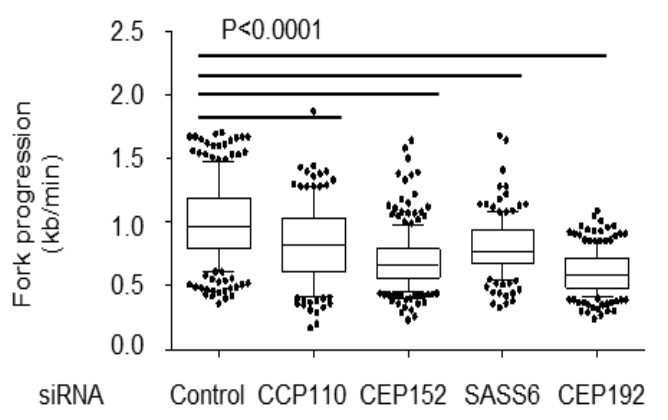

Figure 6.4: Impairment of centrosomes causes replication stress independent of mitosis.

(A) Prolonged G1 arrest and synchronized entry into the S phase. H1299 cells were treated with $5 \mu$ M CDK4 inhibitor (PD 0332991, also known as Palbociclib) for 24,48 , or 56 hours. Cells treated with the CDK4 inhibitor for 56 hours were subsequently incubated with $2 \mathrm{mM}$ thymidine for 16 hours. Afterward, the cells were washed and released into $S$ phase for 3-4 hours. DNA content and thus, cell cycle distribution were assessed by propidium iodide (PI) staining and flow cytometry. Schematic workflow of cell synchronization with CDK4 inhibitor $(5 \mu \mathrm{M})$ and thymidine $(2 \mathrm{mM})$.

(B) Cells were synchronized as outlined in (A) and incubated with 5'-chloro-2'-deoxyuridine, followed by iodo-deoxyuridine as indicated. Tracks of newly synthesized DNA were visualized by immunostaining of CldU (red) and IdU (green). Fork progression was determined through the length of the IdU label ( $\mathrm{kb} / \mathrm{min})$. Two hundred fibers were measured per condition and represented as a boxplot. During the initial G1 arrest phase, PLK4 was inhibited throughout the experiment (including the initial G1 arrest phase) by Centrinone B (500 nM).

(C) EdU incorporation of synchronized $\mathrm{H} 1299$ cells upon Centrinone B treatment. Cells were treated with $500 \mathrm{nM}$ for 48 hours. Two hours before harvesting, the cells were incubated with $10 \mu \mathrm{M}$ of EdU. The staining of the incorporated EdU was carried out using Click-iT, as described in the Material and Methods section.

(D) Quantification for the global reduction in the EdU incorporation upon Centrinone B treatment. Synchronized H1299 cells from C were treated with $500 \mathrm{nM}$ for 48 hours. Two hours before harvesting, cells were incubated with $10 \mu \mathrm{M}$ of EdU. Staining the incorporated EdU was carried out using Click-iT as described in the material and methods.

(E) Cells were analyzed as described in (B) after the treatment with $10 \mathrm{nM}, 50 \mathrm{nM}$ of CFI-400945, another potent PLK4 inhibitor.

(F) Validation of the PLK4 siRNA efficiency using western blot as single siRNA and pooled. $10 \mu \mathrm{M}$ of pool siRNA was used to deplete PLK4 in $\mathrm{H} 1299$ cells for 72 hours.

(G) Analyses as in (B) but after PLK4 knockdown.

(H) Cells analyzed as in (B), after centrosomes depletion using several targets of pooled siRNA for 72 hours. 


\subsection{Centrosomal disintegration induces $\mathrm{p} 38 / \mathrm{MK} 2$ signaling, and this is required for replication stress}

Investigating a potential mechanism, which is responsible for our previous observations on slowing the fork progression upon centrosomes impairments, we examined the activity of p38/MK2 signaling, a pathway that was previously shown to be necessary for reducing DNA replication by nucleoside analogs or CHK1 inhibition $(114,124)$. Indeed, the phosphorylated and thus active forms of p38 and MK2 were strongly enhanced upon PLK4 inhibition (Figure 6.5 A), and the same was found for the bona fide MK2 substrate Hsp27 (125). Similar findings were reported previously, albeit in non-synchronized cells $(126,127)$. As we ruled out the possibility to have this effect due to chromosomal aberration, the disruption of centrosomes activates p38/MK2 signaling, independent of mitotic dysfunction.

Next, we examined whether the activation of p38/MK2 signaling is a cause of the impaired DNA replication upon centrosome disintegration. We treated the cells with the PLK4 inhibitor Centrinone $\mathrm{B}$. While assessing DNA replication using fiber assays, we incubated the cells with a pharmacological inhibitor of MK2 (128). And indeed, DNA replication was restored to normal levels by interfering with MK2 activity (Figure 6.5 B). Corresponding to the rescue in DNA replication, we also expected that the cells would have a better proliferation rate and less yH2AX accumulation. To test this, we carried out immunostainings of $\mathrm{yH} 2 \mathrm{AX}$ and Celigo-based experiments. As a result, both the level of $\mathrm{yH} 2 \mathrm{AX}$ and cell proliferation were partially rescued by MK2 inhibition (Figure 6.5 C, D, and Figure $6.5 \mathrm{G}$ ). Despite the observed rescue of fork progression, accumulation of $\mathrm{yH} 2 \mathrm{AX}$, and cell proliferation, MK2 inhibition did not influence centrosome number (Figure 6.5 E, F).

Moreover, we performed parallel experiments upon depletion of centrosomal components and co-depletion of MK2 (Figure 6.5 H). Similarly, MK2 activity is highly required to interfere with the fork progression, accumulate $\mathrm{yH} 2 \mathrm{AX}$ as well as inhibit cell proliferation in this context (Figure $6.5 \mathrm{I}-\mathrm{K}$, Figure $6.5 \mathrm{~N}$ ) but its activity has no impact on the centrosome number (Figure 6.5 L, M).

On the contrary, overexpressing PLK4 led to enhanced fork progression even when cells were treated with gemcitabine through MK2 inhibition, a drug that induces replication stress (Figure 6.5 OQ). Thus, the observed activation of MK2 by centrosome disruption is an essential cause of the reduction in DNA replication fork progression, accumulation of $\mathrm{yH} 2 \mathrm{AX}$, and cell proliferation, but it has no impact on centrosome number. 
A

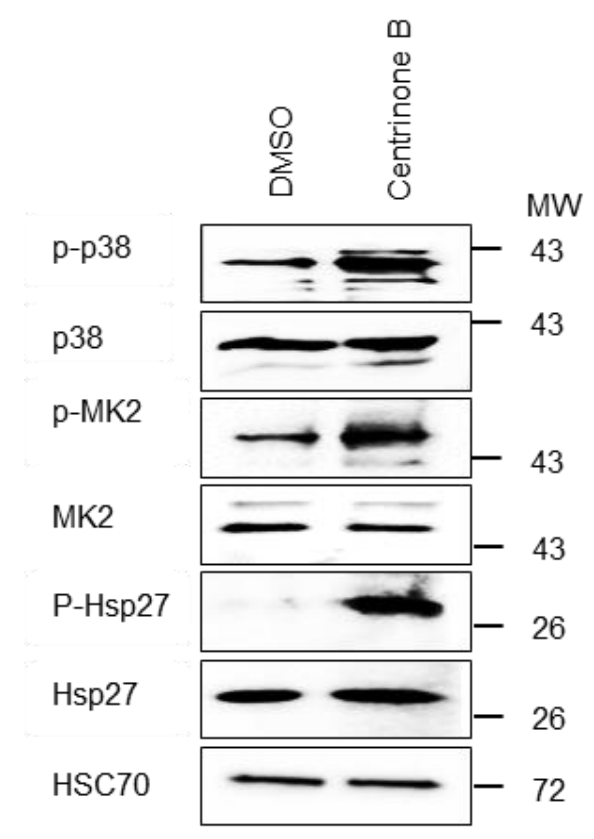

C

DAPI

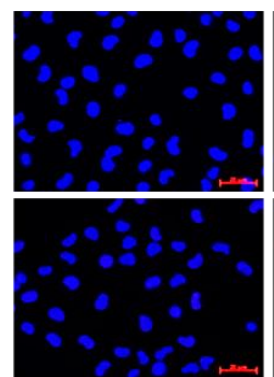

Centrinone B
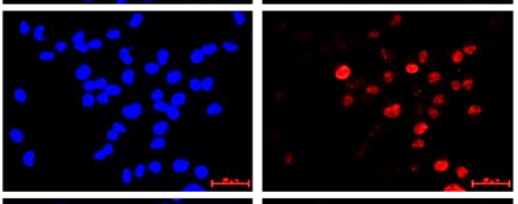

YH2AX

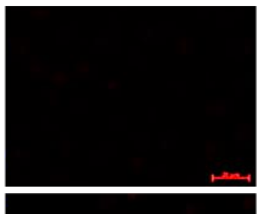

MK2i

Centrinone B MK2i

B

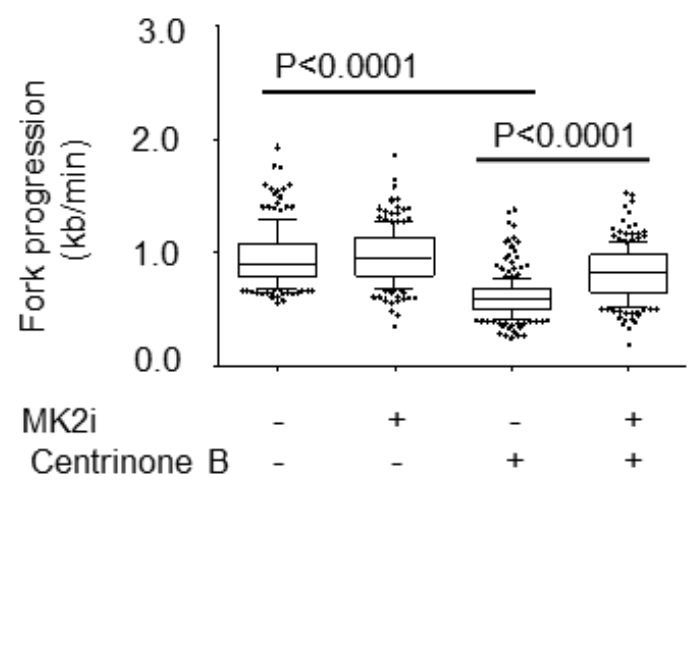

D

Merged
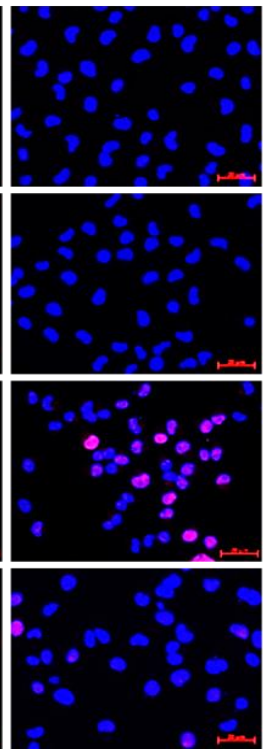

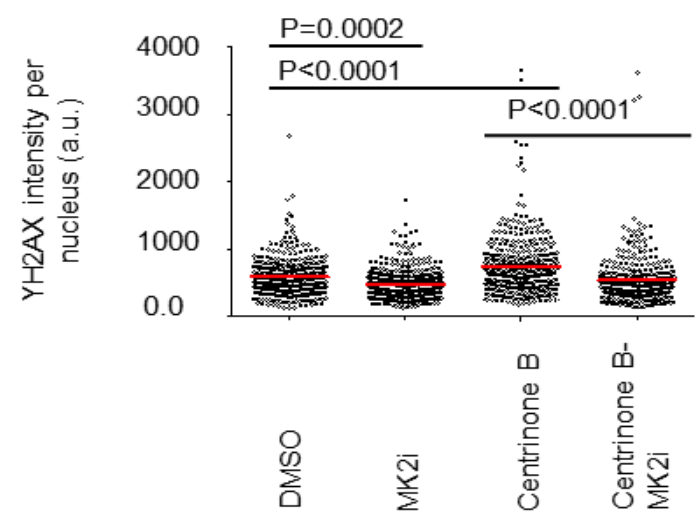


E

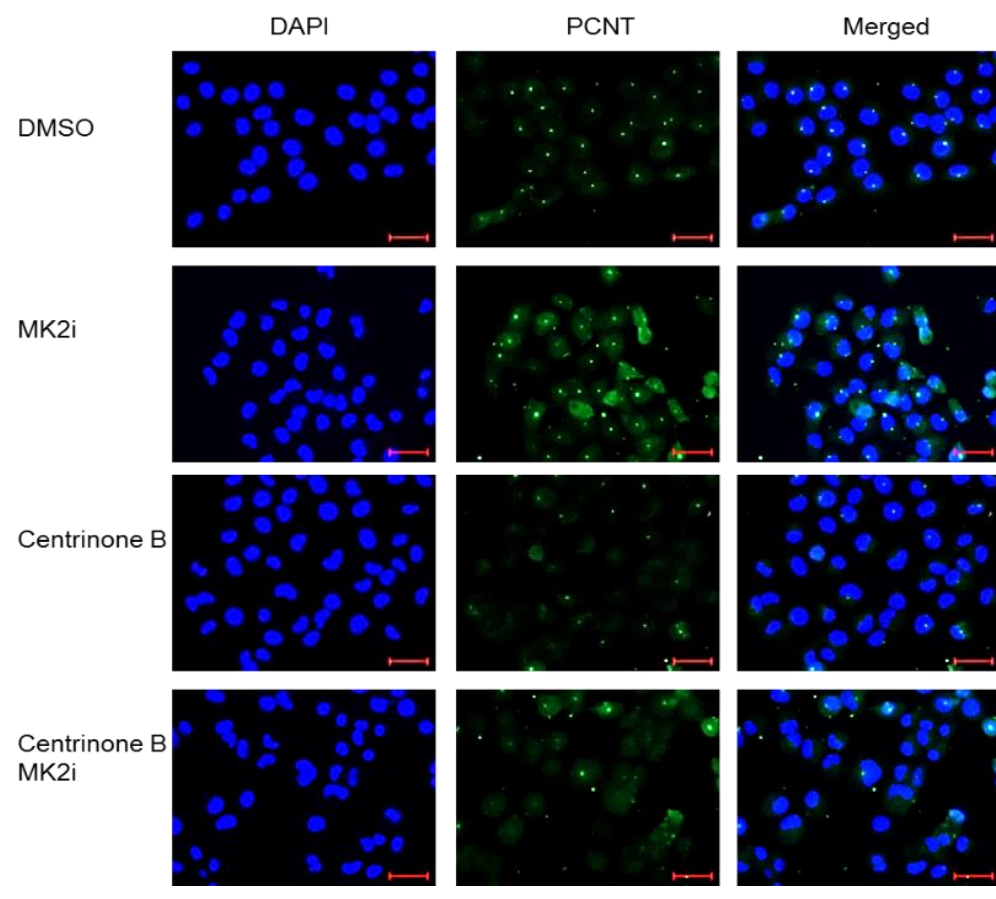

G

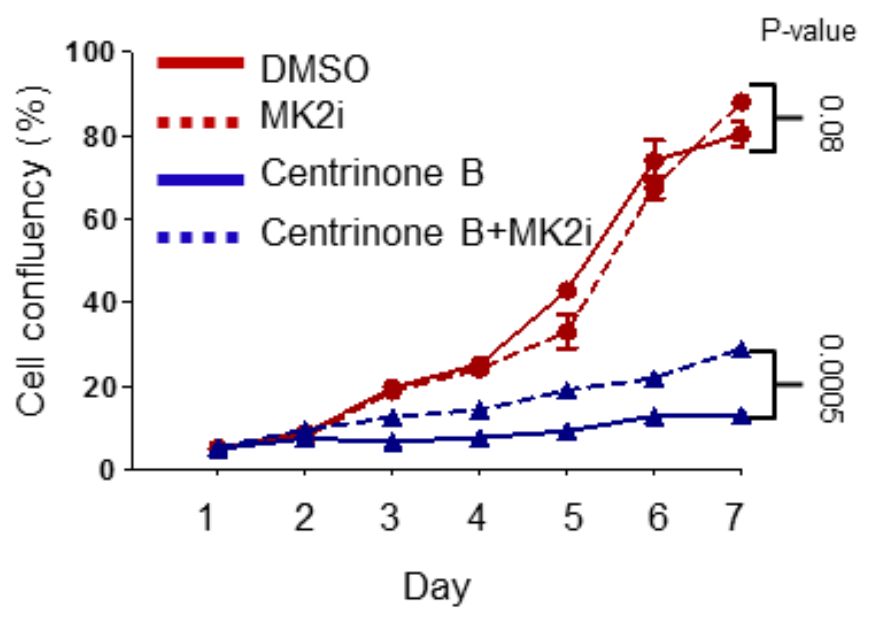

F

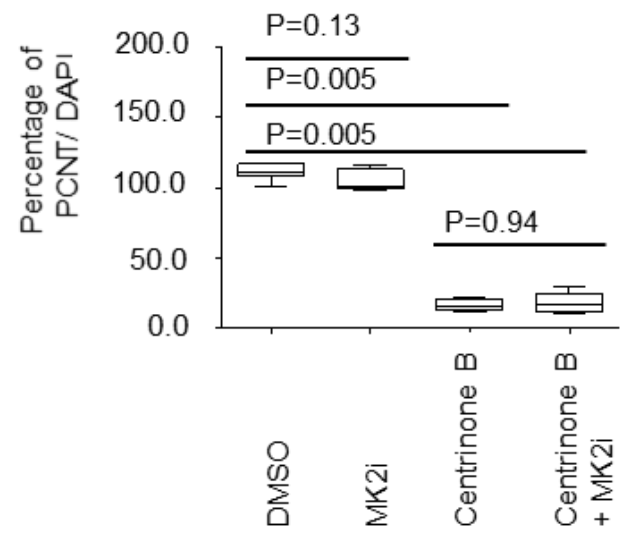

H

SiRNA

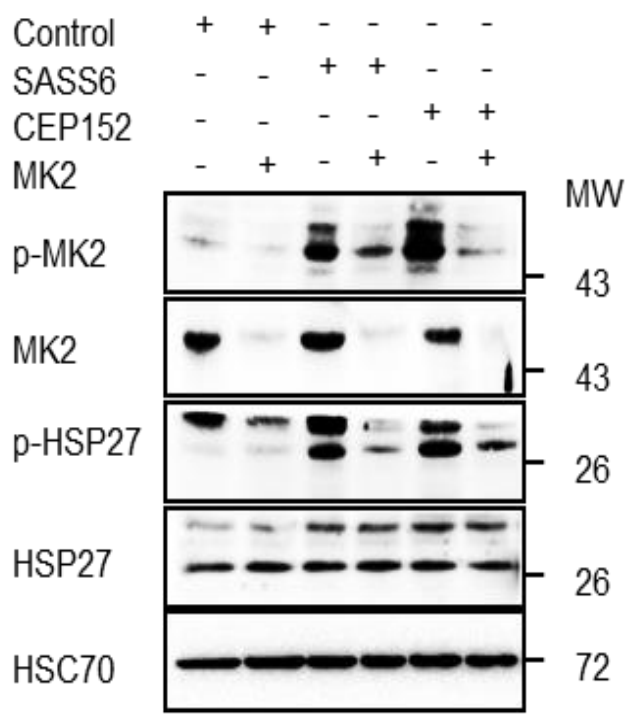


I

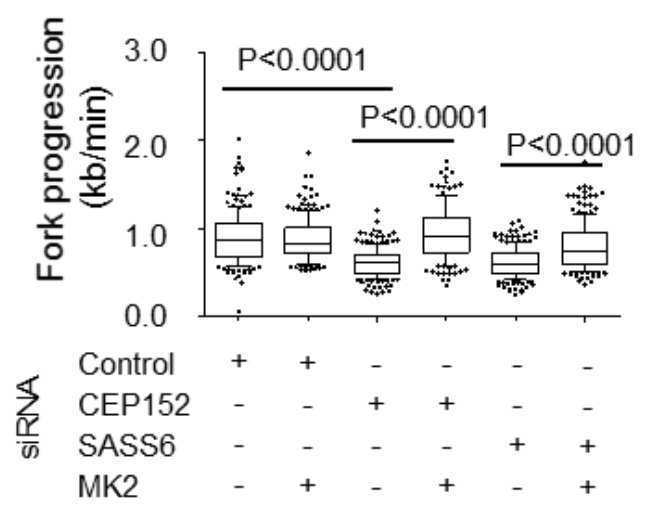

K
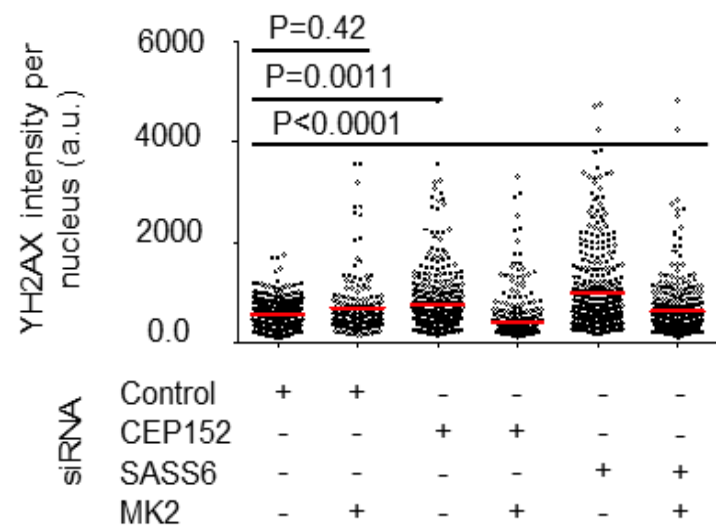

M

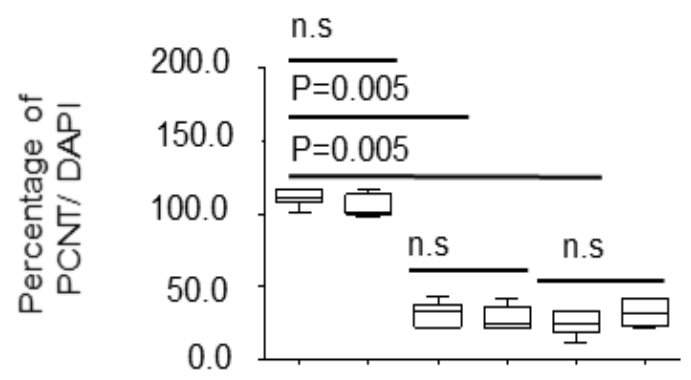

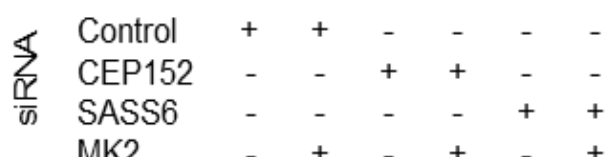

\section{J}

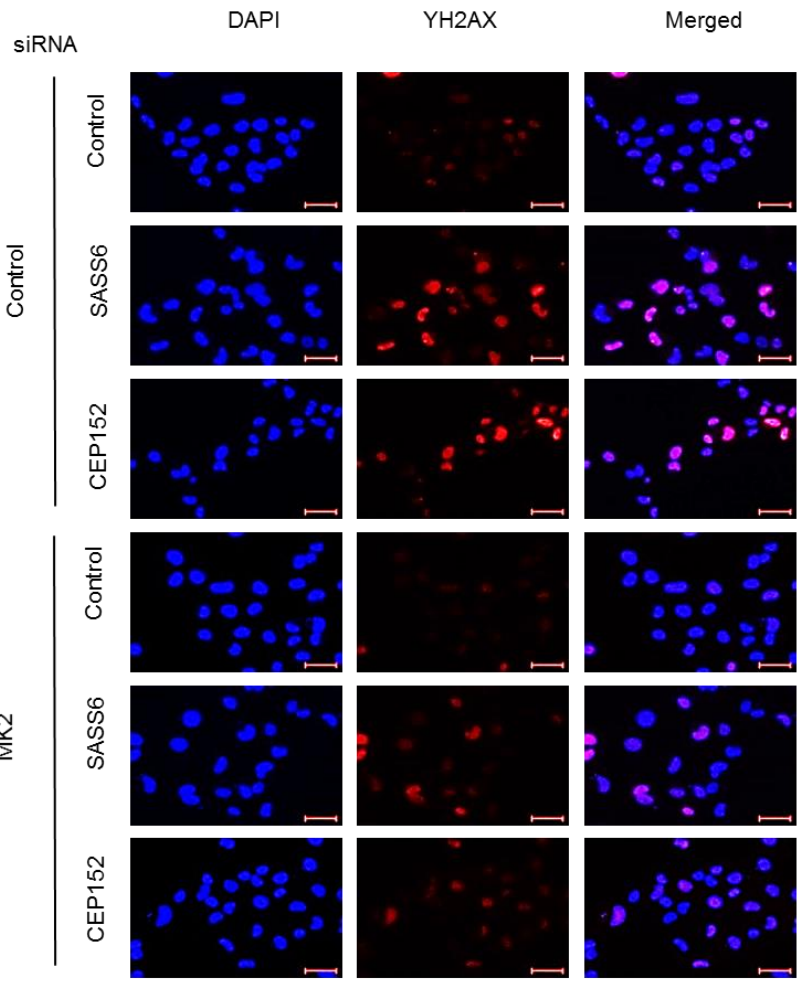

L

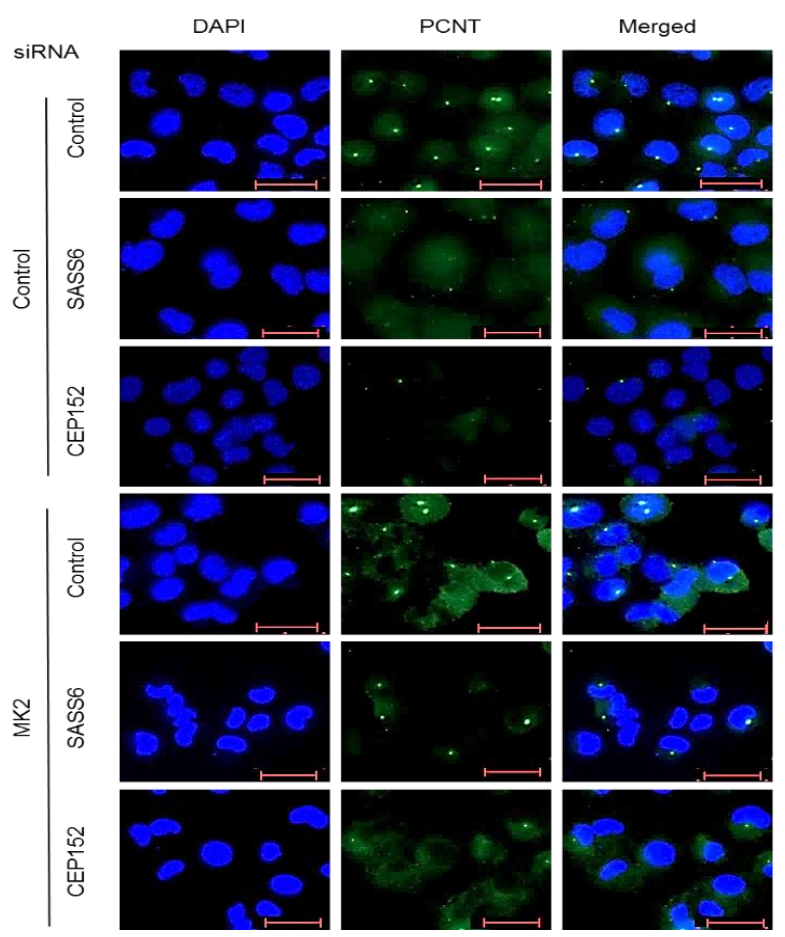



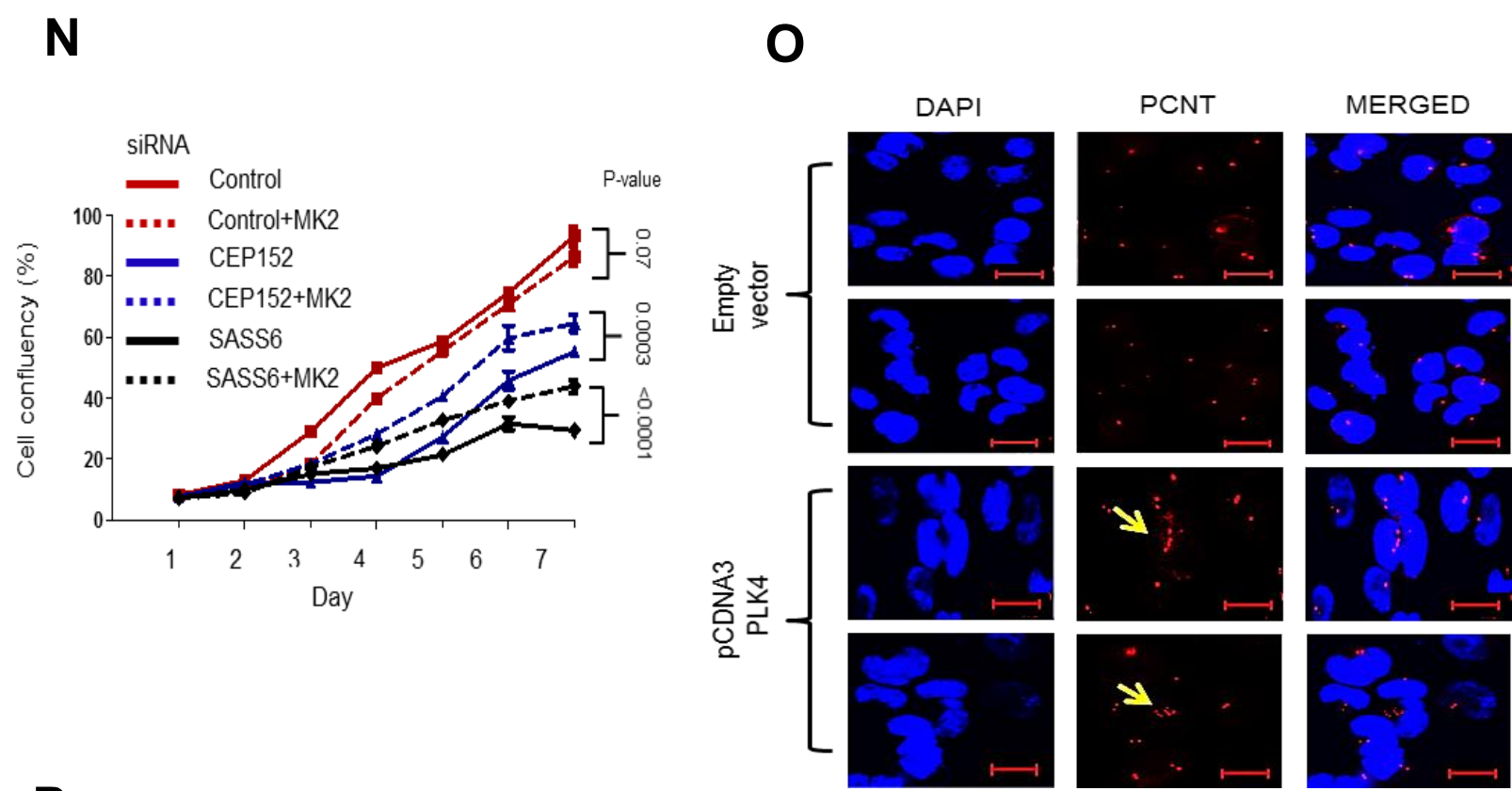

\section{P}

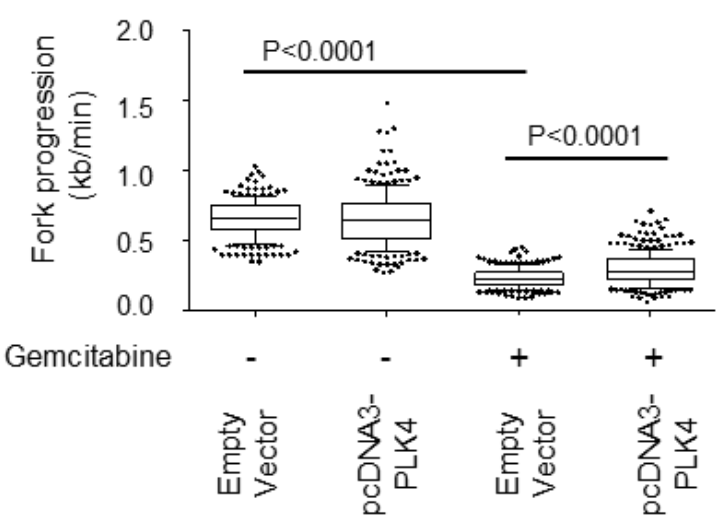

\section{Q}

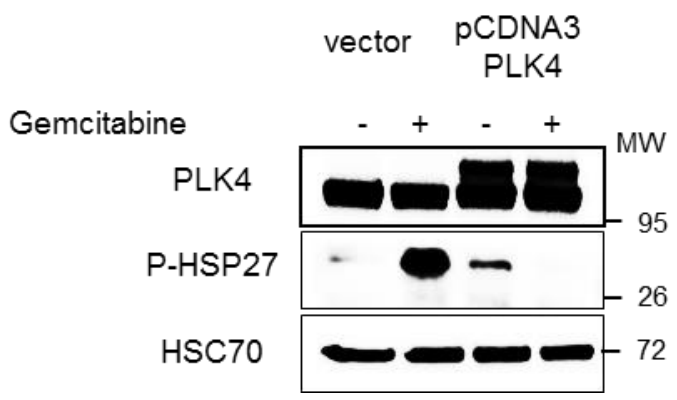


Figure 6.5: Centrosomal disintegration induces replication stress through p38 and MK2.

(A) Activation of the p38/MK2 pathway by PLK4 inhibition. Synchronized H1299 cells were treated with $500 \mathrm{nM}$ Centrinone B for 48 hours and analyzed by immunoblot. The phosphorylation of p38 and MK2, as well as the phosphorylation of the bona fide MK2 substrate HSP27, each indicate activation of the p38/MK2 signaling pathway.

(B) Synchronized H1299 cells were treated with $500 \mathrm{nM}$ Centrinone B for 48 hours and $10 \mu \mathrm{M}$ MK2 inhibitor MK2illl (termed MK2i from here on) for 24 hours, followed by fiber assays to quantitate DNA replication fork progression.

(C) Accumulation of $\mathrm{yH} 2 \mathrm{AX}$ signal in synchronized H1299 cells treated with PLK4 inhibitor with and without MK2i. Cells were treated with $500 \mathrm{nM}$ Centrinone $\mathrm{B}$ for 48 hours and $10 \mu \mathrm{M}$ MK2i for 24 hours followed by immunostaining for $\mathrm{yH} 2 \mathrm{AX}$, and the cell nuclei (DAPI) (scale bar $=20 \mu \mathrm{m})$.

(D) Quantification of the yH2AX signal in cell nuclei upon treatment with Centrinone B for 48 hours, followed by treatment with 10 $\mu \mathrm{M}$ MK2i inhibitor for 24 hours. The nuclear signal was quantified using the ImageJ software. The mean and distribution of three biological replicates (integrated) were calculated, and the Mann-Whitney t-test assessed the significance.

(E) MK2 activity has no impact on centrosome numbers. Synchronized $\mathrm{H} 1299$ cells were treated as described in B, then subjected to centrosome immunostaining for PCNT as a marker, and the cell nuclei (DAPI) (scale bar $=20 \mu \mathrm{m}$ ).

(F) One hundred fifty cells were counted per each condition from $\mathrm{E}$, and an average of three biological replicates are presented.

(G) Partial rescue on cell proliferation upon PLK4 inhibition by MK2 inhibition. 5*103 H1299 cells were seeded in each well of a 24well plate. Cells were treated with DMSO or $300 \mathrm{nM}$ Centrinone B, with or without $10 \mu \mathrm{M}$ MK2i. Confluence was measured every 24 hours for seven days using the CeligoTM Cytometer (Nexcelom, software version 2.0). The experiment was carried out in three biological replicates and six technical replicates for each time point. Note that most error bars are too narrow to be displayed.

(H) Rescue of the observed activation of MK2 upon centrosome depletion, using MK2 knockdown. H1299 cells were reverse transfected with $10 \mathrm{nM}$ pooled siRNAs against CEP152 and SASS6, in combination with siRNAs to MK2, for 72 hours, followed by immunoblot analyses.

(I) MK2 dependence of replication stress upon centrosome depletion. Synchronized H1299 cells were transfected as described in $(\mathrm{H})$. Cells were subjected to fiber assays, as described in (B).

(J) Accumulation of $\mathrm{yH} 2 \mathrm{AX}$ signal in synchronized $\mathrm{H} 1299$ cells transfected with $10 \mathrm{nM}$ pooled siRNAs against CEP152 and SASS6, in combination with siRNAs to MK2, for 72 hours followed by immunostaining for yH2AX and the cell nuclei (DAPI) (scale bar = $20 \mu \mathrm{m})$.

(K) Quantification of the $\mathrm{yH} 2 \mathrm{AX}$ signal in cell nuclei upon depletion of centrosomal components. $\mathrm{H} 1299$ cells were reverse transfected with $10 \mathrm{nM}$ pooled siRNAs, in combination with siRNAs to MK2, for 72 hours, followed by immunostaining. The nuclear signal was quantified using the ImageJ software. The mean and distribution of three biological replicates (integrated) were calculated, and the Mann-Whitney test assessed the significance.

(L) MK2 activity has no impact on centrosomes numbers. Synchronized $\mathrm{H} 1299$ cells were treated as described in $(\mathrm{H})$, then subjected to centrosome immunostaining with PCNT as a marker, and the cell nuclei (DAPI) were detected (scale bar $=20 \mu \mathrm{m}$ ).

(M) One hundred fifty cells were counted per each condition from (E), and an average of three biological replicates are presented.

(N) Rescue of cell proliferation upon centrosome depletion using MK2 knockdown. H1299 cells were reverse transfected with $10 \mathrm{nM}$ of siRNA as in $(\mathrm{H})$, followed by an assessment of cell proliferation as in $(\mathrm{G})$ with three biological replicates and six technical replicates for each time point.

(0) Increased centrosome formation upon PLK4 overexpression. Synchronized H1299 cells were subjected to plasmid transfection (pcDNA3, pcDNA3-PLK4) for 48 hours. Centrosomes were detected by immunostaining of PCNT, and the DAPI signal was used to identify the nuclei. Scale bar represents $20 \mu \mathrm{m}$.

(P) PLK4 overexpression partially rescues DNA replication in gemcitabine-treated cells. Synchronized H1299 cells were subjected to plasmid transfection (pcDNA3, pcDNA3-PLK4) for 48 hours, followed by treatment with $300 \mathrm{nM}$ gemcitabine for 2 hours before harvesting. DNA replication fork progression was determined using fiber assays.

(Q) PLK4 overexpression diminishes MK2 activation in the presence of gemcitabine. Immunoblot analysis was performed to confirm PLK4 overexpression (note that the apparent molecular weight is increased due to the Flag tag). MK2 activity, as revealed by HSP27 phosphorylation, is enhanced by gemcitabine, as found earlier, but not when PLK4 was overexpressed. 


\subsection{Upon centrosome disruption, the kinase MLK3 activates p38 and MK2}

Taking into account the crucial function of MK2 in replication stress, we sought to determine the upstream signaling pathway that leads to its activation in response to centrosome disruption. An upstream kinase of p38 that was previously found to be associated with the centrosome is MLK3 (130), a member of the serine/threonine kinase family, and contains an SH3 domain and a leucine zipper-basic motif. MLK3 known with its ability to activate MAPK8/JNK kinase and functions as a positive regulator of the JNK signaling pathway. MLK3 is also essential for the activation of MAPK14 (p38), MAPK3 (ERK), and MAPK8 (JNK1) through phosphorylation and activation of MAP2K4/MKK4 and MAP2K7/MKK7. Also, it is known to have an influence on microtubule organization during the cell cycle. MLK3 can undergo dimerization during activation and interacts with MAP2K4/MKK4, MAP2K7/MKK7. It was also found in a complex with SH3RF1, RAC1, MAP2K7/MKK7, MAPK8IP1/JIP1, and MAPK8/JNK1 (101,115,118). Therefore, we hypothesized that the activation of centrosomal MLK3 is a reasonable way to activate p38/MK2 (Figure 6.6 A).

Accordingly, we found that the association of MLK3 with centrosomal structures got lost in cells treated with the PLK4 inhibitor Centrinone B (Figure 6.6 B, C). Strikingly, MLK3 inhibition prevented the accumulation of phosphorylated p38 and MK2, which otherwise occurred upon PLK4 inhibition (Figure 6.6 D), in agreement with an earlier report suggesting this possibility (130). Moreover, MLK3 inhibition ultimately rescued DNA replication fork progression and accumulation of $\mathrm{yH} 2 \mathrm{AX}$ in the presence of the PLK4 inhibitor (Figure 6.6 E-G). Not only this, but it also seems that the activity of MLK3 has an impact on centrosomes number (Figure $6.6 \mathrm{H}, \mathrm{I}$ ). In the same way, MLK3 depletion largely restored DNA replication and decreased the level of $\mathrm{yH} 2 \mathrm{AX}$ when centrosomal components were knocked down (Figure 6.6 K-M).

Moreover, it prevented p38/MK2 activation (Figure 6.6 J). In contrast to MK2 activation in the context of centrosomes number, MLK3 seems to play a crucial role in determining the centrosomes number under stress conditions (Figure $6.6 \mathrm{~N}, \mathrm{O}$ ). Thus, taking into account all of the results mentioned above, we conclude that similarly to MK2, MLK3 is required for a signal triggered by centrosome disruption to interfere with DNA replication and to maintain centrosomes number. MLK3 operates by activating $\mathrm{p} 38$ and MK2, which then cause replication stress. 


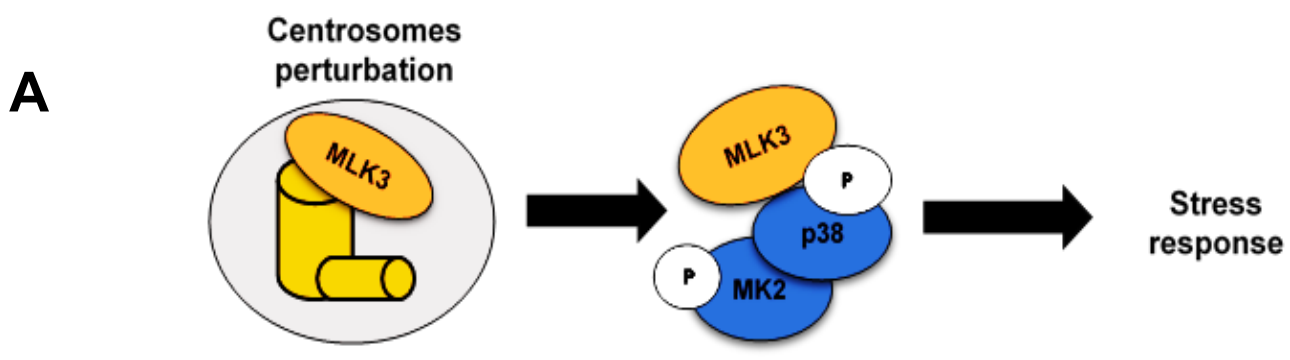

B

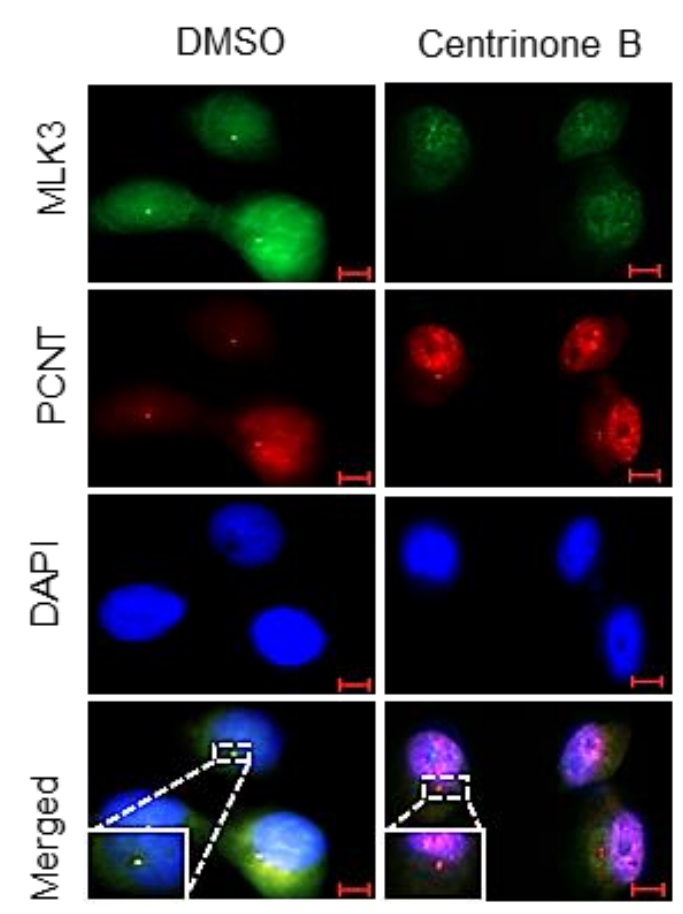

C
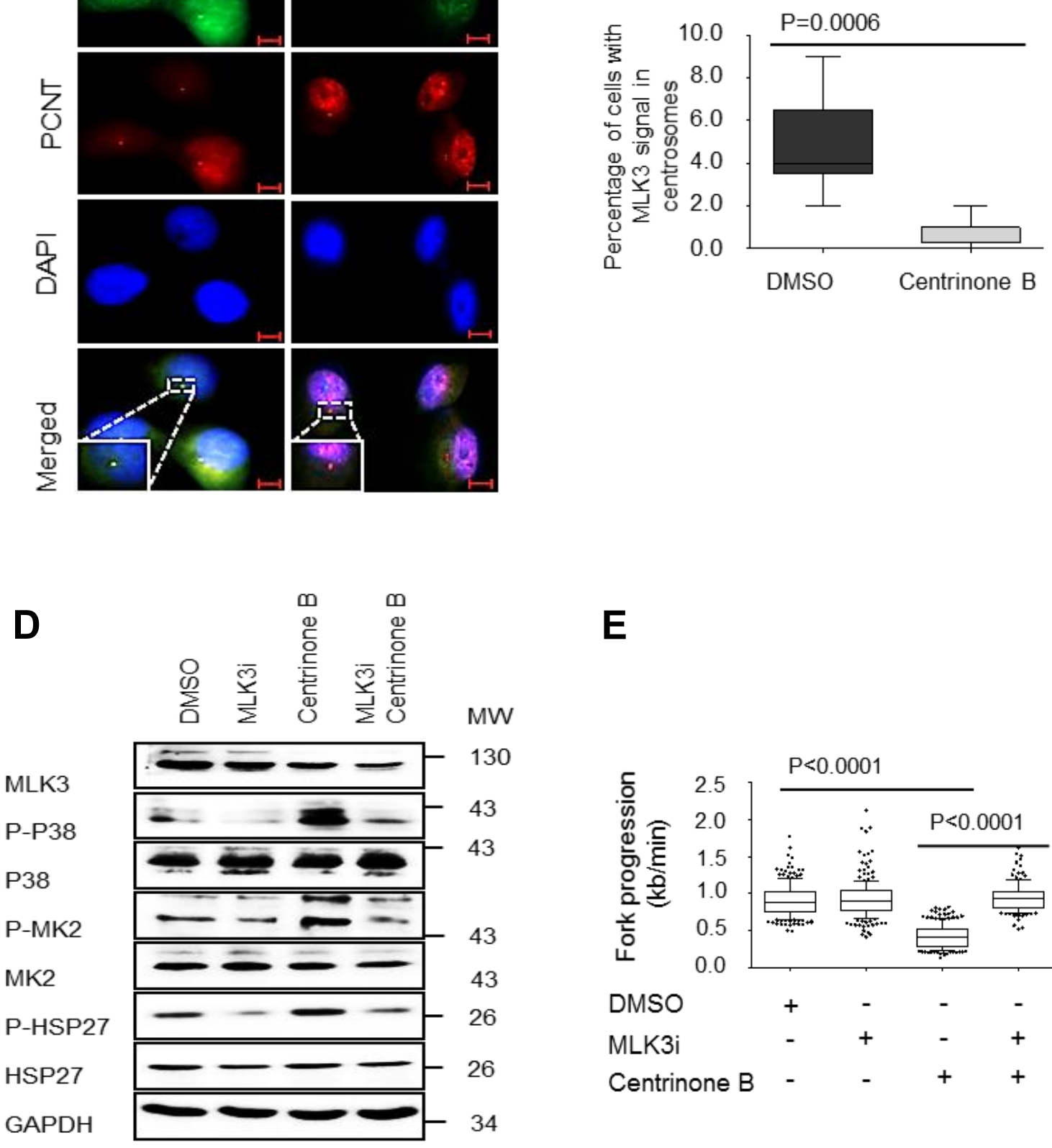

E

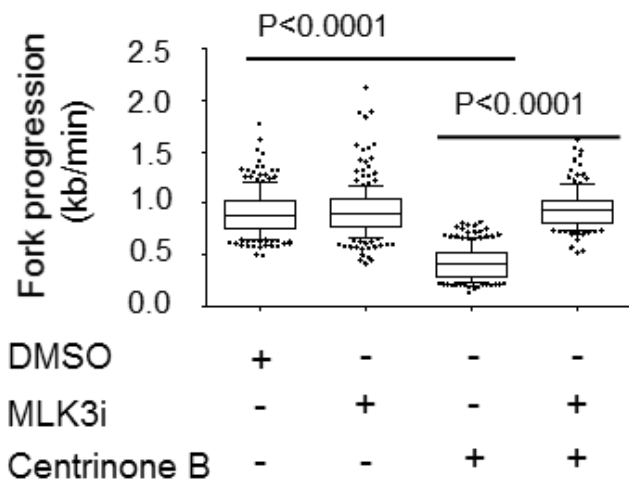


F

G

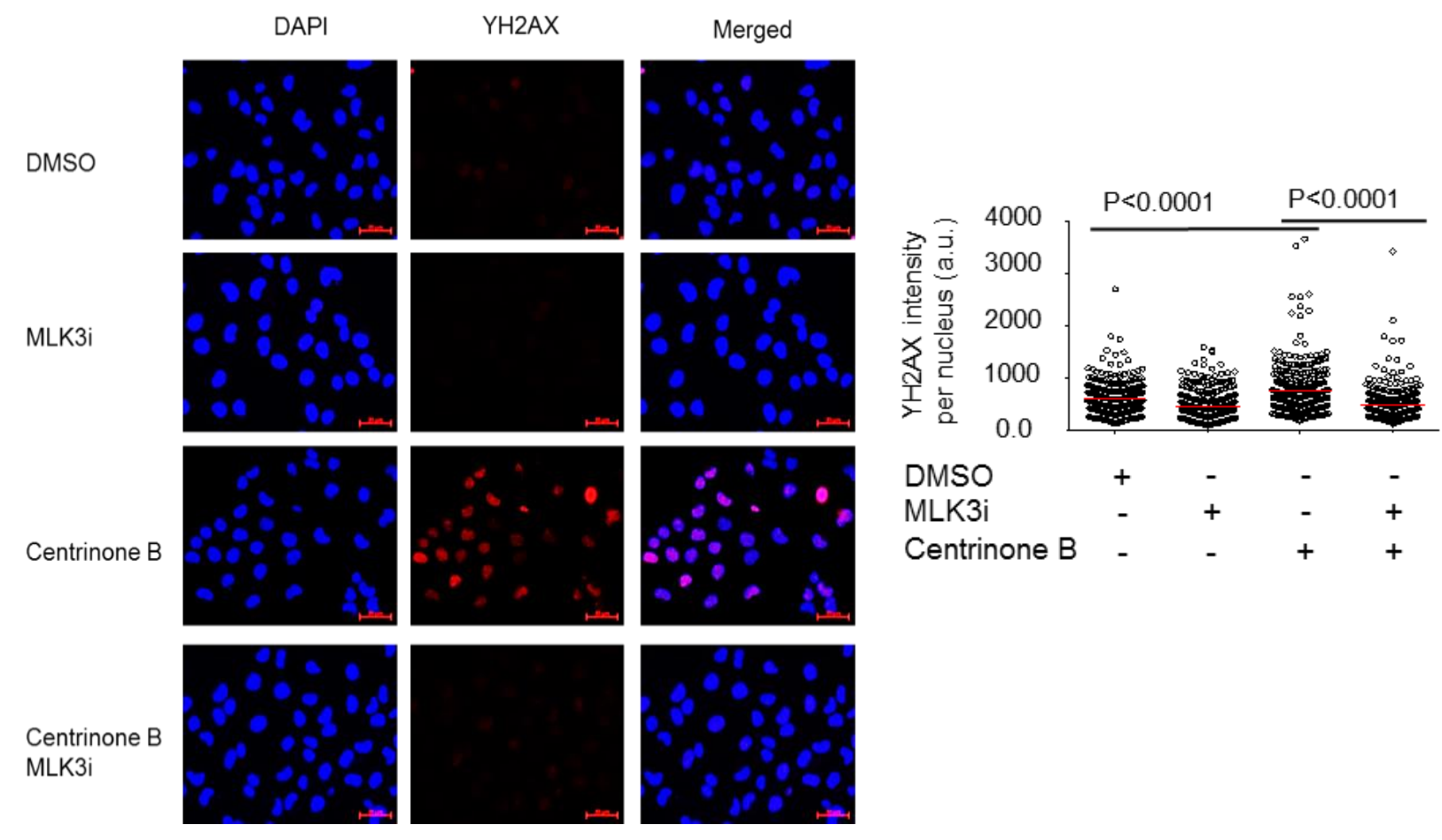

H

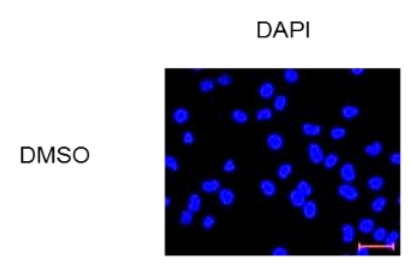

PCNT
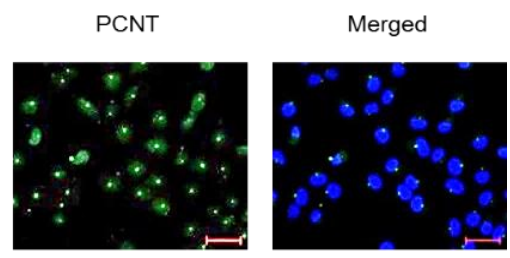

I
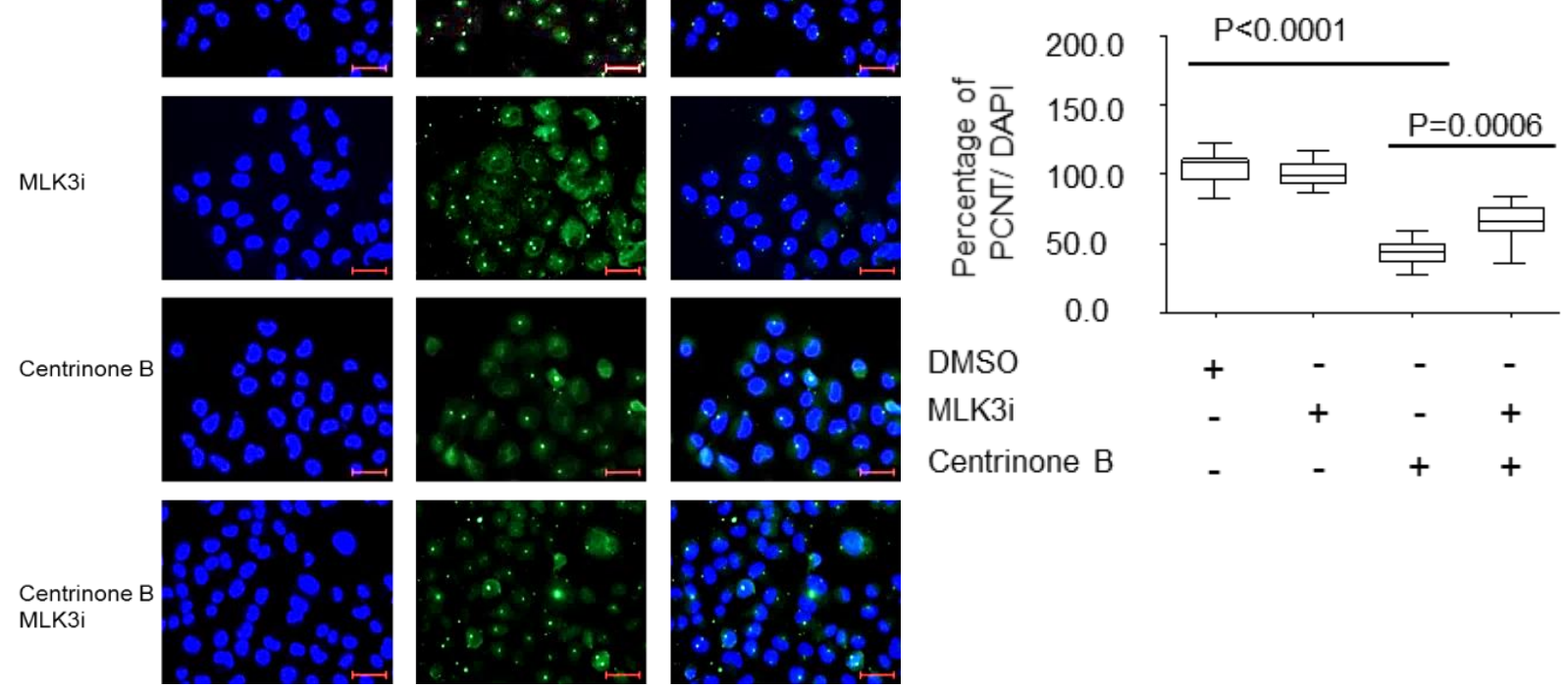

DMSO

MLK3i

Centrinone B

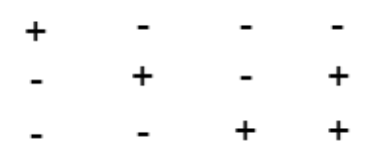


J

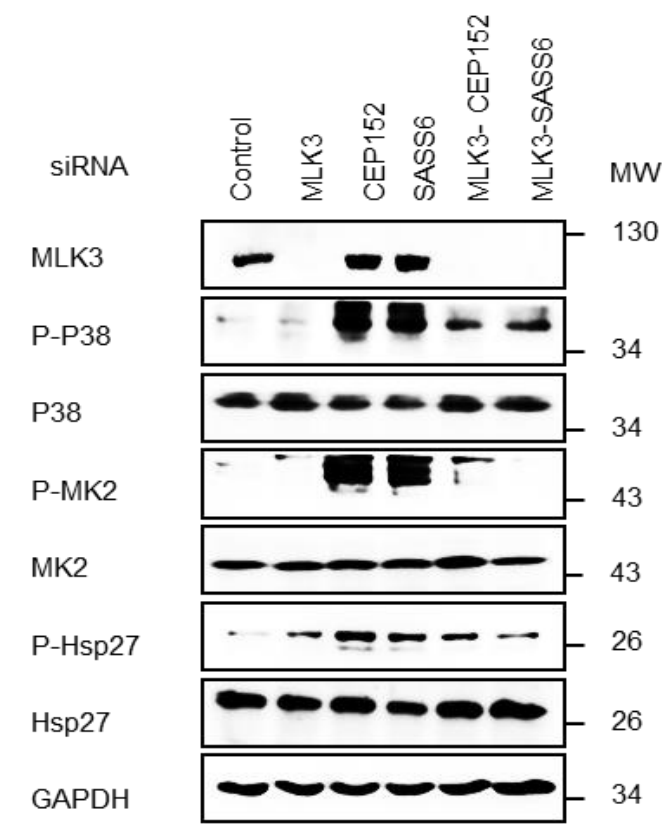

L

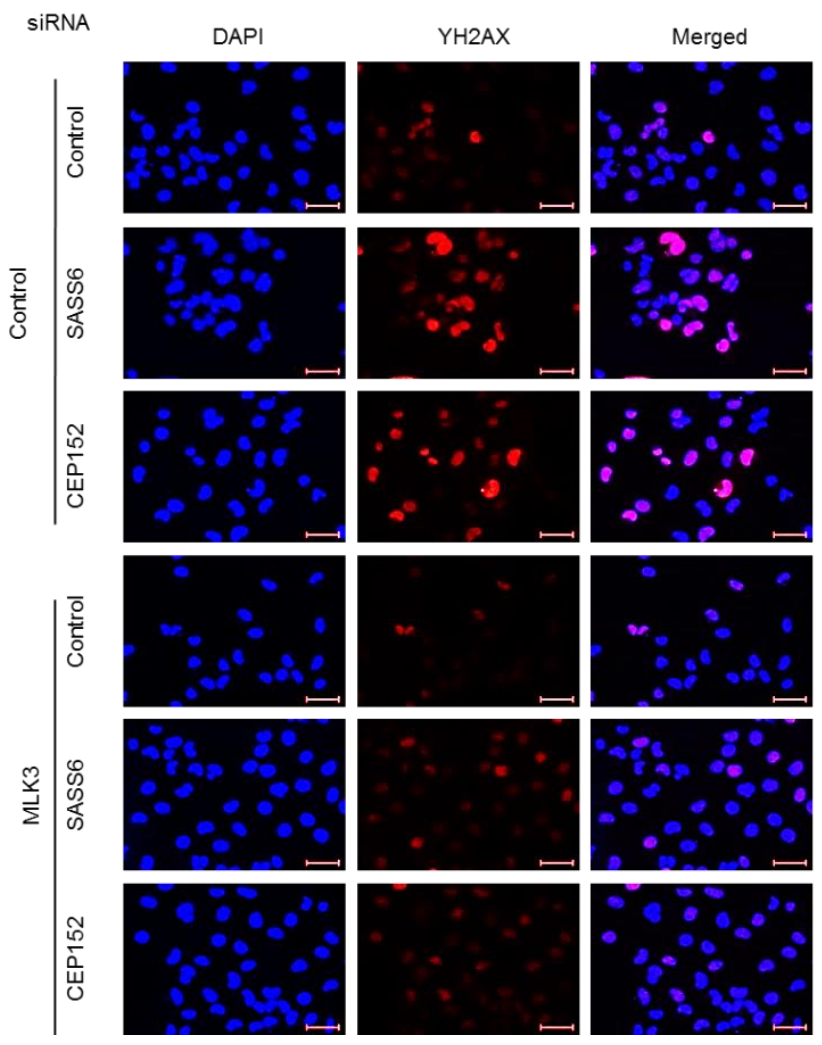

K

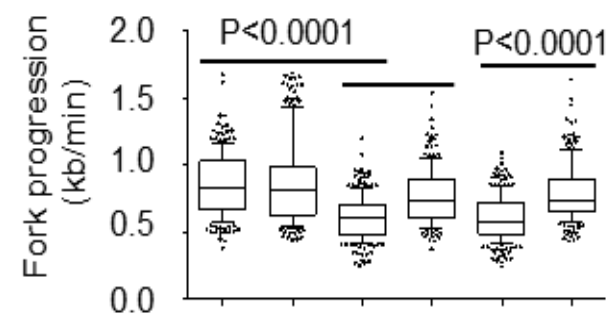

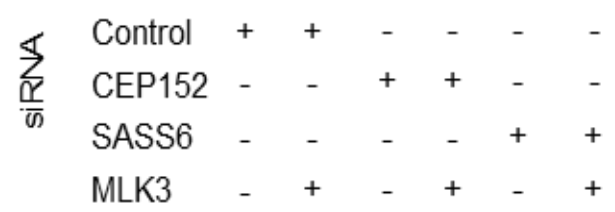

M
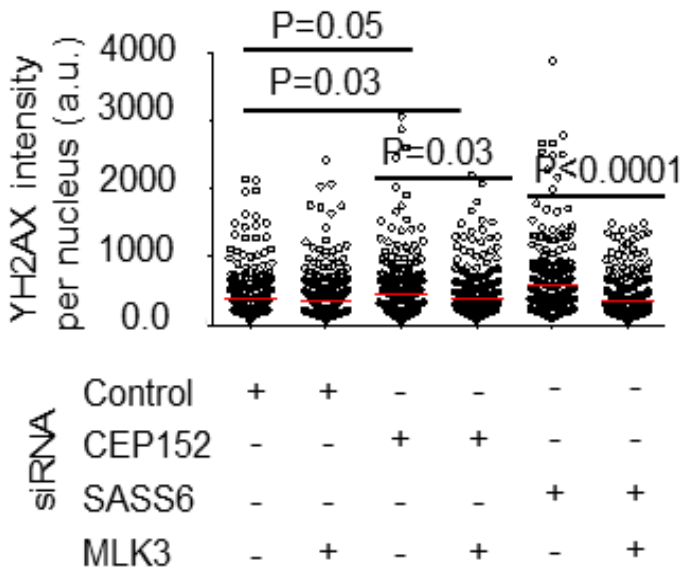
$\mathbf{N}$

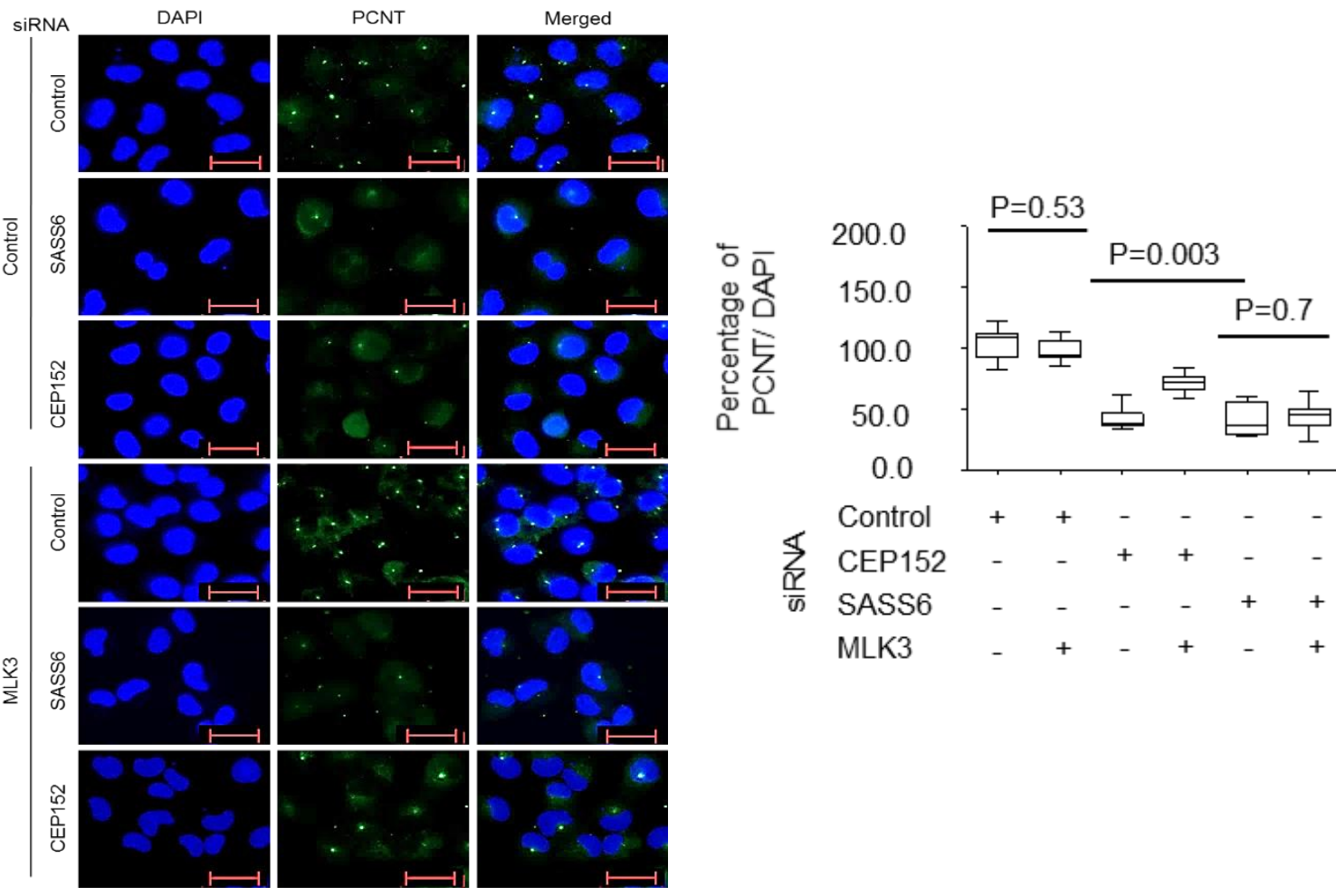

Figure 6.6: When centrosomes are disintegrated, MLK3 activates p38/MK2 to reduce fork progression.

(A) Schematic diagram presenting a potential interaction between MLK3/P38/MK2.

(B) MLK3 associates with centrosomes in a PLK4-dependent manner. Synchronized H1299 cells were treated with either DMSO or $500 \mathrm{nM}$ Centrinone B for 48 hours. Centrosomes were stained to detect PCNT and MLK3. DAPI was used to stain the nuclei (scale bar $=20 \mu \mathrm{m}$ ).

(C) Quantification of MLK3 association to the centrosomal PCNT signal. Three hundred nuclei were quantified per condition. The results represent the quantification of two technical replicates and an average of three biological replicates. Some cells treated with Centrinone B did not lose the centrosome signal (PCNT), and these were the cells included in the analyses regarding the co-localization of centrosomes with MLK3 in our quantification. $(n=3)$, ${ }^{* \star *} \mathrm{P}<0.0001$.

(D) Dependence of p38/MK2 activation on MLK3. Synchronized H1299 cells were treated with $500 \mathrm{nM}$ Centrinone B for 48 hours. During the last 24 hours, the MLK3 inhibitor URMC-099 was added at $200 \mathrm{nM}$ for 24 hours, followed by immunoblot analysis.

(E) Rescue of DNA replication by MLK3 inhibition in cells treated with PLK4 inhibitor. Synchronized cells were treated as described in (B) followed by DNA fiber assays, which were performed to assess replication fork progression.

(F) Accumulation of $\mathrm{yH} 2 \mathrm{AX}$ signal in synchronized H1299 cells treated with PLK4 inhibitor with and without MLK3i. Cells were treated with $500 \mathrm{nM}$ Centrinone B for 48 hours and $200 \mathrm{nM}$ MLK3i for 24 hours, followed by immunostaining for $\mathrm{yH} 2 \mathrm{AX}$, and the cell nuclei (DAPI) were detected (scale bar $=20 \mu \mathrm{m}$ ).

(G) Quantification of the yH2AX signal in cell nuclei upon treatment with Centrinone B for 48 hours, followed by treatment with 200 nM MLK3 inhibitor for 24 hours. The nuclear signal was quantified using the ImageJ software. The mean and distribution of three biological replicates (integrated) were calculated, and the Mann-Whitney test assessed the significance.

(H) MLK3 activity has a partial impact on centrosomes number. Synchronized H1299 were treated as in (E). Cells were subjected to immunostaining with PCNT as a marker, and the cell nuclei (DAPI) was detected (scale bar $=20 \mu \mathrm{m}$ ).

(I) Quantification of the centrosome signals per cell to DAPI-stained nuclei. One hundred fifty cells were quantified per condition and presented as percentage (the number of detectable centrosomes divided by the number of nuclei, multiplied by $100 \%$ ) using GraphPad Prism. $(n=3),{ }^{* * * *} P<0.0001$. 
(J) MLK3 knockdown diminishes p38/MK2 activation upon centrosome depletion. Synchronized H1299 cells were reverse transfected with $10 \mathrm{nM}$ siRNAs against the targets CEP152, SASS6, and MLK3 for 72 hours. Phospho-specific antibodies were used to detect pathway activation.

(K) MLK3 depletion rescued DNA replication when pooled siRNAs removed centrosomal components. Synchronized cells were treated as in $(\mathrm{J})$ and then subjected to DNA fiber assays.

(L) Accumulation of $\mathrm{yH} 2 \mathrm{AX}$ signal in synchronized $\mathrm{H} 1299$ cells treated as in (J), followed by immunostaining against $y \mathrm{H} 2 \mathrm{AX}$ and the cell nuclei (DAPI) (scale bar $=20 \mu \mathrm{m})$.

(M) Quantification of the yH2AX signal in cell nuclei upon centrosomal components depletion for 72 hours with and without MLK3 knockdown. The nuclear signal was quantified using the ImageJ software. The mean and distribution of three biological replicates (integrated) were calculated, and the significance was assessed by the Mann-Whitney test.

(N) MLK3 activity has a partial impact on centrosomes numbers after knocking down CEP152 but not after knocking SASS6. Synchronized $\mathrm{H} 1299$ cells were treated as in (J), then subjected to centrosome immunostaining with PCNT and DAPI (scale bar $=20 \mu \mathrm{m})$.

(O) Quantification of the centrosome signals per cell to DAPI-stained nuclei. Synchronized H1299 cells treated as in (N). One hundred fifty cells were quantified per condition and presented as percentage (the number of detectable centrosomes divided by the number of nuclei, multiplied by $100 \%$ ) using GraphPad Prism. ( $n=3)$, ${ }^{* \star *} P<0.0001$.

\subsection{Centrosome disintegration induces the formation of RNA: DNA hybrids that are required for replication stress}

Replication stress is often driven by unscheduled transcription and the formation of R-loops, i. e. RNA hybridizing to DNA (often in association with transcription) and displacing the opposite DNA strand (60) (Figure 6.7 A). Accordingly, upon PLK4 inhibition, we detected the formation of RNA: DNA hybrids using immunostaining with the monoclonal antibody $\$ 9.6$ directed against these structures (Figure 6.6 B, C). Upon staining fixed cells in situ, the immunofluorescence signal derived from antibody binding was prominent in discrete nuclear structures, compatible with the concept that R-loops mainly occur at specific sites of highly active transcription (131-134). In contrast, the overexpression of RNaseH1, an RNase that cleaves the RNA component of RNA DNA hybrids, sharply reduced the nuclear immunostaining signal, confirming the specificity of the antibody (Figure 6.7 B, C). Similarly, in the dot blot analyses, the accumulation of R-loops was also observed upon PLK4 inhibition as well as centrosomes depletion (Figure 6.7 D-G), and RNAseH1 treatment sharply reduced the signal (Figure 6.7 D, E), confirming that RNA: DNA hybrids are the source of the antibody signal. Interestingly, the inhibition of p38/MK2 signaling also diminished the formation of R-loops upon centrosomal impairment, consistent with the rescue of DNA replication by the same inhibitors (Figure 6.7 H-K).

To clarify the causal link between R-loop formation and replication stress, we performed DNA fiber assays. Upon PLK4 inhibition, the progression of the DNA replication forks was largely rescued by $\mathrm{RNaseH1}$ overexpression (Figure $6.7 \mathrm{~L}, \mathrm{M}$ ). We conclude that interfering with centrosomal 
integrity not only induces R-loop formation but that this is a significant cause of the observed DNA replication stress.

A

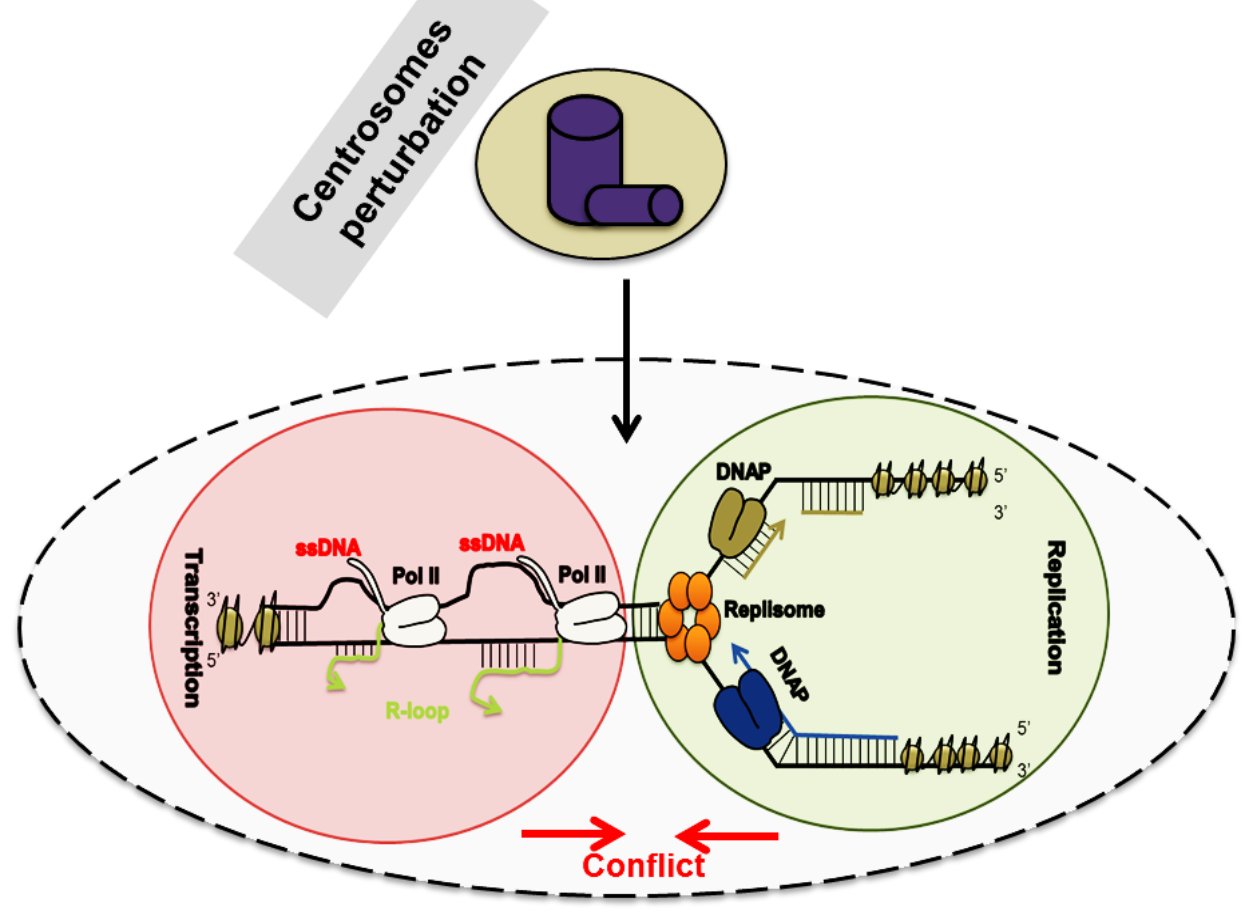

B

C

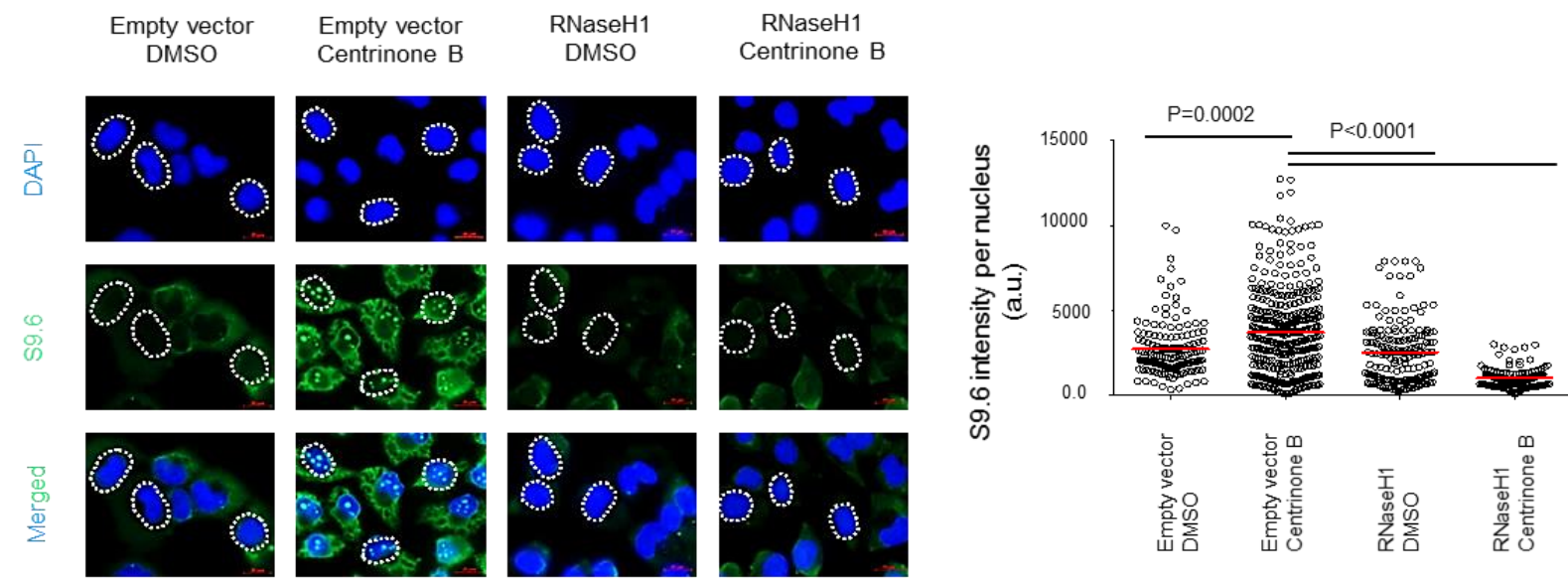


D

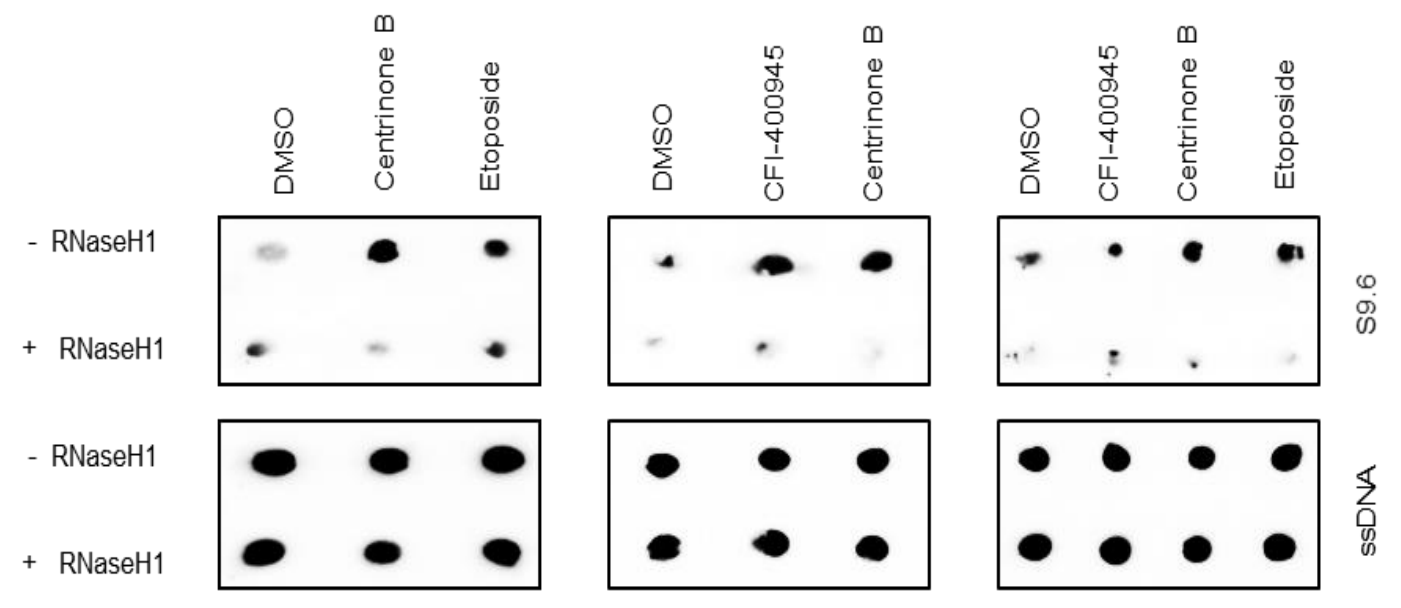

E
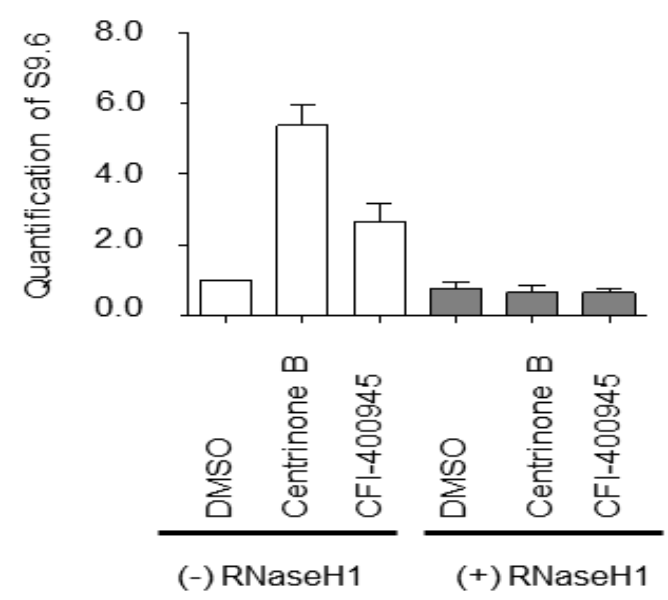

G

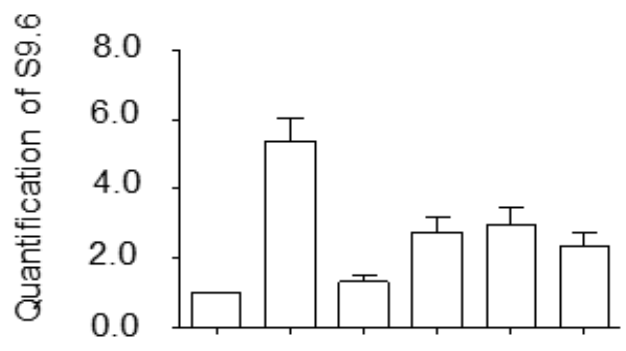

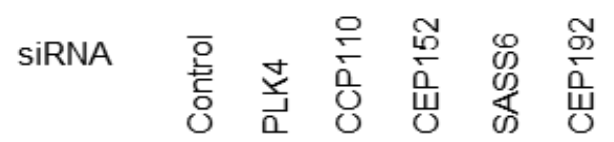

F
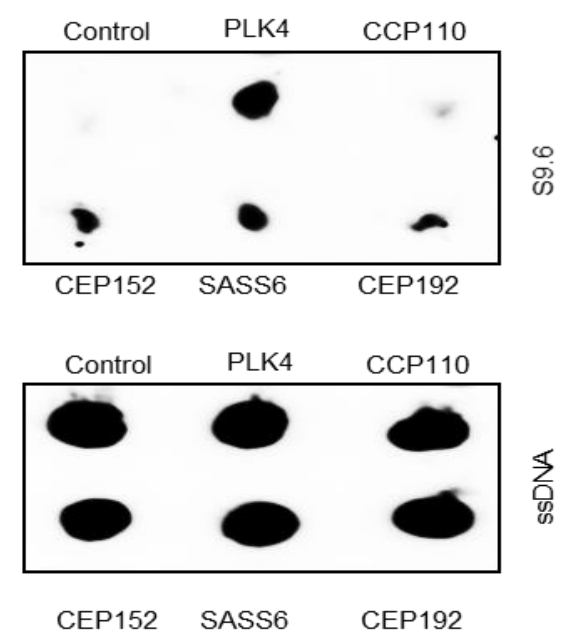

H

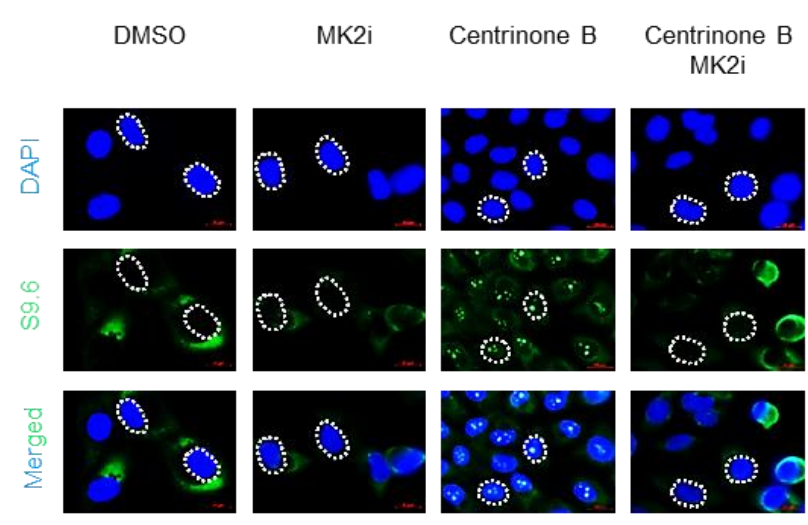




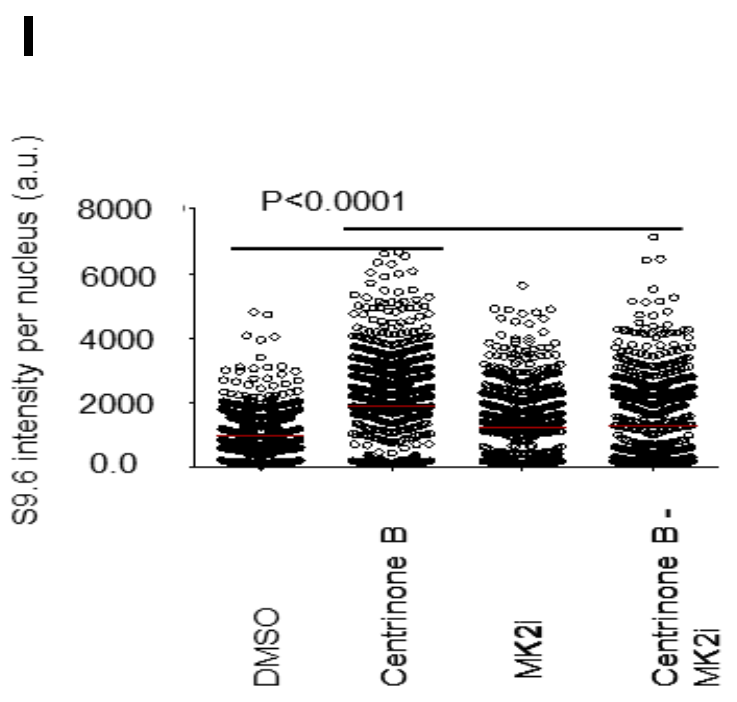

K

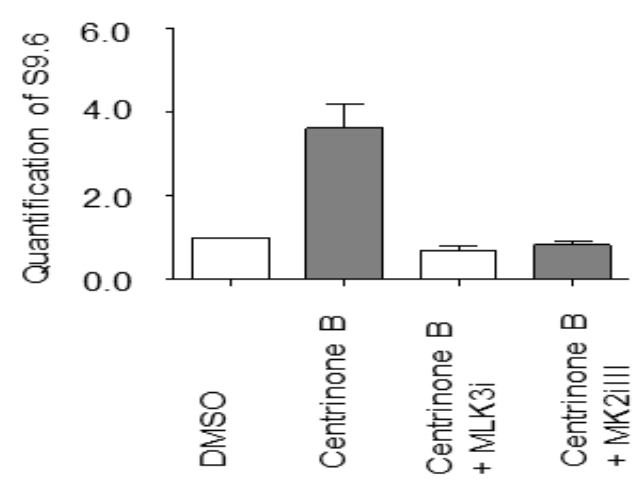

M

Vector RNaseH1

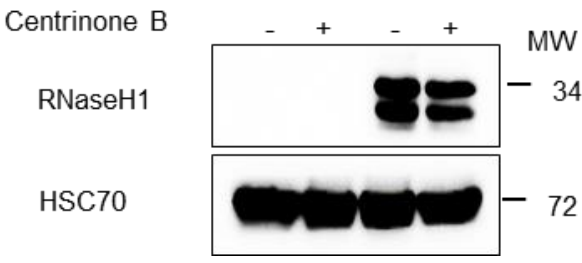

J
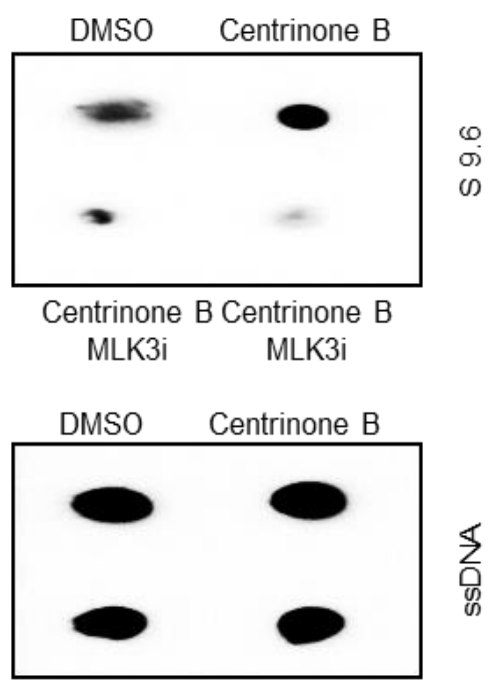

Centrinone B Centrinone B MLK3i MLK3i

$\mathbf{L}$

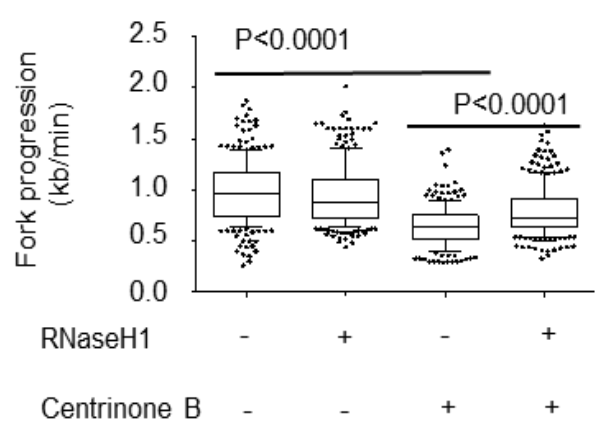

Figure 6.7: Replication stress upon centrosome disintegration requires RNA:DNA hybrids.

(A) The schematic diagram explains some factors leading to unscheduled replication-transcription conflicts.

(B) Immunostaining of the R-loop signal in cell nuclei upon treatment with Centrinone B and/or synchronized H1299 cells transfected with a control plasmid (PFRT-ToDestFlag-HA) or an RNaseH1 expression plasmid (PFRT-ToDestFLAG-HA-RNAseH1) for 48 
hours, along with $500 \mathrm{nM}$ Centrinone B treatment. The nuclei (DAPI) and RNA: DNA hybrids (antibody S9.6) were detected (scale bar $=20 \mu \mathrm{m})$.

(C) Quantification of the R-loop signal in cell nuclei upon treatment with Centrinone B and/or transfection with an expression plasmid for RNaseH1. The nuclear signal was quantified using the ImageJ software. The mean and distribution of three biological replicates (integrated) were calculated, and the significance was assessed by the Mann-Whitney test.

(D) Dot-blot analysis of RNA: DNA hybrids in synchronized H1299 cells. Cells were treated either with DMSO, 500 nM Centrinone $\mathrm{B}$, or $10 \mathrm{nM} \mathrm{CFI}-400945$ for 48 hours. Genomic DNA from the cells was spotted onto a nitrocellulose membrane with or without prior treatment with RNaseH1. The dots were stained with an $\$ 9.6$ antibody to detect RNA: DNA hybrids. For normalization, an antibody against single-stranded DNA (ssDNA) was used to stain an additional set of dots that had been treated with acid for denaturation.

(E) Quantification of the S9.6 signal on the membrane from (D). The signal obtained with S9.6 was normalized to the ssDNA signal first and then to the control treatment. Each column represents the average of three biological replicates with three technical replicates each.

(F) Dot-blot analysis of RNA: DNA hybrids in synchronized H1299 cells. Cells were reverse transfected with pooled siRNA for 72 hours, followed by Dot-blot analysis as described in (D).

(G) Quantification of the S9.6 signal on the membrane as described in (E). The signal obtained with the S9.6 antibody was normalized to the ssDNA signal first and then to the control treatment. Each column represents the average of three biological replicates with three technical replicates each.

(H) Immunostaining of the R-loop signal in cell nuclei upon treatment with Centrinone B for 48 hours and $10 \mu \mathrm{M}$ MK2i for 24 hours. The nuclear signal was visualized by staining the cells with S9.6 antibody and 4', 6-Diamidin-2-phenylindol (DAPI) was used to delineate the nuclei (scale bar $=20 \mu \mathrm{m}$ ).

(I) Quantification of the R-loop signal in cell nuclei upon treatment with Centrinone B for 48 hours, followed by treating the cells with $10 \mu \mathrm{M}$ MK2illl for 24 hours. The nuclear signal was quantified using the ImageJ software. The mean and distribution of three biological replicates (integrated) were calculated, and the significance was assessed using the Mann-Whitney test.

(J) Representative dot blot showing the rescuing of accumulation of RNA: DNA hybrids upon MLK3/MK2 inhibition.

(K) Quantification of the S9.6 signal on the membrane corresponding to dot blot in (J). The signal obtained with S9.6 was normalized to the ssDNA signal first and then to the control treatment. Each column represents the average of three biological replicates with three technical replicates each.

(L) Validation for RNAseH1 overexpression in synchronized H1299 cells.

(M) The resolution of RNA: DNA hybrids by RNAseH1 rescues DNA replication fork progression upon Centrinone $B$ treatment. Cells were transfected with plasmids as described in (B). DNA fiber assays were performed to determine replication fork progression. 


\subsection{PLK4 inhibition leads to activation of the transcription factors ATF2 and JUN, downstream of the p38/MK2 pathway}

During the process of DNA synthesis and in every entry into S-phase, the DNA replication machinery is threatened by multiple intrinsic factors that can lead to DNA damage and genomic instability, such as lesions that interfere with fork progression, tightly associated DNA-protein complexes, and transcription-replication conflicts (65). Therefore, cells must overcome these obstacles to ensure faithful DNA replication and chromosome duplication. However, if the cell fails to overcome these barriers, this will eventually lead to genomic instability and reduced cell viability, a hallmark of cancer, and aging. Both DNA replication and transcription are vital cellular processes. Both types of machinery are competing over the same DNA template, which increases the chance of the two types of mechanisms colliding with each other under certain circumstances.

For the reasons mentioned above, we next searched for a mechanism that may drive unscheduled transcription and lead to the formation of RNA: DNA hybrids. To investigate whether global transcription is indeed responsible for compromised DNA replication, we used an inhibitor of Cdk9 to shut down transcription, as described previously $(78,104)$, exploring potential transcription factors downstream of $\mathrm{p} 38 / \mathrm{MK} 2$ signaling that may play a role in our scenario. Upon activation of p38 and MK2, the transcription factors ATF2 and JUN (also known as c-Jun) are phosphorylated and form a dimer to stimulate transcription $(82,103,130)$. ATF2 is a member of the leucine zipper family of DNA binding proteins and is known for its ability to form a homodimer or a heterodimer with c-Jun, which further stimulates transcription (32-34).

Accordingly, we detected an increase in the phosphorylation levels of ATF2 and JUN upon Centrinone B treatment (Figure 6.8 A). In this context, ATF2/JUN phosphorylation was dependent on MK2 and MLK3 (Figure 6.8 C). At the same time, we observed the accumulation of both transcription factors in the chromatin fraction (Figure 6.8 B). While CDK9 inhibition rescued DNA replication in the presence of the PLK4 inhibitor Centrinone B (Figure 6.8 F), JUN depletion also restored DNA replication when PLK4 was inhibited (Figure 6.8 D, E). Since JUN-mediated transcription affects a multitude of genes, possibly leading to unscheduled RNA accumulation, we hypothesized that JUN activation might contribute to the observed replication stress but not only.

To clarify the relationship between the activation of ATF2/JUN and replication stress, we performed DNA fiber assays after depleting JUN and treating the cells with Centrinone B. To test whether JUN is the main reason for transcription activation, we carried out dot blot analysis. This revealed that JUN depletion partially rescues $\mathrm{R}$-loop formation while blocking transcription with CDK9 
inhibitor rescues it completely, indicating that JUN is part of this activation but not the only one (Figure $6.8 \mathrm{G}, \mathrm{H}$ ). We conclude that interfering with centrosome integrity leads to replication stress partially by JUN-mediated transcription.

A
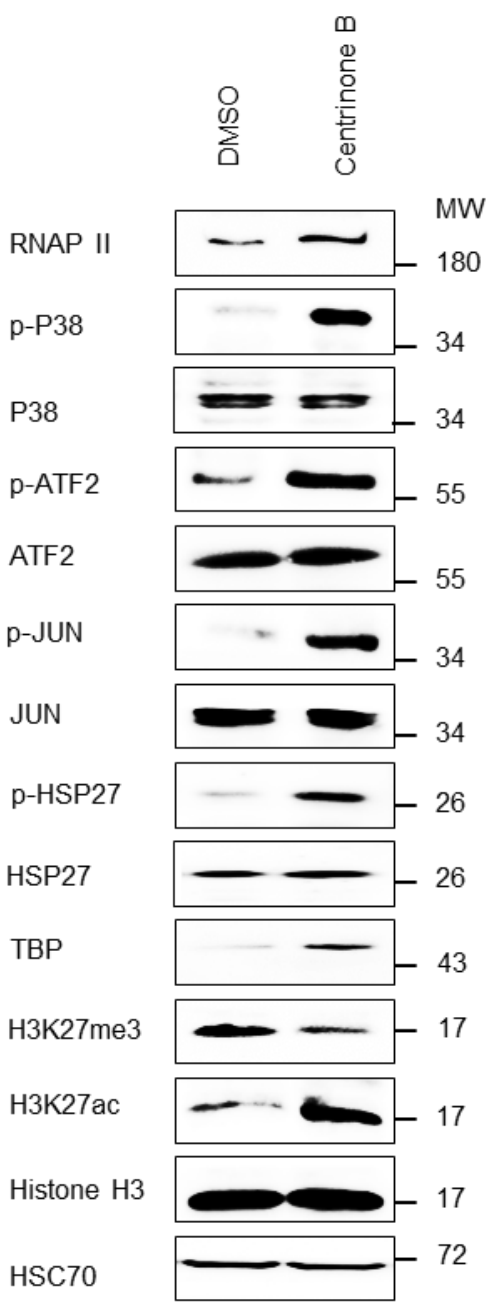

B

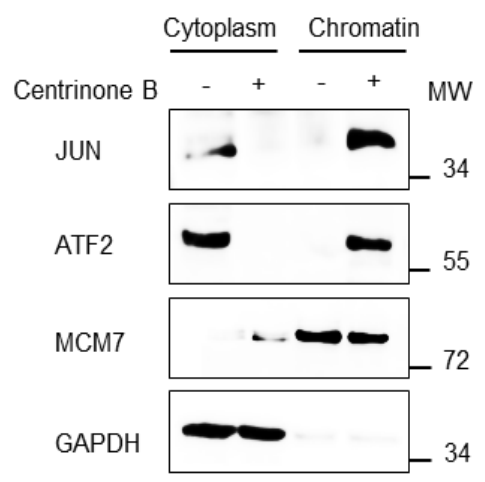

C

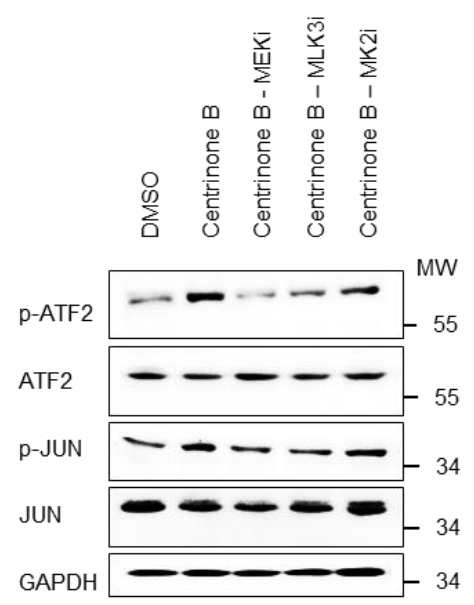


D

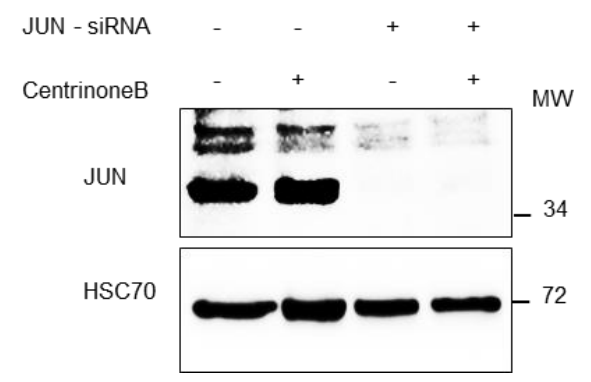

F

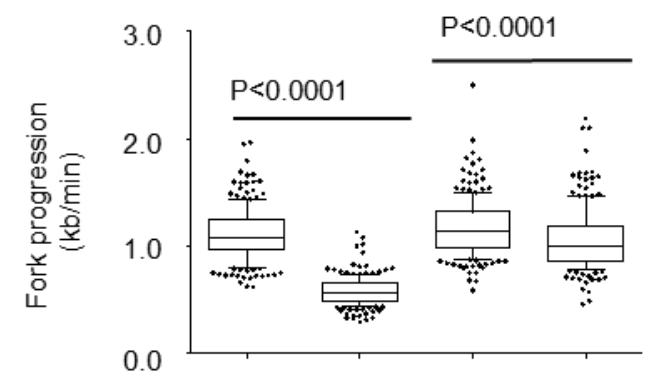

Centrinone B

CDK9i

H

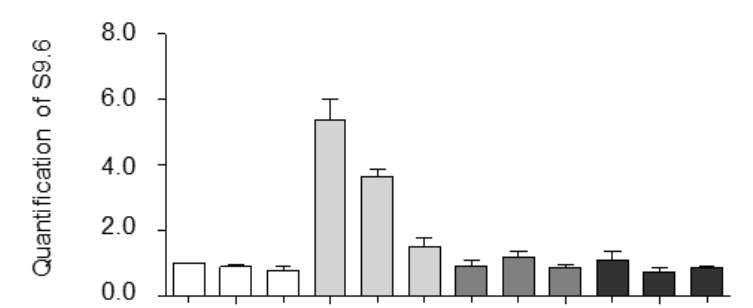

Control siRNA

JUN SiRNA

CDK9i

DMSO

Centrinone B

RNaseH1
E

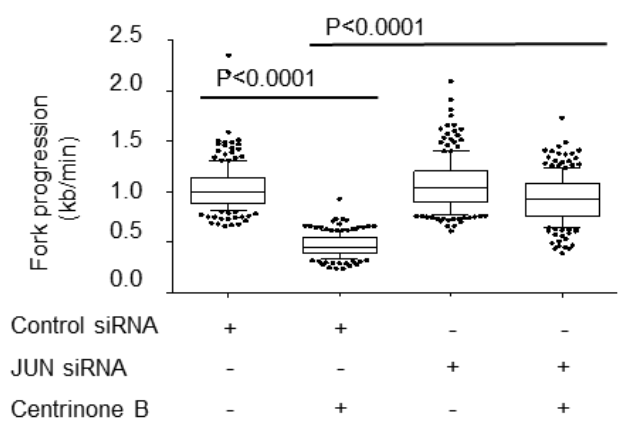

G

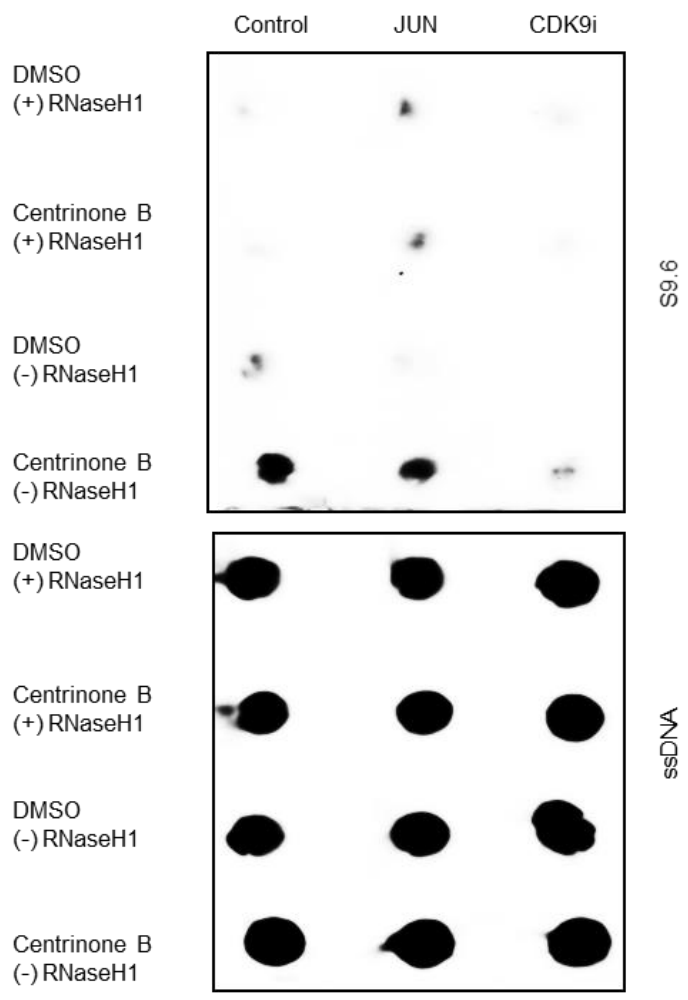

Figure 6.8: PLK4 inhibition activates ATF2 and JUN to induce replication stress.

(A) Phosphorylation of ATF2 and JUN after Centrinone B treatment ( $500 \mathrm{nM}, 48$ hours) revealed by immunoblot analysis. Note the additional accumulation of TBP, the reduction in the repressive Histone 3 trimethylation at K27, and the increase in H3K27 acetylation, all of which in agreement with the increased transcriptional activity. 
(B) JUN and ATF2 associate with the chromatin fraction upon Centrinone B treatment. Chromatin fractions were isolated and compared to the cytoplasmic supernatant. MCM7 (chromatin) and GAPDH (cytoplasm) were used to control the fractionation. The gel is a representative example of 3 biological replicates.

(C) ATF2/JUN phosphorylation is dependent on the activity of MLK3 and MK2. H1299 cells were treated as described in (A) in addition to MLK3i, MK2illl, and MEKi (U0126). The phosphorylation levels of ATF2 and JUN were assessed using immunoblot analysis.

(D) Western blot analysis showing the validation of JUN knocked down after 72 hours.

(E) The impairment of DNA fork progression upon Centrinone B treatment is dependent on JUN. Synchronized H1299 cells were reverse transfected to knock down JUN and treated with $500 \mathrm{nM}$ Centrinone B for 48 hours. DNA fiber assays determined fork progression.

(F) Blocking the global transcription machinery using a Cdk9 inhibitor rescues DNA fork progression upon PLK4 inhibition. Synchronized H1299 cells were treated with either DMSO or $500 \mathrm{nM}$ Centrinone B for 48 hours, and 2 hours before harvesting, the cells were treated with $10 \mu \mathrm{M}$ Cdk9 inhibitor. DNA fiber assays were performed to assess fork progression.

(G) Dot blot analysis of synchronized H1299 cells treated as indicated underneath the figure. Genomic DNA from the cells was spotted onto a nitrocellulose membrane with or without prior treatment with RNaseH1. The dots were stained with the S9.6 antibody to detect RNA: DNA hybrids. For normalization, an antibody against single-stranded DNA (ssDNA) was used to stain an additional set of dots that had been treated with acid for denaturation.

(H) Quantification of the S9.6 signal from Dot blot in (G). The signal obtained with S9.6 was normalized to the ssDNA signal first and then to the control treatment. Each column represents the average of three biological replicates with three technical replicates each.

\subsection{MK2 inhibition rescues defects in replication and proliferation of cells from patients with Seckel syndrome}

Seckel syndrome an autosomal recessive disorder displayed by growth retardation, microcephaly, minimal head, intellectual disability, and unique facial features, such as large eyes, beak-like nose, and narrow face. Seckel syndrome can be caused by mutations in several centrosomal components, carry out by nine components such as have been described $(32,51)$. The initial suspicion that centrosomes might govern DNA replication had come from the fact that genetic defects of centrosomes on the one hand and the replication stress kinase ATR, on the other hand, each lead to highly overlapping phenotypes in Seckel syndrome $(32,112)$. Therefore, we asked whether the cells from patients suffering from a centrosomal defect causing Seckel syndrome might also display the features of replication stress. Indeed, human fibroblasts from a patient with Seckel syndrome with a defect in the centrosomal component CEP152 (32) showed a substantially slower replication fork progression than fibroblasts from healthy donors (Figure 6.9 A).

Moreover, the Seckel cells had increased MK2 activity, as determined by the phosphorylation of the corresponding signaling intermediate (Figure 6.9 B). Strikingly, MK2 inhibition rescued the progression of replication forks in Seckel cells despite the accumulation of P53, which can be due to the rescue of the p21 level after MK2 inhibition (Figure $6.9 \mathrm{C}$ ). p38/MK2 has long been known to 
mediate the phosphorylation of p53 at Ser20 (135) and may thus alter its activity even when its levels are unchanged. Moreover, although Seckel fibroblasts grew substantially more slowly than normal fibroblasts, incubation with MK2 inhibitor led to equally efficient growth of all cells alike (Figure 6.9 D). Thus, centrosome disintegration in patients with Seckel syndrome leads to MK2 activation and replication stress as a consequence. The fact that MK2 inhibition restores DNA replication and proliferation in these cells might suggest a potential treatment option that needs further evaluation before implementation.

A

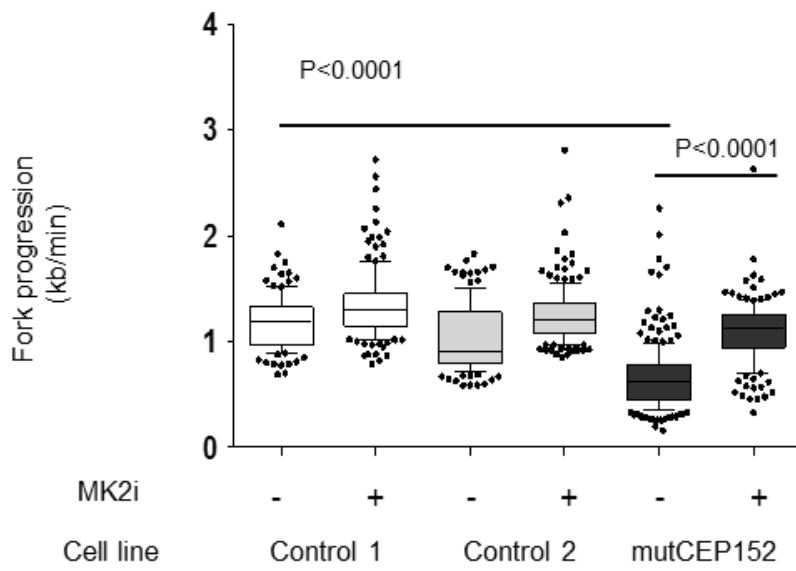

C

Control 1 Control 2 mutCEP152

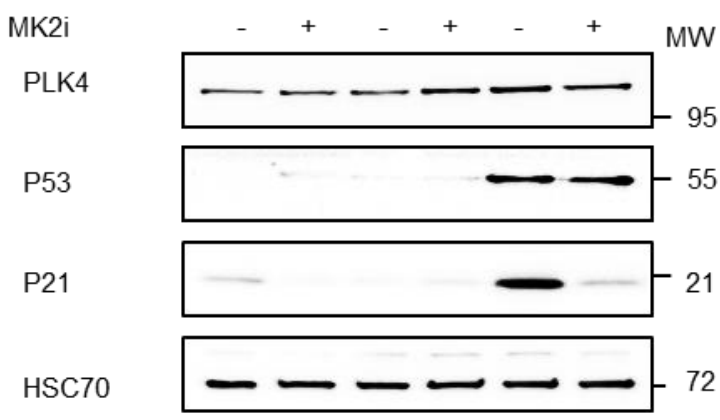

B

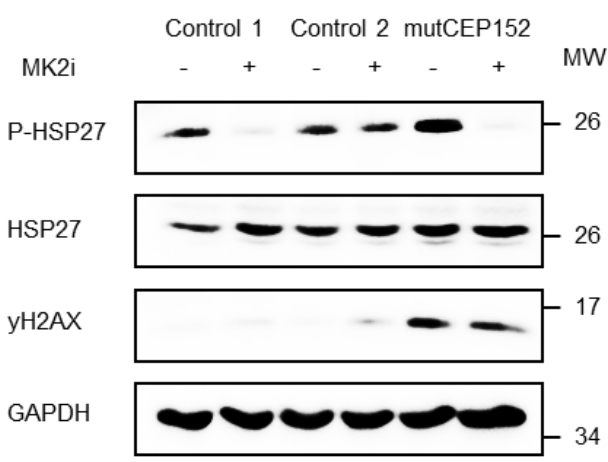

D

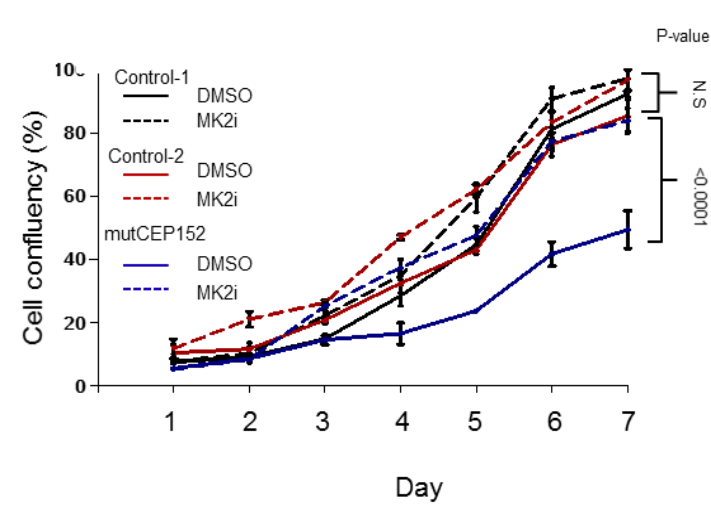

Figure 6.9: MK2 inhibition facilitates DNA replication and proliferation of cells derived from a patient with Seckel syndrome.

(A) The MK2 inhibitor MK2i III rescues DNA replication fork progression in cells from a Seckel patient carrying a deletion in the CEP152 gene. Human breast fibroblasts (control-1), human skin fibroblasts (control-2), and skin fibroblasts from a Seckel patient (CEP152 mutant) were treated with $20 \mu \mathrm{M}$ MK2illl for 48 hours, followed by fiber assays.

(B) The MLK3-p38-MK2 pathway was constitutively activated in Seckel syndrome cells. Control-1, control-2, and Seckel cells (deletion in CEP152) were harvested after 48 hours of $20 \mu \mathrm{M}$ MK2i III treatment and subjected to immunoblot analysis.

(C) MK2 inhibition rescues p21 levels in Seckel syndrome cells despite the continuous activation of p53. 
(D) The restored proliferation rate of Seckel cells upon MK2 inhibition. $5^{\star} 10^{3}$ control-1, control-2, and Seckel cells were seeded in wells of a 24-well plate and treated with either DMSO or $10 \mu \mathrm{M}$ MK2i III. A Celigo Cytometer was used to determine cell proliferation. The average of six technical replicates per three biologicals replicates was summarized at each time point. 


\section{DISCUSSION}

\section{DISCUSSION}

In this thesis, we demonstrate a direct connection between DNA replication and centrosome integrity during S phase. Strikingly, centrosome disruption leads to an impairment of DNA replication even when cells are not allowed to undergo mitosis. Centrosomes support DNA replication by regulating the MLK3-p38-MK2 signalling pathway as well as suppressing the formation of RNA:DNA hybrids (R-loops). Based on that, this thesis determines a mechanistic link between the duplications of two major elements, which need to be duplicated once and only once every cell cycle - the genome and the centrosomes.

\subsection{The depletion of centrosomal components reduces DNA replication fork progression in H1299 cells}

Centrosomes serve as the microtubule-organizing centers and contribute to cell division. They consist of two centrioles, each one is being surrounded by multiple proteins $(6,8)$. These proteins are responsible for various functions, such as centrosome duplication, microtubule nucleation, and elongation. Centrosome abnormalities have been observed in several cancer types. Defects, such as centrosome amplification, are associated with poor prognosis, metastasis, aggressiveness, and drug resistance $(6,8)$. Therefore, for the last few years, there has been an increasing interest in studying centrosomes, especially in cancer. Understanding the biology behind centrosomal abnormalities can help us to find a suitable way to target these organelles to achieve a better survival outcome. Investigating how centrosome abnormalities promote tumorigenesis is not a new field of research. A century ago, Theodor Boveri proposed that increased centrosome numbers are a pro-tumorigenic driver. Years after Boveri's hypothesis, cancer biologists included centrosome amplification to the hallmarks of cancer (3).

Centrosome duplication is a crucial step in centrosome biogenesis, much like DNA replication, and it is tightly regulated to ensure the one-time duplication every cell cycle. The process of centrosome duplication at G1 phase starts with the recruitment of both CDK2 and PLK4 to centrosomes to initiate the duplication process. The recruitment process of CDK2 and PLK4 is carried out by the scaffold proteins CEP152 and CEP192 (10, 11). The loss of CEP152 has been reported to cause a reduction in the recruitment of PCM proteins required for centriole 


\section{DISCUSSION}

duplication and, therefore, failure in centriole duplication and loss of centrioles. In addition to that, loss of CEP152 contributes to further delay in the recruitment of SAS6 to centrosomes, impairing centrosome duplication, and driving the formation of monopolar mitotic spindles during mitosis $(10,11)$. Similar to what is known in the filed of centrosomes biogenesis, in our experiments, we observed a significant reduction in the detectable centrosomes (Pericentrin) upon CEP152, CEP192 knock down, as well as SASS6 and CCP110 knock down(Figure 6.1).

CEP192 is another centrosomal component that we investigated in our study. It is essential for the recruitment of pericentriolar material (PCM) and plays a role in centrosome maturation and duplication (11). Finally, CCP110 is an additional centrosomal protein required for centrosome duplication but at a different stage of pro-centriole formation. CCP110 caps the distal tips of pre-existing centrioles, and by doing this, along with CEP97, it controls the length of procentrioles by polymerization and de-polymerization of y-tubulin (16). CCP110 is also required for correct spindle formation, regulation of cytokinesis, and, therefore, genomic stability (137). Previously, it has been shown that depletion of different centrosomal components could lead to different alterations of centrosomes. For example, the group of Chen found that loss of CCP110 results in premature centrosome separation, and abolishes centrosome reduplication in S-phasearrested cells (137).

As previously stated by Le Clech et al. 2009 (16), depleting SASS6 weaken centrosome duplication by impairing the growth of the procentriole (138). In agreement with previous knowledge, we also observed a defect in centriole duplication in all of the cases. We also tried to detect any significant differences in the morphology of the centrosomes, but detecting such differences might require higher resolution than the one available by standard microscopy. In addition to the reduction of centrosomes, we also observed the induction of replication stress when depleting these components. Thereby, we can conclude that the various phenotypes induced by the knockdowns overlap but are distinct. Yet what they all have in common is the defects in the duplication of centrioles and the induction of replicative stress.

Targeting centrosomes might represent a plausible way to treat cancers that exhibit centrosome amplification. As PLK4 is one of the leading players in centrosome duplication, and it is not only responsible for the initiation of duplication but also responsible for maintaining the correct centrosome number within the cell, therefore developing drugs to target PLK4 would be one way to target centrosomal amplification in cancer. The process of centrosome duplication is 


\section{DISCUSSION}

triggered by the recruitment of PLK4 to the centrosomal site leading to the interaction between the recruited PLK4 and STIL. the interaction between PLK4-STIL induces the autophosphorylation of PLK4 to activate STIL. The phosphorylation of STIL by PLK4 triggers the recruitment of SAS-6 and cartwheel assembly to ensure the 9-fold symmetry $(1,2$, and 8$)$. While the inhibition of PLK4 leads to aberrant centriole duplication during the early stages of the cell cycle G1/S phases $(14,15)$, the overexpression of PLK4 leads to centrosome amplification through the simultaneous generation of multiple pro-centrioles adjoining to each parental centriole during the $S$ phase (83). PLK4 is not only required during the early stages of the cycle (G1/S phase), but it seems like it is also needed during the later stages to maintain the integrity of centriolar satellites, as previously reported $(140,141)$. PLK4 is also required for the phosphorylation of CDC25C and CHK2, which regulate the entry into mitosis $(30,83)$. Consistent with the important role of PLK4 in controlling centriole duplication, its activity as well as its level need to be tightly regulated. For this reason, PLK4 represents a possible target for cancer therapy. Since 2013 several PLK4 inhibitors have been developed to serve this purpose, one of which sucessed to enter the clinical trial; the PLK4 inhibitor CFI-400945. CFI-400945 showed high efficacy in animal models of breast and ovarian cancers. It was further tested in patients with advanced tumors in phase I clinical trials and is currently being tested in phase II clinical trials in patients with advanced/metastatic breast cancer (ClinicalTrials.gov Identifier: NCT03624543, 2018). Pre-clinical trials with CFI-400945 have shown that inhibiting PLK4 causes aberrant chromosome numbers, which in turn leads to mitotic catastrophe and cell death $(81,82)$. PLK4 inhibition using CFI-400945 shrinks the size of the tumor in animals and exhibits anti-tumor activity in patients with low side effects (82).

Despite the impressive outcomes of CFI-400945, it is essential to keep in mind that this small compound promotes mitotic catastrophe and cell death through centrosome amplification (81). Whether the observed effect is due to targeting PLK4 or Aurora kinases or both is still unclear. Since centrosome amplification drives tumorigenesis, some legitimate concerns need to be addressed regarding the CFI-400945 inhibitor. For example, what would happen to the cells, which manage to evade the mitotic catastrophe caused by CFI-400945? Do these cells become more resistant to other treatments, thus becoming more aggressive? To answer these questions, further studies should be carried out on CFI-400945.

In 2015, (108) another PLK4 inhibitor called Centrinone B was described. Centrinone B is a selective PLK4 inhibitor that has been reported to deplete centrioles in human and other 


\section{DISCUSSION}

vertebrate cell types. Similar to what was previously described (108), the inhibition of PLK4 by Centrinone B reduces the total number of centrosomes in $\mathrm{H} 1299$ and other cell lines. Additionally, we also showed that the status of p53 had no significat impact on centrosome number nor DNA fork progression upon PLK4 inhibition, suggesting that PLK4 is highly required for centrosome duplication in different cancer types .

In dividing cells, centrosome integrity can influence the rate of cell division $(81,82,108)$. We observed that inhibition of PLK4 reduced cellular growth, but not only, it also managed to induce DNA replication stress in several human cancer cell lines. While the observed effect appears to be a direct effect of Centrinone B on PLK4, the exogenous overexpression of PLK4 in H1299 rescued the DNA replication fork progression and increased resistance towards gemcitabine (Figure 6.3), suggesting a potent pro-proliferative function of PLK4.

In summary, the increase in PLK4 expression was found in medulloblastoma, breast, colorectal, prostate, and ovarian cancers (83), which motivated many scientists to investigate PLK4 as a target for cancer therapy. Even though only one PLK4 inhibitor managed to make it to clinical trial phase II, this had opened the way to the development of similar centrosomal inhibitors. Moreover, it might be worthwhile to test other centrosomal biomarkers, which can be used as a target for cancer therapy in addition to PLK4 (13). In vitro studies indeed showed that overexpression of SAS6 (12), STIL (13) and pericentrin (13) could also lead to centrosome overduplication, but this needs to be further explored.

\subsection{PLK4 inhibition drives cells towards polyploidy}

Aneuploidy, or imbalanced chromosome number, has been studied for decades and was proven to be a mechanism that profoundly affects cell functions. Moreover, aneuploidy is very frequent in cancer, and it is often associated with a more complex phenotype called chromosomal instability (CIN). It is also known that chromosomal instability and aneuploidy in cancer correlate with resistance to drugs, metastasis, and disease progression (107).

Several mechanisms can lead to aneuploidy: firstly, chromosome segregation errors, which result from incorrect attachments of the spindle microtubules to the kinetochore; secondly, oncogenic activation and/or tumor suppressor inactivation; thirdly, the fidelity of chromosome segregation can be influenced by changes that occurred at the transcriptional level as in the case 


\section{DISCUSSION}

of Rb-E2F and Ras activation (107). Accordingly, the gain of a single extra chromosome can strongly affect cell proliferation.

Centrosome abnormalities known to be correlated with chromosomal instability (CIN), such as; insufficient levels of PLK4 known to be associated with abnormal spindle poles (4). However, it is still unknown to what extent CIN results from a direct impact on the spindle and, most importantly, to what extent DNA replication stress is involved. What would also be interesting to address is what comes first, CIN or DNA replicative stress, thus, is CIN driving DNA replicative stress or vice versa?

Chromosomal instability (CIN) is known to be a source of replicative stress (113). Therefore, in continuously cycling cells, we cannot rule out the possibility that the observed reduction in fork progression could be a result of chromosomal missegregation rather than a direct effect on the DNA replication machinery, especially when all of our treatment timelines are between 48 and 72 hours. Therefore, we tested when and to what extent centrosomal depletion through PLK4 inhibition can induce aneuploidy using chromosomal spreading and FACS analysis.

We have shown that the PLK4 inhibitor Centrinone B reduces cell proliferation and centrosome number, which is in agreement with what was previously described (108). Additionally, we also showed that PLK4 inhibition impaired the progression of the replication fork. Using both chromosomal spreading and FACS analysis, we observed an increase in polyploidy upon Centrinone B treatment only after seven days of treatment. Thus, we can safely argue that the reduction in fork progression is not a result of aneuploidy but rather a result of constant interfering with the DNA replication machinery.

\subsection{Centrosome integrity is essential to avoid DNA replication stress even independently of mitosis}

Centrosomes function as the microtubule-organizing center in interphase and mitotic cells. In addition, they might also play a role in the regulation of cell cycle progression itself, since laser ablation or surgical removal of centrioles lead to failure in cytokinesis and G1 arrest, despite the observed slower cell cycle progression (84). Similarly, we have also shown that cells with knocked down centrosome components or inhibited PLK4 also experienced a slower cell division (Figure 6.3-5). Therefore, we can conclude that there might be a sort of mutual coordination between 


\section{DISCUSSION}

centrosome duplication and DNA replication. Multiple evidence could support this coordination, such as the activation and inactivation of the serine/threonine cyclin-dependent protein kinases (CDKs) (85), e.g., CDK1 and cyclin B. CDK2 activation is required not only for rapid DNA replication (87), but it has also been shown to be essential for the activity of centrosomes during the G2/M transition, as demonstrated by Bailly et al. (86), and later on the importance of CDK2 in controlling centrosomes duplication was also revealed(84). Moreover, CDK2 activity is a crucial regulator of the centrosome cycle, such as centrosomes duplication (11, 12, and 26) and separation of the centriole pair $(13,80)$. However, the signaling pathways involved in controlling the initial activation of CDK1/2 at the centrosome remain enigmatic.

Both centrosome duplication and DNA replication are controlled by the retinoblastoma tumor suppressor pathway, a pathway that governs the expression of the transcription factor E2F, which is responsible for $S$ phase progression $(142,143)$. Inhibiting DNA replication using hydroxyurea (HU) led to centrosome amplification in $\mathrm{pRb}$ deficient human and mouse fibroblasts (144), but also interfering with centrosomes causes replication stress throughmalfunctioning of the mitotic spindle (89), indicating that the centrosome cycle could be partially dependent on DNA replication $(88,144)$. When centrosomes disintegrate, mitotic fidelity can be decreased, thus enhancing the missegregation of sister chromatids, leading to numerical chromosomal instability $(\mathrm{CIN})$, which would then result in replication stress, as recently observed in cells with supernumerary chromosomes (113). Therefore, in continuously cycling cells, we cannot rule out such a scenario even though we could not observe aneuploidy before the seventh day of treatment, yet having lagging or broken chromosomes is known to be sufficient to induce replicative stress.

Our previous results using chromosomal spreading analysis indicate that Centrinone B induces aneuploidy after seven days of treatment. Therefore, we aimed to prevent the cells from entering the cell cycle under treatment to further avoid the consequences which may arise from the $\mathrm{M}$ phase in dividing cells. Moreover, as one of our main aims in this study, we sought to investigate whether the impairment of centrosome components has a direct impact on the DNA replication machinery independently from chromosome missegregation (89).

To address this question, we arrested the cells in G1 phase for 48 hours using a CDK4/6 inhibitor during siRNA transfection or PLK4 inhibitor treatment (Figure 6.4). Using this system, we tested whether we can still observe the same effect on the replication fork progression. 


\section{DISCUSSION}

Interestingly, centrosome disruption always leads to replication stress even without any passage through mitosis (Figure 6.4), which remained true across all treatments. Our results indicate that centrosomes might not be strictly required for mitotic spindle assembly but instead have other functions, such as maintaining the processivity of DNA replication independently of mitosis and apart from polyploidy. The role of centrosomes in mitosis is a topic that is still under debate. It has become evident that centrosomes are not strictly required for mitotic division in all cell types, but instead, they contribute to the accuracy of mitosis in some cell types (7). It is thus very intriguing to hypothesize that the role of centrosomes in DNA replication might be at least as necessary as their contribution to the accuracy of mitotic cell division.

\subsection{Depletion of centrosomal components triggers the p38/MK2 signaling pathway, leading to replication stress}

Several checkpoints control the G1/S and G2/M transitions to ensure a faithful cell division (90). They are also associated with centrosomes and/or appear to play an essential role in centrosome homeostasis $(91,92)$. For instance, the tumor suppressor p53 controls both G1/S and G2/M checkpoints, and its inactivation leads to dysregulation of the centrosome cycle (93). In human cancers, mutations in p53 correlate with the occurrence of centrosome amplification in carcinomas of the breast, head and neck, prostate, and in neuroectodermal tumors (94). In cultured cells, tumors derived from p53-null and p53 wild-type mice, which are associated with cyclin E overexpression synergistically, increased the frequency of centrosome amplification (95). These previous findings suggest that an imbalance between negative and positive cell cycle regulators could accelerate centrosome defects.

In this project, we aimed to search for a plausible mechanism that might impair DNA replication fork progression upon centrosome disintegration. We have previously characterized the role of MK2 in replication stress. In response to irradiation, treatment with gemcitabine, or CHK1 inhibition, the resulting replication stress highly depends on MK2 activity. At least in part, the rescue of fork progression after MK2 inhibition/depletion was a result of the reactivation of translesion synthesis polymerases, and accordingly, DNA polymerase eta was shown to be a substrate of MK2 (114). 


\section{DISCUSSION}

Indeed, the phosphorylated and thus, the active forms of p38 and MK2 were strongly enhanced by PLK4 inhibition (Figure 6.5), and the same was found for the bona fide MK2 substrate HSP27. Thus, disruption of centrosomes activates p38/MK2 signaling independently of mitotic insults. The available pieces of literature show that p38 can be triggered by centrosomes impairment, followed by activation of p53-p21-dependent G1-S arrest and that the activation of p38 is vital for this activation (126). Moreover, the Suhail et al. reported that the depletion of TACC3, a critical centrosomal protein that is up-regulated in many cancers, lead to G1 arrest and cell death through activation of the p38-p53-p21 stress signaling pathway (145). Similarly, we found that depleting other centrosomal components, such as PLK4, CEP152, and SAS6, also lead to the activation of p38/MK2 pathway and impairment of the replication fork. Altogether, we suggest that this signaling pathway is triggered by centrosome disruption, resulting in diminished progression of DNA synthesis.

\subsection{MLK3 acts as the first sensor towards impairment of centrosomes, followed by activation of the p38/MK2 pathway}

Several studies showed that multiple proteins could influence both mechanisms centrosome duplication $(79,126)$ and DNA replication $(39,40$, and 41). The Cyclin E/Cdk2 complex is known to regulate both DNA replication and centrosome duplication during the G1/S phase of the cell cycle (98). It was also shown that the initiation of DNA replication requires the placement of Cyclin E/Cdk2 to the centrosomes site by a domain of 20 amino acids known as centrosomal localization sequence (CLS) (98).

CHK1 kinase was also suggested to be associated with centrosomes in interphase rather than in the mitotic phase. CHK1 negatively regulates entry into mitosis by inhibiting the activation of cyclin B/CDK1 under abnormal cell conditions. The kinase works as a shield towards centrosomal CDK1 by protecting it from unscheduled activation, thereby contributing to the proper timing of the initial steps of cell division, including mitotic spindle formation (99).

Searching for potential candidates which can explain the activation of P38/MK2 upon centrosomes impairment and in response to that inducing replicative stress, we investigated the mixed-lineage kinases 3 (MLK3). MLK3 is a MAP3K11 kinase known for its ability to activate MKK3/6 /c-JUN/JNK/ERK $(100,115)$ as well as for its association with centrosomes (116). It has been previously shown that MLK3 can function as a scaffold protein, as in the case of RAF1/ERK 


\section{DISCUSSION}

MAPK3K BRAF, by bridging them together, leading to the activation of RAF-1 $(117,118)$. Moreover, MLK3 activation was mostly enhanced during G2/M to regulate the microtubule organization during mitosis in transformed HeLa cells, while inhibiting MLK3 using CEP-11004 managed to block mitotic progression and caused cells to arrest in pro-metaphase (146). Furthermore, MLK3 expression has also been reported to promote activation of the MAPK p38 pathway, NF-KB (100), and p70 S6 kinase (101). On the other hand, depleting this kinase was neither toxic to cells, nor essential for progression through the cell cycle, but its activity was required to increase the sensitivity towards taxol compounds (147).

We tested whether centrosomal MLK3 could play a role in the regulation between DNA replication and centrosome duplication, independently of mitosis. In agreement with all previous findings, we observed MLK3 co-localization to the site of centrosomes during $S$ phase. We also found a high dependency on MLK3 to activate p38/MK2, suggesting a close regulatory association of the three kinases. One function of this complex is the transmission of a signal that connects the replication of centrosomes and the cellular DNA. It was also known that MLK3 could localize to centrosomes, and it appears to regulate microtubule organization during mitosis in a JNK-independent fashion (146). MLK3 can interact with several scaffold proteins known as JNK interacting proteins (JIPS), JIP1, JIP2, and JIP3 to relocate themselves inside the cell. In addition to that, JIPs are recognized as the cargos for the molecular motor kinesin, the motor protein that moves along microtubule filaments. The association of MLK3 with JIP may provide further explanation of the dynamic cellular distribution of $\operatorname{MLK} 3(100,101)$.

Our results indicate that there might be an increased cancer risk associated with targeting MLK3 since centrosome malfunction would no longer be sensed through MLK3 activation and replication stress. This possibility should be evaluated in animal cancer models before taking the approach to patients. In summary, we suggest that the initial steps of an accurate DNA replication require highly intact centrosomes at the G1/S transition and that MLK3 works as a guardian of centrosomes. 


\section{DISCUSSION}

\subsection{Centrosome disintegration induces RNA:DNA hybrids, an additional source of DNA replication stress, in part through the activation of the transcription factor ATF2/JUN downstream of p38/MK2}

Based on the activation of p38/MK2 via centrosomal MLK3 activity upon centrosome impairment and the potential involvement of the JUN/ERK pathway (149), a pathway known to strongly activate several transcription genes (149), we, decided to test whether the transcriptional machinery was activated due to centrosomes impairment. It is known that upon activation of p38, several transcription factors are being activated, such as ATF2, which is a transcription factor activated by several stress kinases including JNK (MAPK8, MAPK9, MAPK10) and p38 (MAPK1, MAPK11, MAPK12, MAPK13, MAPK14) (148). It was perviously shown that ATF2 can affect the transcriptional regulation of early genes regulating stress responses and DNA damage responses $(102,148)$. Upon activation of ATF2 by different stress stimuli, ATF2 forms homodimers or a heterodimer with JUN (103), inducing the translocation of the complex to the nucleus. ATF2 is known to be phosphorylated on threonine 69 and/or 71 by JNK or by p38 in response to stimuli or by ATM on serines 490 and 498 (150). On the one hand, the phosphorylation on Thr69 and Thr71 of ATF2 and its dimerization are required to induce its transcription factor activity, which further activates the transcriptional activator protein 1 (AP-1). The phosphorylation on serines 490 and 498 revealed another role for ATF2 as a participant in the DNA damage response (151). We confirmed the activation of the transcription machinery by screening for transcriptional markers, such as H3K27ac and H3K27me3, and we also observed the activation of ATF2 and JUN (Figure 6.7). In addition, we showed the translocation of these transcription factors ATF2/JUN to the nucleus, as well as their dependency on active MLK3. Considering all these findings, in which centrosomes impairment present an additional way to activate transcription machinery, we sought to test whether the observed imbalance in transcription levels could lead to a conflict with the DNA replication machinery through the generation of R-loops.

Conflicts between transcription and DNA replication represent a significant cause of replication stress (104). Several mechanisms have been suggested for R-loops forming obstacles to the DNA replication machinery $(60,104)$. Such conflicts may not merely result from collisions between RNA- and DNA-polymerases, but rather from the co-transcriptional occurrence of RNA:DNA hybrids with an additional single DNA strand, the so-called R-loop. It was shown that R-loops hinder DNA replication forks from progressing (60), leading to the assumption that Rloops are an additional source of stress. We tested whether R-loops could be a potential 


\section{DISCUSSION}

explanation for the observed reduction in fork progression through the activation of transcription machinery upon impairment of centrosomes.

Thus, we tested whether R-loop formation generated due to the activation of transcription factors might be implicated upon centrosomes impairment. Notably, our results strongly suggest that depletion of PLK4 induces R-loops and therefore interferes with DNA replication (Figure 6.6), whereas DNA replication could be restored by RNase-mediated removal of R-loops. Moreover, the observed accumulation of R-loops was dependent on the activity of MLK3/MK2.

RNA metabolism is becoming a significant focus in current research on DNA replication stress. RNA processing factors constitute a substantial fraction of kinase substrates upon DNA damage (105), making it plausible that such factors might affect DNA integrity, perhaps through R-loop formation. Therefore, targeting centrosomal components in cancer cells is expected to have a more profound impact on cancer cell proliferation by interfering with DNA replication through transcription-replication conflicts. The raised conflict by itself encourages the continuous evaluation of PLK4 as a drug target.

\subsection{MK2 inhibition rescues defects in DNA replication and proliferation of Seckel syndrome patient cells}

Seckel syndrome is a genetically heterogeneous disorder that can be caused by a mutation in mainly ten genes, including ATR, RBBP8, CEP152, CENPJ, PLK4, CEP63, DNA2, ATRIP, NIN and CDK5RAP2 (109). The syndrome is characterized by growth retardation, microcephaly, reduced head circumference, intellectual disability, and unique facial features, such as large eyes, beak-like nose, and narrow face. This phenotype resembling human Seckel syndrome, including primary microcephaly, was also found in mice with a targeted deletion of CEP63 (52). Classically, both the human Seckel syndrome and its murine model were described in response to hypomorphic recessive alleles of ATR, the central mediator of the replication stress response, which is activated upon replication stress (49).

Several studies showed the involvement of Seckel syndrome-related genes in various cellular processes, such as centrosome duplication and maturation (CEP152, CENPJ, CEP63, 


\section{DISCUSSION}

NIN, CDK5RAP2, and PLK4), while some others (ATR, ATRIP, RBBP8, and DNA2) are important in maintaining genomic stability.

PLK4 is the master regulator of centrosome duplication and its deficiency has recently been associated with Seckel syndrome (53). However, the precise role of PLK4 in genomic stability and the DNA damage response is still unclear. According to Kalay et al. $(32,112)$, mutated PLK4-Seckel fibroblasts obtained from patients revealed a G2/M delay, prolonged cell doubling time, impaired centriole biogenesis, and a disrupted mitotic morphology.

One plausible explanation of how different genetic deletions of centrosomal components in Seckel syndrome can lead to a similar outcome as the one caused by a mutation in ATR/ATRIP would be that centrosomes might contribute to ATR signaling. Moreover, $\mathrm{CHK} 1$, the downstream kinase of ATR, is associated with centrosomes, yet we could not detect the association of CHK1 and centrosomes, and this could be due to numerous cross-reactions. Instead, our results strongly suggest that impaired centrosome composition triggers the translocation of MLK3, followed by activation of $\mathrm{p38}$, MK2, and JUN. This signaling cascade induces replication stress, much like the deletion of ATR. These similar outcomes explain why the disruption of ATR signaling or centrosome integrity by genetic alterations can lead to very similar clinical conditions. It is conceivable to think that inhibiting or interfering with one or more partners of the activated stress pathway described in this thesis (MLK3-P38-MK2-JUN) might be beneficial for the treatment of patients with Seckel syndrome. For that purpose, we decided to evaluate the inhibition of MK2 in Seckel patient's cells. Although such patients are found rarely, this perspective remains to be assessed. As predicted, we managed to observe an improvement in DNA replication and cell proliferation in these cells after applying the MK2 inhibitor, and we also detected a lower level of $\mathrm{\gamma H} 2 \mathrm{AX}$, which is a marker for DNA damage. 


\section{DISCUSSION}

\subsection{Concluding remarks and future perspectives}

In this thesis, we described strategies for targeting several centrosomal components using small-molecule inhibitors of PLK4, a centrosomal protein required for centrosome duplication, or using siRNAs targeting CEP152, SASS6, among others. We observed that PLK4 inhibition alone was sufficient to impair DNA replication and cause DNA damage independently of mitosis. We also reported that centrosomes, the microtubule organizing center of the eukaryotic cell, and the mitotic spindle support DNA replication by controlling the MLK3-p38-MK2 signaling cascade and suppress the formation of RNA:DNA hybrids (R-loops). This work establishes a mechanistic link between the duplications of two significant components that need to be replicated once every cell cycle, i. e. the cellular genome and the centrosomes.

To put this study in perspective, we also found that inhibiting the function of centrosomal proteins in several cell lines affected their ability to replicate their DNA faithfully. We also observed a significant decrease in cell proliferation upon centrosomal depletion in these cell lines.

In this work, we further increased our understanding of the role of centrosome duplication in cancer cells, and for the first time, we revealed a mutual regulation between two main processes happening in our cells - DNA replication and centrosome duplication. We showed that centrosome integrity during $S$ phase is an essential factor for accurate DNA replication. Having a clear understanding of the pathways regulating centrosome integrity is of critical importance for therapeutic benefits.

For the past decades, there has been an increasing interest in understanding the mechanism that controls centriole duplication. The deeper understanding of the biology behind centrosome biogenesis will have significant ramifications to the development of therapeutic drugs. Developing drugs to target core components of the centrosome started with CFI-400945, which made it successfully to clinical trial phase II. CFI-400945 was firstly described as a potent PLK4 inhibitor, yet later on, it was revealed that it could also target other centrosomal components, such as Aurora B (82). Following that, the discovery of Centrinone B took place. Centrinone B is considered to be a more specific PLK4 inhibitor, and even though this drug still has not made it to the clinical trials, it managed to show promising results in in vitro studies (108). Despite that, Centrinone B only managed to reduce cell proliferation and in our case, induce replicative stress by generating R-loops without any signs of causing cell death, which could be used as a positive quality of this inhibitor. Centrinone $B$ alone cannot serve as an effective cancer drug, but rather it 


\section{DISCUSSION}

can be used to control cell proliferation, metastasis, and invasiveness through the regulation of actin cytoskeleton, as recently discussed by other researchers (152). More centrosomal components also need to be investigated as therapeutic targets to achieve centrosome depletion, such as CDK2, which is also implicated in DNA replication and centrosome duplication (10, 11, and 98) or PLK1 / Separase, which are required for licensing centriole duplication and controlling the number of newly generated centrioles $(119,130)$.

Currently, we understand more about how these pathways are being activated upon centrosome impairment, but what needs to be further investigated is how these pathways function in vivo. We also lack animal models that mimick the phenotype caused by centrosome dysfunction, and it would be a critical achievement to generate such models. Having animal models mimicking centrosome dysfunctions would indeed increase our current understanding of human diseases.

An alternative approach to target centrosomes in cancer is by developing drugs that are able to suppress centrosome clustering. Cancer cells with centrosome amplification tend to cluster them to ensure bipolar spindle assembly, while healthy cells do not require this. Inhibiting centrosome clustering in cancer cells is expected to have a lethal outcome due to the multipolar division and therefore this could provide a promising treatment option for tumors with amplified centrosomes. CW069 (153) and CCB02 (154) are two compounds, which have attracted the attention of the scientific community recently. CW069 inhibits the microtubule motor protein HSET, which is crucial for centrosome clustering in cancer cells. Inhibition of HSET was reported to reduce centrosome clustering and cell growth in cancer cells, without affecting healthy cells. CCB02, a different compound, inhibits the interaction between centrosomal-P4.1-associated protein (CPAP) and tubulin, thereby enhancing microtubule nucleation and inhibiting the following centrosome clustering.

Lastly, instead of targeting centrosomal components directly, one could target proteins that control the response to abnormalities in centrosome duplication. Since we identified a new centrosomal component that plays a significant role in sensing centrosome abnormalities, MLK3/MK2, might be appropriate targets in cancer. In addition to that, USP28 (79), another protein that has been associated with the mitotic surveillance pathway, could also be targeted in microcephaly patients, where this pathway is activated. 


\section{REFERENCES}

\section{REFERENCES}

1. Juliette Azimzadeh, a.M.B. Structure, and duplication of the centrosome. Cell Science 1202139 $2142(2007)$

2. Juliette Azimzadeh, a.M.B. Exploring the evolutionary history of centrosomes. Phil.Trans. R. Soc. B 369, (2014).

3. Maderspacher, F. Theodor Boveri, and the natural experiment. Current Biology 18, R279-R286 (2008).

4. Pihan, G.A. Centrosome dysfunction contributes to chromosome instability, chromoanagenesis, and genome reprogramming in cancer. Front Oncol 3, 277 (2013).

5. Nigg, E.A. \& Raff, J.W. Centrioles, centrosomes, and cilia in health and disease. Cell 139, 663-78 (2009).

6. Conduit, P.T., Wainman, A. \& Raff, J.W. Centrosome function and assembly in animal cells. Nat Rev Mol Cell Biol 16, 611-24 (2015).

7. Nam, H.J., Naylor, R.M. \& van Deursen, J.M. Centrosome dynamics as a source of chromosomal instability. Trends Cell Biol 25, 65-73 (2015).

8. Chavali, P.L., Putz, M. \& Gergely, F. Small organelle, big responsibility: the role of centrosomes in development and disease. Philos Trans R Soc Lond B Biol Sci 369(2014).

9. Mary K Harrison, A.M.A., Harold I Saavedra. The G1 phase Cdks regulate the centrosome cycle and mediate oncogene-dependent centrosome amplification. Cell Division 6, 2 (2011).

10. Onur Cizmecioglu, M.A., Ramona Bahtz, Florian Settele, Lena Ehret, Uta Haselmann-Weiß, Claude Antony, Ingrid Hoffmann. Cep152 acts as a scaffold for recruitment of Plk4 and CPAP to the centrosome. Journal of Cell Biology 191, 731-739 (2010).

11. Katharina F. Sonnen, A.-M.G., Eduard Anselm, York-Dieter Stierhof, Erich A. Nigg. Human Cep192 and Cep152 cooperate in Plk4 recruitment and centriole duplication. Journal of Cell Science 126, 3223-3233 (2013).

12. Arquint C, N.E. The PLK4-STIL-SAS-6 module at the core of centriole duplication. Biochem Soc Trans. 15, 1253-1263. (2016).

13. Vulprecht J, D.A., Tibelius A, Castiel A, Konotop G, Liu F, Bestvater F, Raab MS, Zentgraf H, Izraeli S, Krämer A. STIL is required for centriole duplication in human cells. J Cell Sci. 125, 1353-62 (2012).

14. Chii Shyang Fong, M.K., T. Tony Yang, Jung-Chi Liao, Meng-Fu Bryan Tsou. SAS-6 Assembly Templated by the Lumen of Cartwheel-less Centrioles Precedes Centriole Duplication. Developmental Cell 30, P238-245, (2014).

15. Keller D, O.M., Olivier N, Wachsmuth M, Mahen R, Wyss R, Hachet V, Ellenberg J, Manley S, Gönczy P. Mechanisms of HsSAS-6 assembly promoting centriole formation in human cells. J Cell Biol. 3, 697-712 (2014).

16. Schmidt TI, K.-S.J., Westendorf J, Le Clech M, Lavoie SB, Stierhof YD, Nigg EA. Control of centriole length by CPAP and CP110. Curr Biol. 19, 1005-11 (2009). 


\section{REFERENCES}

17. Tibelius A, M.J., Zentgraf H, Heilig CE, Neitzel H, Ducommun B, Rauch A, Ho AD, Bartek J, Krämer A. Microcephalin and pericentrin regulate mitotic entry via centrosome-associated Chk1. J Cell Biol. 185, 1149-57 (2009).

18. Lingle WL, B.S., Negron VC, D'Assoro AB, Boeneman K, Liu W, Whitehead CM, Reynolds C, Salisbury JL. Centrosome amplification drives chromosomal instability in breast tumor development. PNAS 19, 1978-83 ( 2002).

19. Renata Basto, K.B., Tatiana Vinadogrova, Nina Peel, Anna Franz,Alexey Khodjakov, JordanW.Raff. Centrosome Amplification Can Initiate Tumorigenesis in Flies. Cell 133, 1032-1042 ( 2008 ).

20. Chan, J.Y. A Clinical Overview of Centrosome Amplification in Human Cancers. Int J Biol Sci 7, 1122-1144 (2011).

21. Denu RA, Z.L., Kanugh C, Laffin J, Weaver BA, Burkard ME. Centrosome amplification induces high grade features and is prognostic of worse outcomes in breast cancer. BMC Cancer 29(2016).

22. Pihan GA, P.A., Wallace J, Knecht H, Woda B, Quesenberry P, Doxsey SJ. Centrosome defects and genetic instability in malignant tumors. Cancer Res 58, 3974-85 (1998).

23. Lingle WL, L.W., Ingle JN, Maihle NJ, Salisbury JL. Centrosome hypertrophy in human breast tumors: implications for genomic stability and cell polarity. PNAS 95, 2950-5 (1998).

24. Ghadimi BM, S.D., Difilippantonio MJ, Schröck E, Neumann T, Jauho A, Auer G, Ried T. Centrosome amplification and instability occurs exclusively in aneuploid, but not in diploid colorectal cancer cell lines, and correlates with numerical chromosomal aberrations. Genes Chromosomes Cancer 27, 183-90 (2000)

25. Godinho SA, P.R., Burute M, Dagher R, Su Y, Leung CT, Polyak K, Brugge JS, Théry M, Pellman D. Oncogene-like induction of cellular invasion from centrosome amplification. Nature 510, 167-71 (2014).

26. Habedanck R, S.Y., Wilkinson CJ, Nigg EA. The Polo kinase Plk4 functions in centriole duplication. Nat Cell Biol 7, 1140-6 (2005).

27. Tang CJ, L.S., Hsu WB, Lin YN, Wu CT, Lin YC, Chang CW, Wu KS, Tang TK. The human microcephaly protein STIL interacts with CPAP and is required for procentriole formation. EMBO J 30, 4790-804 (2011).

28. Loncarek , H.P., Magidson V, Khodjakov A. Control of daughter centriole formation by the pericentriolar material. Nat Cell Biol 10, 322-8 (2008).

29. Kushner EJ, F.L., Liu JY, Durrant JR, Rogers SL, Dudley AC, Bautch VL. Excess centrosomes disrupt endothelial cell migration via centrosome scattering. J Cell Biol 206, 257-72 (2014).

30. Marina M, S.H. Nek2 and Plk4: prognostic markers, drivers of breast tumorigenesis, and drug resistance. Front Biosci 19, 352-65 (2014).

31. Ganem NJ, G.S., Pellman D. A mechanism linking extra centrosomes to chromosomal instability. Nature 460, 278-82 (2009).

32. Kalay, E. et al. CEP152 is a genome maintenance protein disrupted in Seckel syndrome. Nat Genet 43, 23-6 (2011).

33. Gabrielle Wheway, L.N., and John T. Hancock. Signaling through the Primary Cilium. Front Cell Dev Biol, 8 (2016).

34. Annouck Luyten, X.S., Sarah Gondela, Ying Chen, Santiago Rompani, Ayumi Takakura, and Jing Zhoucorresponding author. Aberrant Regulation of Planar Cell Polarity in Polycystic Kidney Disease. J Am Soc Nephrol 21, 1521-1532 (2010). 


\section{REFERENCES}

35. Douglas Hanahan, R.A.W. The Hallmarks of Cancer. Cell 100, 57-70 (2000).

36. Guillaume Normand, R.W.K. Understanding Cytokinesis Failure. Adv Exp Med Biol 676, 27-55 (2011).

37. Erik Boye, A.L.-O., Kirsten Skarstad. Limiting DNA replication to once and only once. EMBO Rep 15, 479-483 (2000).

38. Dobbelstein, M. \& Sorensen, C.S. Exploiting replicative stress to treat cancer. Nat Rev Drug Discov 14, 405-23 (2015).

39. Camille Gelot, I.M., Bernard S. Lopez Replication Stress in Mammalian Cells and Its Consequences for Mitosis. Genes 6, 267-298 (2015).

40. Parker MW, B.M., Berger JM. Mechanisms and regulation of DNA replication initiation in eukaryotes. Crit Rev Biochem Mol Biol. 52, 107-144 (2017).

41. Barnum KJ, O.C.M. Cell cycle regulation by checkpoints. Methods Mol Biol., 1170-40 (2014).

42. Gaillard H, G.-M.T., Aguilera A. Replication stress, and cancer. Nat Rev Cancer 15, 276-89 (2015).

43. Zeman MK, C.K. Causes and consequences of replication stress. Nat Cell Biol 16, 2-9 (2014).

44. Irina Bruck, D.L.K. Cdc45 Protein-Single-stranded DNA Interaction Is Important for Stalling the Helicase during Replication Stress. J Biol Chem 288, 7550-7563 (2013).

45. Jackson SP, B.J. The DNA-damage response in human biology and disease. Nature 461, 1071-8 (2009).

46. Brown EJ, B.D. ATR disruption leads to chromosomal fragmentation and early embryonic lethality. Genes Dev 14, 397-402 (2000).

47. O'Driscoll, M., Ruiz-Perez, V.L., Woods, C.G., Jeggo, P.A. \& Goodship, J.A. A splicing mutation affecting expression of ataxia-telangiectasia and Rad3-related protein (ATR) results in Seckel syndrome. Nat Genet 33, 497-501 (2003).

48. Ogi, T. et al. Identification of the first ATRIP-deficient patient and novel mutations in ATR define a clinical spectrum for ATR-ATRIP Seckel Syndrome. PLoS Genet 8, e1002945 (2012).

49. Matilde Murga, S.B., Maria F. Montaña, Rebeca Soria, Francisca Mulero, Marta Cañamero, Youngsoo Lee, Peter J. McKinnon, Andre Nussenzweig, and Oscar Fernandez-Capetillo. A mouse model of the ATR-Seckel Syndrome reveals that replicative stress during embryogenesis limits mammalian lifespan. Nat Genet 41, 891-898 (2009).

50. Verloes A, D.S., Gressens P, Passemard S. Primary Autosomal Recessive Microcephalies and Seckel Syndrome Spectrum Disorders. GeneReviews (1993)

51. Guernsey DL, J.H., Hussin J, Arnold M, Bouyakdan K, Perry S, Babineau-Sturk T, Beis J, Dumas N, Evans SC, Ferguson M, Matsuoka M, Macgillivray C, Nightingale M, Patry L, Rideout AL, Thomas A, Orr A, Hoffmann I, Michaud JL, Awadalla P, Meek DC, Ludman M, Samuels ME. Mutations in centrosomal protein CEP152 in primary microcephaly families linked to MCPH4. AJHG 87, 40-51 (2010).

52. Marjanovic, M. et al. CEP63 deficiency promotes p53-dependent microcephaly and reveals a role for the centrosome in meiotic recombination. Nat Commun 6, 7676 (2015).

53. Martin, C.A. et al. Mutations in PLK4, encoding a master regulator of centriole biogenesis, cause microcephaly, growth failure, and retinopathy. Nat Genet 46, 1283-1292 (2014).

54. Griffith, E. et al. Mutations in pericentrin cause Seckel syndrome with defective ATR-dependent DNA damage signaling. Nat Genet 40, 232-6 (2008). 


\section{REFERENCES}

55. Rauch A, T.C., Schindler D, Wick U, Crow YJ, Ekici AB, van Essen AJ, Goecke TO, AI-Gazali L, Chrzanowska KH, Zweier C, Brunner HG, Becker K, Curry CJ, Dallapiccola B, Devriendt K, Dörfler A, Kinning E, Megarbane A, Meinecke P, Semple RK, Spranger S, Toutain A, Trembath RC, Voss E, Wilson L, Hennekam R, de Zegher F, Dörr HG, Reis A. Mutations in the pericentrin (PCNT) gene cause primordial dwarfism. Science 319, 816-9 (2008).

56. Matteo Berti, A.V. Replication stress: getting back on track. Nat Struct Mol Bio/ 32, 103-109 (2016).

57. Diffley, S.A.H.a.J.F.X. DNA Replication and Oncogene-Induced Replicative Stress. Current Biology 24, 435-444 (2014).

58. Minocherhomji, S. et al. Replication stress activates DNA repair synthesis in mitosis. Nature 528, 286-90 (2015).

59. Kotsantis, P. et al. Increased global transcription activity as a mechanism of replication stress in cancer. Nat Commun 7, 13087 (2016).

60. Aguilera, A. \& Garcia-Muse, T. R loops: from transcription byproducts to threats to genome stability. Mol Cell 46, 115-24 (2012).

61. Soultanas, P. The replication-transcription conflict. Transcription 2, 140-144 (2011).

62. Kevin S. Lang, A.N.H., Christopher N. Merrikh, Mark Ragheb, Hannah Tabakh, Alex J. Pollock, Joshua J. Woodward, Julia E. Dreifus, and Houra Merrikh. Replication-Transcription Conflicts Generate R-Loops that Orchestrate Bacterial Stress Survival and Pathogenesis. Cell 170, 787-799 (2017).

63. Wu, Y. Unwinding and Rewinding: Double Faces of Helicase? J Nucleic Acids 140601(2012).

64. García-Muse T, A.A. Transcription-replication conflicts: how they occur and how they are resolved. Nat Rev Mol Cell Biol 17, 553-63 (2016).

65. Mirkin EV, M.S. Mechanisms of transcription-replication collisions in bacteria. Mol Cell Bio/25, 88895 (2005).

66. Westover KD, B.D., Kornberg RD. Structural basis of transcription: separation of RNA from DNA by RNA polymerase II. Science 303, 1014-6 (2004).

67. Gan W, G.Z., Liu J, Gui T, Shen K, Manley JL, Li X. R-loop-mediated genomic instability is caused by impairment of replication fork progression. Genes Dev 25, 2041-56 (2011).

68. Lin YL, P.P. Interference between DNA replication and transcription as a cause of genomic instability. Curr Genomics 13, 65-73 (2012).

69. Jane Bayani, J.P., Joan Murphy, Barry Rosen, Maria Zielenska, and Jeremy A Squire. Distinct Patterns of Structural and Numerical Chromosomal Instability Characterize Sporadic Ovarian Cancer. Neoplasia 10, 1057-1065 (2008).

70. Natalia Vargas-Rondón, V.E.V., and Milena Rondón-Lagos. The Role of Chromosomal Instability in Cancer and Therapeutic Responses. Cancers (Basel) 10, 4 (2018).

71. Zeman MK, C.K. Causes and consequences of replication stress. Nat Cell Biol 16, 2-9 (2014).

72. Diffley, S.A.H.a.J.F.X. DNA Replication and Oncogene-Induced Replicative Stress. Current Biology 24, 435-444 (2014).

73. Marthiens V, R.M., Pennetier C, Tessier S, Paul-Gilloteaux P, Basto R. Centrosome amplification causes microcephaly. Nat Cell Biol. 15, 731-40 (2013).

74. Antonczak, A.K. et al. Opposing effects of pericentrin and microcephalin on the pericentriolar material regulate CHK1 activation in the DNA damage response. Oncogene 35, 2003-10 (2016). 


\section{REFERENCES}

75. Wang, C.Y., Huang, E.Y., Huang, S.C. \& Chung, B.C. DNA-PK/Chk2 induces centrosome amplification during prolonged replication stress. Oncogene 34, 1263-9 (2015).

76. Antonczak, A.K. et al. Opposing effects of pericentrin and microcephalin on the pericentriolar material regulate CHK1 activation in the DNA damage response. Oncogene 35, 2003-10 (2016).

77. Petrinac S, G.M., Bonni S, Nantais J, Hudson JW. Polo-like kinase 4 phosphorylates Chk2. Cell Cycle 8, 327-9 (2009).

78. Klusmann, I. et al. p53 Activity Results in DNA Replication Fork Processivity. Cell Rep 17, 18451857 (2016).

79. Lambrus, B.G. et al. A USP28-53BP1-p53-p21 signaling axis arrests growth after centrosome loss or prolonged mitosis. J Cell Biol 214, 143-53 (2016)

80. Shinmura K, B.R., Tarapore P, Fukasawa K. Direct evidence for the role of centrosomally localized p53 in the regulation of centrosome duplication. Oncogene 26, 2939-44 (2007).

81. Mason, J.M. et al. Functional characterization of CFI-400945, a Polo-like kinase 4 inhibitor, as a potential anticancer agent. Cancer Cell 26, 163-76 (2014).

82. Kawakami M, M.L., Zheng L, Chen Y, Rodriguez-Canales J, Mino B, Kurie JM, Roszik J, Villalobos PA, Thu KL, Silvester J, Cescon DW, Wistuba II, Mak TW, Liu X, Dmitrovsky E. Polo-like kinase 4 inhibition produces polyploidy and apoptotic death of lung cancers. PNAS 115, 1913-1918 (2018).

83. Anders W. Bailey, A.S., Pauline M. Chou, Tatiana Pundy, Samantha Gadd,Stacey L. Raimondi, Tadanori Tomita, and Simone Treiger Sredni. Polo-Like Kinase 4 (PLK4) Is Overexpressed in Central Nervous System Neuroblastoma (CNS-NB). Bioengineering 5, 96, (2018).

84. Hinchcliffe EH, M.F., Cham M, Khodjakov A, Sluder G. Requirement of a centrosomal activity for cell cycle progression through G1 into S phase. Science 291, 1547-50 (2001).

85. J, P. Four-dimensional control of the cell cycle. Nat Cell Biol 1, 73-9 (1999).

86. E. Bailly, J.P., T. Hunter, M. Bornens. Cytoplasmic accumulation of cyclin B1 in human cells: association with a detergent-resistant compartment and with the centrosome. Journal of Cell Science 101, 529-545 (1992).

87. Kathleen R. Lacey, P.K.J., Tim Stearns. Cyclin-dependent kinase control of centrosome duplication. pnas 96, 2817-2822 (2002).

88. Henderika M.J. Hut, W.L., Engbert H. Blaauw, Gert W.A. van Cappellen, Harm H. Kampinga, Ody C.M. Sibon. Centrosomes Split in the Presence of Impaired DNA Integrity during Mitosis. Mol Biol Cell 14, 1993-2004 (2003).

89. Yosuke Ichijima et al. DNA Lesions Induced by Replication Stress Trigger Mitotic Aberration and Tetraploidy Development. POLS (2010).

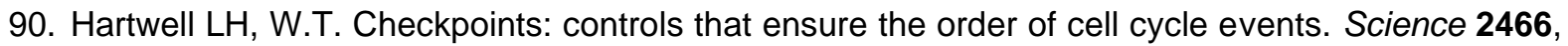
29-34 (1989).

91. Paraskevi Giannakakou, D.L.S., Yvona Ward, Kevin R. Webster, Mikhail V. Blagosklonny \&, T.F. p53 is associated with cellular microtubules and is transported to the nucleus by dynein. NATURE CELL BIOLOGY 2(2000).

92. Hsu LC, W.R. BRCA1 is associated with the centrosome during mitosis. PNAS 95, 12983-8 (1998).

93. Tarapore P, F.K. Loss of p53 and centrosome hyper amplification. Oncogene 21, 6234-40 (2002).

94. Ouyang X, W.X., Xu K, Jin DY, Cheung AL, Tsao SW, Wong YC. Effect of p53 on centrosome amplification in prostate cancer cells. Biochim Biophys Acta 1541, 212-20 (2001). 


\section{REFERENCES}

95. K Hanashiro, M.K., Y Geng, P Sicinski, K Fukasawa. Roles of cyclins A and E in induction of centrosome amplification in p53-compromised cells. Oncogene 27, 5288-5302 (2008).

96. el-Deiry WS, T.T., Velculescu VE, Levy DB, Parsons R, Trent JM, Lin D, Mercer WE, Kinzler KW, Vogelstein B. WAF1, a potential mediator of p53 tumor suppression. Cell 75, 817-25 (1993).

97. Hollander MC, F.A.J. Genomic instability, centrosome amplification, cell cycle checkpoints, and Gadd45a. Oncogene 21, 6228-33 (2002).

98. Rebecca L. Ferguson, J.L.M. Centrosomal Localization of Cyclin E-Cdk2 is Required for Initiation of DNA Synthesis. Curr Biol. 20, 856-860 (2010).

99. Antonczak, A.K. et al. Opposing effects of pericentrin and microcephalin on the pericentriolar material regulate CHK1 activation in the DNA damage response. Oncogene 35, 2003-10 (2016).

100. L A Tibbles, Y.L.I., F Kiefer, J Chan, $N$ Iscove, J R Woodgett, and N J Lassam. MLK-3 activates the SAPK/JNK and p38/RK pathways via SEK1 and MKK3/6. The EMBO Journal 15, 7026-7035 (1996).

101. Lambert JM, K.A., Graves LM, Campbell SL, Der CJ. Role of MLK3-mediated activation of p70 S6 kinase in Rac1 transformation. J Biol Chem. 277, (2002).

102. Dipika Gupta, B.L., Ann Cowan, and Christopher D. Heinen. ATR-Chk1 activation mitigates replication stress caused by mismatch repair-dependent processing of DNA damage. PNAS 115 1523-1528 (2018).

103. Breitwieser, W. et al. Feedback regulation of p38 activity via ATF2 is essential for survival of embryonic liver cells. Genes Dev 21, 2069-82 (2007).

104. Klusmann, I. et al. Chromatin modifiers Mdm2 and RNF2 prevent RNA: DNA hybrids that impair DNA replication. Proc Natl Acad Sci U S A 115, E11311-E11320 (2018).

105. Beli P, L.N., Wagner SA, Weinert BT, Olsen JV, Baskcomb L, Mann M, Jackson SP, Choudhary C. Proteomic investigations reveal a role for RNA processing factor THRAP3 in the DNA damage response. Mol Cell 46, 212-25 (2012).

106. Li, H.-C.C.X.Z.J.L.X.Z.J.C.H.T.-H.W.I.J.A.B.Y.H.R. BRCA1-associated R-loop affects transcription and differentiation in breast luminal epithelial cells. Nucleic Acids Research 47, 50865099 (2019).

107. Storchová, N.K.C.Z. The diverse consequences of aneuploidy. NATURE CELL BIOLOGY21, 5462 (2019).

108. Wong, Y.L. et al. Cell biology. Reversible centriole depletion with an inhibitor of Polo-like kinase 4. Science 348, 1155-60 (2015).

109. Yigit, G. et al. Mutations in CDK5RAP2 cause Seckel syndrome. Mol Genet Genomic Med 3, 46780 (2015).

110. D'Andrea, A.D. BRCA1: A Missing Link in the Fanconi Anemia/BRCA Pathway. Cancer Discov 3, 376-378 (2013).

111. Levine, M.S. et al. Centrosome Amplification Is Sufficient to Promote Spontaneous

Tumorigenesis in Mammals. Dev Cell 40, 313-322 e5 (2017).

112. Tuba Dinçer, G.Y.-B., Akgün Ölmez, İdris Er, Yavuz Dodurga, Özmert MA Özdemir, Bayram Toraman, Adem Yıldırım, Nuran Sabir, Nurten A Akarsu, C Nur Semerci, and Ersan Kalay,. Analysis of centrosome and DNA damage response in PLK4 associated Seckel syndrome. European Journal of Human Genetics 25, 1118-1125 (2017). 


\section{REFERENCES}

113. Passerini, V. et al. The presence of extra chromosomes leads to genomic instability. Nat Commun7,10754(2016).

114. Kopper, F. et al. Damage-induced DNA replication stalling relies on MAPK-activated protein kinase 2 activity. Proc Natl Acad Sci U S A 110, 16856-61 (2013).

115. Zhou, F., Xu, Y. \& Hou, X.Y. MLK3-MKK3/6-P38MAPK cascades following N-methyl-D-aspartate receptor activation contributes to amyloid-beta peptide-induced apoptosis in SH-SY5Y cells. $J$ Neurosci Res 92, 808-17 (2014).

116. Swenson, K.I., Winkler, K.E. \& Means, A.R. A new identity for MLK3 as an NIMA-related, cell cycle-regulated kinase that is localized near centrosomes and influences microtubule organization. Mol Biol Cell 14, 156-72 (2003).

117. Chadee, D.N. et al. Mixed-lineage kinase 3 regulates B-Raf through maintenance of the BRaf/Raf-1 complex and inhibition by the NF2 tumor suppressor protein. Proc Natl Acad Sci U S A 103, 4463-8 (2006).

118. Jorg Hartkamp, J.T., and Ulf R. Rapp. The JNK/SAPK Activator Mixed Lineage Kinase 3 (MLK3) Transforms NIH 3T3 Cells in a MEK-dependent Fashion1. CANCER RESEARCH (1999).

119. Shen, M. et al. Centrosomal protein FOR20 is essential for S-phase progression by recruiting Plk1 to centrosomes. Cell Res 23, 1284-95 (2013).

120. Denisenko TV, S.I., Gogvadze V, Zhivotovsky B. Mitotic catastrophe and cancer drug resistance: A link that must to be broken. Drug Resist Updat. 24, 1-12 (2016).

121. Meraldi, P. Centrosomes in spindle organization and chromosome segregation: a mechanistic view. Chromosome Res 24, 19-34 (2016).

122. O'Leary, B., Finn, R.S. \& Turner, N.C. Treating cancer with selective CDK4/6 inhibitors. Nat Rev Clin Oncol 13, 417-30 (2016).

123. Whitfield, M.L. et al. Identification of genes periodically expressed in the human cell cycle and their expression in tumors. Mol Biol Cell 13, 1977-2000 (2002).

124. Kopper, F., Binkowski, A.M., Bierwirth, C. \& Dobbelstein, M. The MAPK-activated protein kinase 2 mediates gemcitabine sensitivity in pancreatic cancer cells. Cell Cycle 13, 884-9 (2014).

125. Zheng, C. et al. MAPK-activated protein kinase-2 (MK2)-mediated formation and phosphorylationregulated dissociation of the signal complex consisting of p38, MK2, Akt, and Hsp27. J Biol Chem 281, 37215-26 (2006).

126. Mikule K, D.B., Kaldis $P$, Jurcyzk A, Hergert $P$, Doxsey $S$. Loss of centrosome integrity induces p38-p53-p21-dependent G1-S arrest. Nat Cell Biol. 9, (2007).

127. Srsen V, G.N., Dammermann A, Merdes A. Inhibition of centrosome protein assembly leads to p53-dependent exit from the cell cycle. J Cell Biol. 174, 625-30 (2006).

128. Anderson, D.R., Meyers, M. J., Vernier, W. F., Mahoney, M. W., Kurumbail, R. G., Caspers, N., Poda, G. I., Schindler, J. F., Reitz, D. B., and Mourey, R. J. Pyrrolopyridine inhibitors of mitogenactivated protein kinase-activated protein kinase 2 (MK-2), J Med Chem . 50, 2647-2654. (2007).

129. Tollenaere, M.A. et al. p38- and MK2-dependent signalling promotes stress-induced centriolar satellite remodelling via 14-3-3-dependent sequestration of CEP131/AZI1. Nat Commun 6, 10075 (2015).

130. Vertii A, I.M., Zimmerman W, Hehnly H, Kant S, Doxsey S. The Centrosome Undergoes Plk1Independent Interphase Maturation during Inflammation and Mediates Cytokine Release. Dev Cell 37, 377-386 (2016). 


\section{REFERENCES}

131. Tsekrekou, M., Stratigi, K. \& Chatzinikolaou, G. The Nucleolus: In Genome Maintenance and Repair. Int J Mol Sci 18 (2017).

132. Lindstrom, M.S. et al. Nucleolus as an emerging hub in maintenance of genome stability and cancer pathogenesis. Oncogene 37, 2351-2366 (2018).

133. O'Sullivan, J.M., Pai, D.A., Cridge, A.G., Engelke, D.R. \& Ganley, A.R. The nucleolus: a raft adrift in the nuclear sea or the keystone in nuclear structure? Biomol Concepts 4, 277-86 (2013).

134. Schofer, C. \& Weipoltshammer, K. Nucleolus, and chromatin. Histochem Cell Biol 150, 209-225 (2018).

135. She QB, M.W., Dong Z. Role of MAP kinases in UVB-induced phosphorylation of p53 at serine 20. Oncogene 21, 1580-9 (2002).

136. Cimprich, S.H.a.K.A. Conflict resolution in the genome: how transcription and replication make it work. Cell 167, 1455-1467 (2016).

137. Chen Z, I.V., McManus M, Wang L, Dynlacht BD. CP110, a cell cycle-dependent CDK substrate, regulates centrosome duplication in human cells. Dev Cell 3, 339-50 (2002).

138. M, L.C. Role of CAP350 in centriolar tubule stability and centriole assembly. PLoS One. 3 (2008).

139. Arquint, C., Gabryjonczyk, A.M. \& Nigg, E.A. Centrosomes as signalling centres. Philos Trans R Soc Lond B Biol Sci 369 (2014).

140. Staples, C.J. et al. The centriolar satellite protein Cep131 is important for genome stability. J Cell Sci 125, 4770-9 (2012).

141. Tollenaere MA, M.N., Bekker-Jensen S. Centriolar satellites: key mediators of centrosome functions. Cell Mol Life Sci. 72, 11-23 (2015).

142. Bug, M. \& Dobbelstein, M. Anthracyclines induce the accumulation of mutant p53 through E2F1dependent and -independent mechanisms. Oncogene 30, 3612-24 (2011).

143. lovino F, L.L., Amato A, Di Leonardo A. RB acute loss induces centrosome amplification and aneuploidy in murine primary fibroblasts. Mol Cancer 20, 38 (2006).

144. Lentini L, I.F., Amato A, Di Leonardo A. Centrosome amplification induced by hydroxyurea leads to aneuploidy in pRB deficient human and mouse fibroblasts. Cancer Lett 238, 153-60 (2006).

145. Suhail TV, S.P., Manna TK. Suppression of centrosome protein TACC3 induces G1 arrest and cell death through activation of p38-p53-p21 stress signaling pathway. Eur J Cell Biol. 94, 90-100 (2015).

146. Cha, H., Dangi, S., Machamer, C.E. \& Shapiro, P. Inhibition of mixed-lineage kinase (MLK) activity during G2-phase disrupts microtubule formation and mitotic progression in HeLa cells. Cell Signal 18, 93-104 (2006).

147. Rangasamy V, M.R., Mehrotra S, Sondarva G, Ray RS, Rao A, Chatterjee M, Rana B, Rana A. Estrogen suppresses MLK3-mediated apoptosis sensitivity in ER+ breast cancer cells. Cancer Res 70, 1731-40 (2010).

148. Anindita Bhoumik, P.L.B., and Ze'ev Ronai. ATF2 on the Double - Activating Transcription Factor and DNA Damage Response Protein. Pigment Cell Res 20, 498-506 (2007).

149. Monje P, H.-L.J., Lyons RJ, Castellone MD, Gutkind JS. Regulation of the transcriptional activity of c-Fos by ERK. A novel role for the prolyl isomerase PIN1. J Biol Chem 280, 35081-4 (2005).

150. Lau E, R.Z. ATF2 - at the crossroad of nuclear and cytosolic functions. J Cell Sci. 125, 2815-24 (2012). 


\section{REFERENCES}

151. Anindita Bhoumik, S.T., Wolfgang Breitweiser,Yosef Shiloh, Nic Jones, Ze'ev Ronai. ATMDependent Phosphorylation of ATF2 Is Required for the DNA Damage Response. Molecular Cell 18, 577-587 (2005).

152. Kazazian K, G.C., Wu H, Brashavitskaya O, Xu R, Dennis JW, Gingras AC, Swallow CJ. Plk4 Promotes Cancer Invasion and Metastasis through Arp2/3 Complex Regulation of the Actin Cytoskeleton. Cancer Res 77, 434-447 (2017).

153. Watts CA, R.F., Bender A, Bond PJ, Korb O, Kern O, Riddick M, Owen P, Myers RM, Raff J, Gergely F, Jodrell DI, Ley SV. Design, synthesis, and biological evaluation of an allosteric inhibitor of HSET that targets cancer cells with supernumerary centrosomes. Chem Biol. 20, 1399-410 (2013).

154. Mariappan A, S.K., Schorpp K, Zhao F, Minakar A, Zheng X, Mandad S, Macheleidt I, Ramani A, Kubelka T, Dawidowski M, Golfmann K, Wason A, Yang C, Simons J, Schmalz HG, Hyman AA, Aneja R, Ullrich R, Urlaub H, Odenthal M, Büttner R, Li H, Sattler M, Hadian K, Gopalakrishnan J. Inhibition of CPAP-tubulin interaction prevents proliferation of centrosome-amplified cancer cells. EMBO J 38(2019). 


\section{Supplementary Figure 1}

\section{MAP3K1 depleting prevents the activation of p38/MK2 in the H1299 cell line}

A

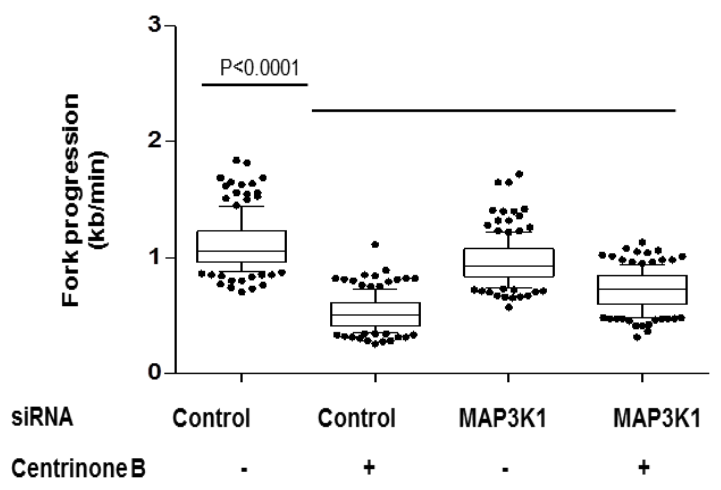

C

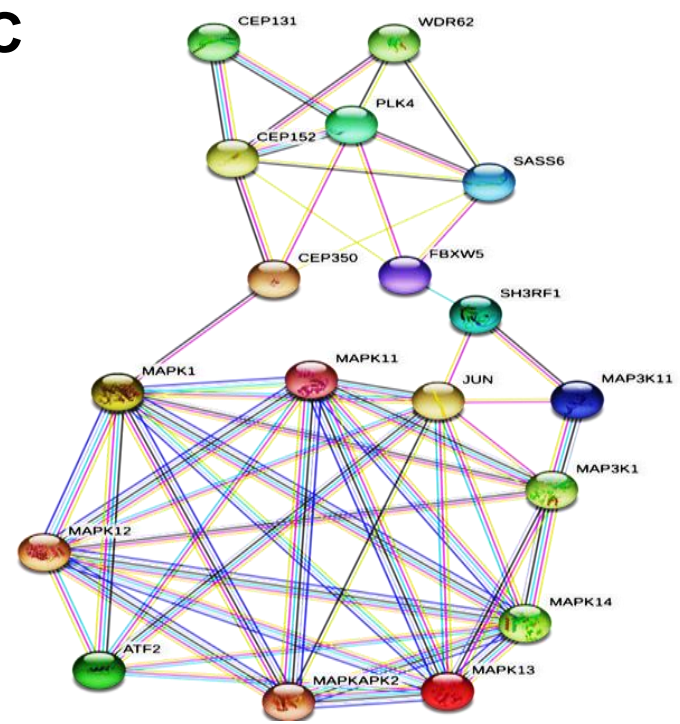

B

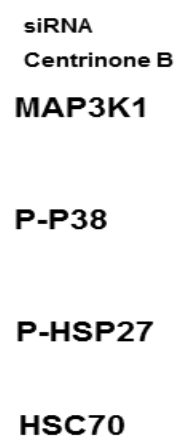

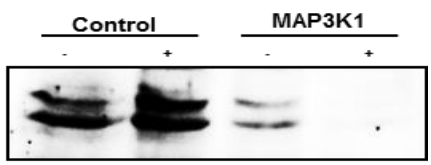
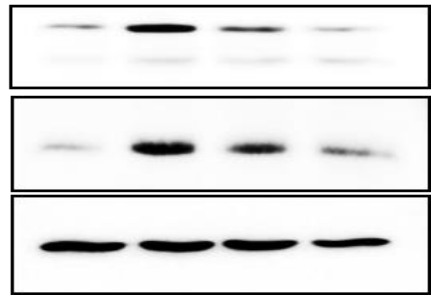

Figure supplementary 1: MAP3K1 depleting prevents the activation of p38/MK2 in the H1299 cell line.

(A) MAP3K1 depletion rescues DNA replication when centrosomal components are removed by pool siRNA. Synchronized H1299 cells were reverse transfected with $10 \mathrm{nM}$ siRNAs against the targets MAP3K1 for 72 hours in total and treated with $500 \mathrm{nM}$ Centrinone for $48 \mathrm{hrs}$ then subjected to fiber assays. 
(B) MAP3K1 knockdown diminishes p38/MK2 activation upon centrosome depletion. Upon transfection as in (A), pathway activation was detected by phospho-specific antibodies.

(C) protein-protein interaction using the STRING online tool. The STRING database contains information from numerous sources, including experimental data, computational prediction methods, and public text collections

Supplementary Table 1: Phosphoproteomics reveals a significant phosphorylation level of MAP3K1, CHK1, after Centrinone B treatment in H1299.

A

B

\begin{tabular}{|c|c|c|c|c|}
\hline $\begin{array}{c}\text { Positions } \\
\text { within } \\
\text { proteins }\end{array}$ & Gene names & Amino acid & $\begin{array}{c}\text { Ratio H/L } \\
\text { normalized 1 }\end{array}$ & $\begin{array}{c}\text { Ratio H/L } \\
\text { normalized 2 }\end{array}$ \\
\hline 275 & $\begin{array}{c}\text { MAP3K1 } \\
\text { (S275) }\end{array}$ & S & 4.2971 & 0.24878 \\
\hline 285 & $\begin{array}{c}\text { MAP3K1 } \\
\text { (T285) }\end{array}$ & T & 4.2971 & 0.24878 \\
\hline 292 & $\begin{array}{c}\text { MAP3K1 } \\
\text { (S292) }\end{array}$ & S & 3.8358 & 0.24605 \\
\hline 507 & $\begin{array}{c}\text { MAP3K1 } \\
\text { (S507) }\end{array}$ & S & 2.6874 & 0.28236 \\
\hline 170 & $\begin{array}{c}\text { SQSTM1 } \\
\text { (S170) }\end{array}$ & S & 3.2842 & 0.32253 \\
\hline 307 & $\begin{array}{c}\text { CHEK1 (S307) } \\
1068\end{array}$ & S & 1.504 & 0.71324 \\
\hline 1567 & MDC1 (T1567) & T & 1.9761 & 0.52428 \\
\hline 1664 & MDC1 (T1664) & T & 2.1806 & 0.59402 \\
\hline
\end{tabular}

\begin{tabular}{|l|c|c|}
\hline & \multicolumn{2}{|c|}{ log2 transform } \\
\hline Gene Name & Rep1 & Rep2 \\
\hline MAP3K1 (S275) & 2.10336335 & 2.00705759 \\
\hline MAP3K1 (T285) & 2.10336335 & 2.00705759 \\
\hline MAP3K1 (S292) & 1.9395275 & 2.02297658 \\
\hline MAP3K1 (S507) & 1.42621107 & 1.82439237 \\
\hline SQSTM1 (S170) & 1.71554199 & 1.63249474 \\
\hline CHEK1 (S307) & 0.58880457 & 0.48754048 \\
\hline MDC1 (S1068) & 0.69545938 & 1.06691086 \\
\hline MDC1 (T1567) & 0.98265596 & 0.93159058 \\
\hline MDC1 (T1664) & 1.12472515 & 0.75141659 \\
\hline
\end{tabular}

C

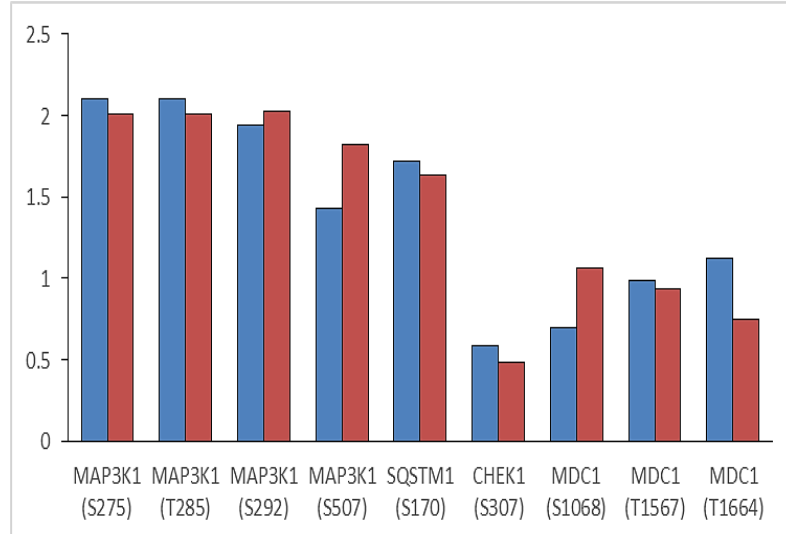




\section{SUPPLEMENTARY MATERIAL}

Phosphoproteomics reveals a significant phosphorylation level of MAP3K1, CHK1, after Centrinone B treatment in H1299.

(A) Table.1 shows the most unregulated proteins upon Centrinone B treatment. Labeled synchronized H1299 cells were treated with 500nM Centrinone B for 48hours then subjected to Masspec analysis.

(B) Table 2 shows the log2 of the most unregulated proteins upon Centrinone B treatment as in (A).

(C) The bar plot shows selected proteins associated with a DNA stress response that is significantly recruited to chromatin after Centrinone $B$ treatment. 


\section{Acknowledgment}

I want to thank Matthias Dobbelstein for his endless support and encouragement during my project. I am very grateful for having been allowed to establish and work on my project during my Ph.D. time. All along the way, Matthias has guided me scientifically, encouraged my personal development, and made my stay in the lab very successful and enjoyable.

I would also like to thank my thesis advisory committee members Dr. Bernd Wollnik and Dr. Steven Johnsen, for fruitful discussions and valuable input on my work during our thesis advisory committee meetings. Furthermore, I thank Dr. Roland Dosch, Prof. Dr. Heidi Hahn, Dr. Ufuk Günesdogan and Dr. Nuno Raimundo for assessing my work in the extended thesis committee.

My three years of stay in the Department of Molecular Oncology have been a great time in the company of many inspiring people. I thank all members of the Molecular Oncology, Tumour Epigenetics, and Molecular groups for creating a pleasant working environment, providing input during our joint seminars, and being supportive in all matters.

In particular, I would like to thank Karola Metze and Antje Dickmanns for giving me such a good start in the lab; my office crew with all past and present members for scientific discussions as well as fun times - Kim, Vella, Antonia, Luisa, Valentina, Celeste and Joe. I would also like to thank everyone that has contributed to this project, in particular, Kim, Antonia, and Vella, for their great help and contributions in completing this part of the project and taking it further in the future. Special thanks go to Kim, Vella, Celeste, Luisa, Valentina for proofreading my thesis.

I'm very grateful for Deutsche Forschungsgemeinschaft DFG (FOR 2800), to support and fund my work.

Last but not least, I would like to thank my family, friends, and especially my boyfriend (Gokhan) for being there for me during my up and down days. I am incredibly thankful for your understanding, patience, and encouragement during the last couple of years. 


\section{Abbreviations}

${ }^{\circ} \mathrm{C}$

APS

ATM

ATR

ATRIP

bp

BSA

CDK

cDNA

$\mathrm{CHK}$

CldU

$\mathrm{CO} 2$

Ct

ctrl

DDR

DMEM

DMSO
Degree Celsius

ammonium persulfate

ataxia-telangiectasia mutated

ATM- and Rad3-related

ATR interacting protein

base pair

bovine serum albumin

cyclin-dependent kinase

complementary DNA

checkpoint kinase

chlorodeoxyuridine

carbondioxide

cycle threshold

control

DNA damage response

Dulbecco's modified Eagle medium

Dimethylsulfoxide 


\begin{tabular}{ll} 
& \multicolumn{1}{c}{ ABBREVIATIONS } \\
\hline DNA & deoxyribonucleic acid \\
dNTP & deoxyribonucleotide triphosphate \\
DSB & double-strand break \\
dsDNA & double-strand DNA \\
EdU & 5-ethynyl-2'-deoxyuridine \\
EtOH & ethanol \\
FCS & fetal calf serum
\end{tabular}




\section{Zainab Tayeh}

Address Zeppelinstraße. 3

37083 Göttingen

Germany

Contact Telefon: +4917674456495

E-Mail: zainab tayeh@yahoo.com

E-Mail: zainab.tayeh@med.uni-

goettingen.de

Linkedln:

www.linkedin.com/in/zainab-tayeh-

$\underline{48394058 /}$

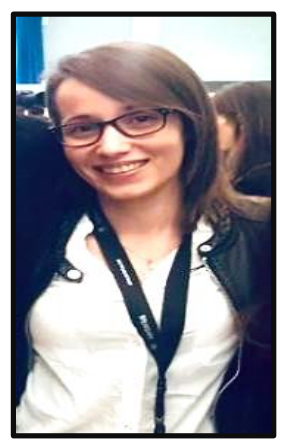

Born 22.03.1987

Kuwait, Kuwait

Nationality Jordanian

Education, Internship and Work Experience

2017-present

2015-2017

2013-2015

2013

2012-2013
Ph.D. Candidate at Göttingen Center of Molecular Biosciences, GZMB, Faculty of Medicine, University of Göttingen, Germany.

Internship at the molecular science and biochemistry department of Weizmann Institute of Science, Israel.

Master studies in the medicinal school of Ben Gurion University of Negev, Israel. The final grade (Cum Laude)

Internship at the Dead Sea \& Arava science center, Israel

Environmental Studies and peace leadership at Arava Institute, Israel. 
Telecommunication Promoters Manager, Jordan, Tanzania, UAE and Turkey

2011

Internship in the department of molecular oncology of Jordan University

2010-2011

Internship and training courses (hematology, flow cytometry, molecular labs, and HLA) at al Hussein center for cancer, Jordan

2009

Internship at the molecular biology lab, ALKHALED hospital, Jordan.

2005-2009

B.Sc. studies, Genetic Engineering and Biotechnology, Jordan University of Science and Technology

\section{Publications}

Jul 30, 2018

Asteriscus graveolens Extract in Combination with Cisplatin/Etoposide/Doxorubicin Suppresses Lymphoma Cell Growth through Induction of Caspase-3 Dependent Apoptosis International Journal of Molecular Sciences Tayeh Z, Ofir R.

https://www.ncbi.nlm.nih.gov/pubmed/30061495

Jul 24, 2018

Molecular Mode of Action of Asteriscus graveolens as an Anticancer Agent International Journal of Molecular Sciences Tayeh Z, Dudai N, Schechter A, Chalifa-Caspi V, Barak S, Ofir R.

https://www.ncbi.nlm.nih.gov/pubmed/30042356

Jun 29, 2019

Centrosome impairment causes DNA replication stress through MLK3/MK2 signaling and R-loop formation

Submitted to Cell Research

Zainab Tayeh, Kim Stegmann, Antonia Kleeberg, Mascha Friedrich, Bernd Wollnik, and Matthias Dobbelstein 


\section{Soft skills}

Languages

Software

Soft skills courses

Teaching and Supervision
Arabic (Mother's tongue), English (Advanced), German (A1).

KNIME software

Python (Part 1)

Python (Part 2)

MS office

DNA analysis tools

Image $\mathrm{J}$

Prism

Know yourself - emphasize your strengths.

Time management in doctoral research.

Scientific communication.

Good scientific practice for biologists.

Instruction of 4-day methods course for Masters students on 'DNA techniques' including DNA fiber assay, RNA isolation, cDNA synthesis, and quantitative RT PCR

Supervision of two masters students during their lab rotation

Supervision of two masters students during their master theses and one student for medical theses

Conducted interactive tutorials for Masters students 


\section{$\underline{\text { Technical skills }}$}

\section{Lab experiences}

Cell culture, primary cell culture, FACS, PCR, qPCR,

DNA fiber assay, Western blot,

Microscopic (light, fluorescent, and confocal), Cells transfection-transduction (plasmid overexpression, Knocked down, lentivirus and retrovirus )

Colony sequencing,

Chromatin fractionation, Dot blot analysis, R-loop detection, EdU staining Chromosomes spreading

\section{Social Engagement and Interest}

\section{Social Engagement}

Member of Youth Spirit Center in Jordan for non-formal educational, Jordan The youngest member in the Center. Contributed to educational and cultural seminars that provoke educational pursuit for the youth. Organized cultural events.

Y.M.C. A summer camp, Jordan Overseeing over 30 children, ages between 7 \& 10. Obtained first-aid training throughout the course. Carried out creative ways to entertain and educate the children.

\section{$\underline{\text { Referees }}$}

PhD

Prof. Dr. med. Matthias Dobbelstein, Institute of Molecular Oncology Göttingen Center of Molecular Biosciences, GZMB, Faculty of Medicine, University of Göttingen, Germany, mdobbel@unigoettingen.de

Rivka Ofir, Ph.D., 
Regenerative Medicine \& Stem Cell Research Center and the Dead Sea \& Arava Science Center, Ben Gurion University of the Negev, rivir@bgu.ac.il

Simon Barak, Prof.

M.Sc.

The Ben-Gurion University of the Negev, French Associates Institute for Agriculture and Biotechnology of Drylands, Jacob Blaustein Institutes for Desert Research, Midreshet BenGurion, Israel, simon@bgu.ac.il.

Internship

Dr. Abdullah Al-Abadi, Director of hematology and blood cancer department. The University of Jordan, Faculty of Medicine, admin@ju.edu.jo

Internship

Kais Al-Mousa, Ph.D., Director of Clinical Chemistry Laboratory, King Hussein Cancer Center, kmousa@khcc.jo 


\section{Affidavit}

Herewith I declare that the $\mathrm{PhD}$ Thesis entitled "Centrosome integrity as a determinant ofreplication stress" was written independently and with no other sources and aids than quoted.

Zainab Tayeh

Göttingen, November 2019 